\title{
Ambidexterity and Height
}

\author{
Shachar Carmeli* Tomer M. Schlank ${ }^{\dagger} \quad$ Lior Yanovski ${ }^{\ddagger}$
}

\begin{abstract}
We introduce and study the notion of semiadditive height for higher semiadditive $\infty$ categories, which generalizes the chromatic height. We show that the higher semiadditive structure trivializes above the height and prove a form of the redshift principle, in which categorification increases the height by one. In the stable setting, we show that a higher semiadditive $\infty$-category decomposes into a product according to height, and relate the notion of height to semisimplicity properties of local systems. We place the study of higher semiadditivity and stability in the general framework of smashing localizations of $\operatorname{Pr}^{L}$, which we call modes. Using this theory, we introduce and study the universal stable $\infty$-semiadditive $\infty$-category of semiadditive height $n$, and give sufficient conditions for a stable 1-semiadditive $\infty$-category to be $\infty$-semiadditive.
\end{abstract}

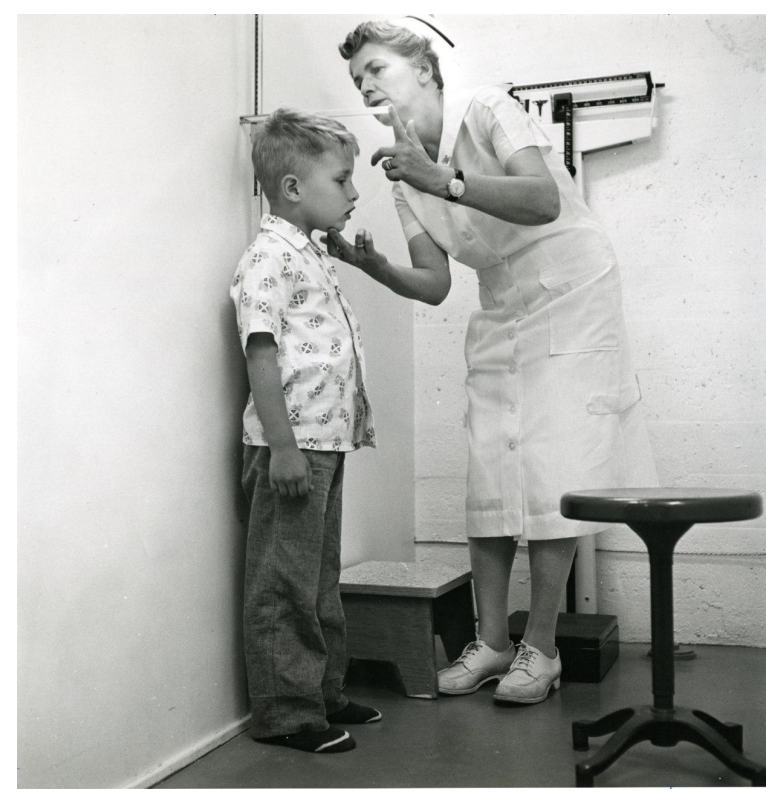

Nurse measures height of child for Tecumseh Health study, 1959, HS15323, Alumni Association (University of Michigan) records, UM News Service, Bentley Historical Library, University of Michigan.

* Department of Mathematics, Weizmann Institute of Science.

$\dagger$ Einstein Institute of Mathematics, Hebrew University of Jerusalem.

$\ddagger$ Max Planck Institute for Mathematics. 


\section{Contents}

1 Introduction $\quad 2$

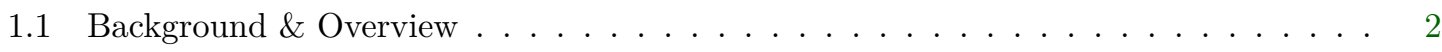

1.2 Main Results . . . . . . . . . . . . . . . . . . . . . . . 4

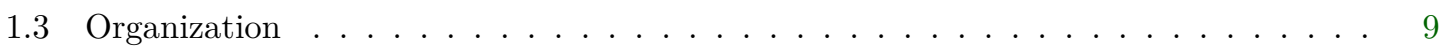

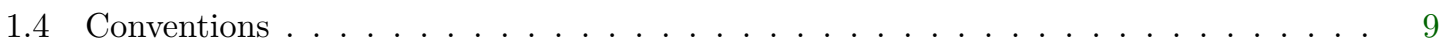

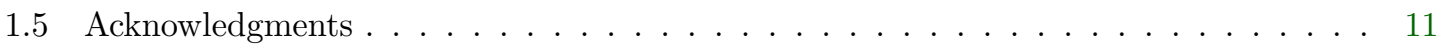

2 Semiadditivity 11

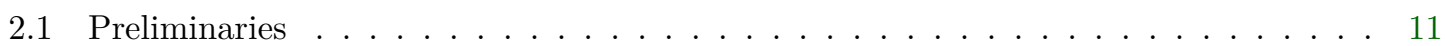

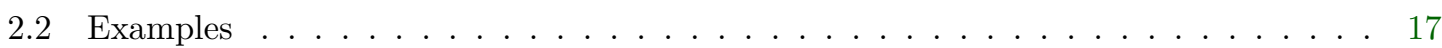

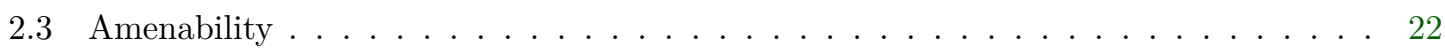

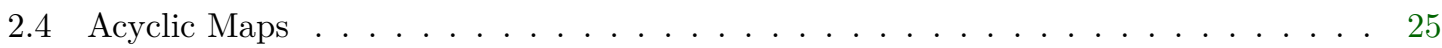

3 Height 29

3.1 Semiadditive Height . . . . . . . . . . . . . . . . . . . . . . . . . . . . . 29

3.2 Bounded Height . . . . . . . . . . . . . . . . . . . . . . . . . . . . . 33

3.3 Semiadditive Redshift . . . . . . . . . . . . . . . . . . . . . 36

4 Stability $\quad 38$

4.1 Recollement ............................. 39

4.2 Height Decomposition ........................... . . 44

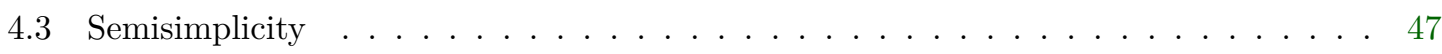

4.4 Chromatic Examples ........................... 49

5 Modes $\quad 51$

5.1 Idempotent Algebras ........................... 51

5.2 Theory of Modes . . . . . . . . . . . . . . . . . . . . . 56

5.3 Modes of Semiadditivity . . . . . . . . . . . . . . . . . . . 62

5.4 1-Semiadditive Decomposition . . . . . . . . . . . . . . . . . 66

5.5 Semiadditive vs. Stable Height . . . . . . . . . . . . . . . . . . . 70

$\begin{array}{lr}\text { References } & 76\end{array}$

\section{Introduction}

\subsection{Background \& Overview}

Chromatic homotopy theory springs from the deep and surprising connection between the $\infty$ category of spectra and the stack of formal groups. In particular, the height filtration on the 
latter is mirrored by the "chromatic height filtration" on the former. This connection begins with Quillen's work on the complex cobordism spectrum $M U$, showing that the ring $\pi_{*} M U$ carries the universal formal group law. Formal group laws admit a notion of ( $p$-typical) height for every prime $p$. This notion can be defined in terms of a certain sequence of classes $v_{n} \in \pi_{2\left(p^{n}-1\right)} M U$ as follows: If $v_{0}, \ldots, v_{n-1}$ vanish, then the height is $\geq n$, and if $v_{n}$ is invertible, then the height is $\leq n$. This algebraic filtration has a spectrum level manifestation in the form of the Morava $K$-theories $K(n)$, which are certain $M U$-algebras with the property $\pi_{*} K(n) \simeq \mathbb{F}_{p}\left[v_{n}^{ \pm 1}\right]$. This suggests that the $K(n)$-s are concentrated at height exactly $n$, and the corresponding Bousfield localizations $\operatorname{Sp}_{K(n)} \subseteq \operatorname{Sp}$ can then be considered as the "monochromatic layers" of the chromatic height filtration. The process of $K(n)$-localization can be loosely thought of as completion with respect to $v_{0}, \ldots, v_{n-1}$ followed by the inversion of $v_{n}$.

By the work of Hopkins, Devinatz, and Smith (see [HS98, Theorems 9 and 4.12]), the $v_{n}$-operations can be inductively lifted to finite spectra, without $M U$-module structure. More precisely, a finite $p$-local spectrum $F$ is said to have type $n$, if $n$ is the lowest integer for which the $K(n)$-localization of $F$ does not vanish. Given a type $n$ finite spectrum $F(n)$, there exists a self map $\Sigma^{d} F(n) \rightarrow F(n)$, which induces a power of $v_{n}$ on $K(n)$-homology. The cofiber of this self map is then a type $(n+1)$ spectrum. This procedure allows us to construct a sequence of $F(n)$-s as iterated Moore spectra, which we may suggestively write as follows:

$$
F(n):=\mathbb{S} /\left(p^{r_{0}}, v_{1}^{r_{1}}, \ldots, v_{n-1}^{r_{n-1}}\right) .
$$

Just as localization with respect to $\mathbb{S} / p^{r}$ (for any $r$ ) has the effect of $p$-completion, one can think of the localization with respect to $F(n)$, as completion with respect to $v_{0}, \ldots, v_{n-1}$. Furthermore, localization with respect to the spectrum $T(n)=F(n)\left[v_{n}^{-1}\right]$, can be thought of as completion with respect to $v_{0}, \ldots, v_{n-1}$, followed by the inversion of $v_{n}$. It is known that the $K(n)$-localization factors through the $T(n)$-localization, and that they coincide for $M U$-modules (and also in general when $n=0,1)$. Furthermore, the localizations $\mathrm{Sp}_{T(n)}$ turn out to be independent of all the choices and thus naturally constitute another, potentially larger, candidate for the "monochromatic layers" of the chromatic height filtration. While the question of whether the inclusion $\operatorname{Sp}_{K(n)} \subseteq \operatorname{Sp}_{T(n)}$ is strict for $n \geq 2$ is open (known as the Telescope Conjecture), both candidates for the "monochromatic layers" play a pivotal role in homotopy theory.

The localizations $\operatorname{Sp}_{K(n)}$ and $\mathrm{Sp}_{T(n)}$ are known to possess several rather special and remarkable properties. Among them, the vanishing of the Tate construction for finite group actions ([Kuh04, GS96, HS96, CM17]). In [HL13], Hopkins and Lurie reinterpret this Tate vanishing property as 1semiadditivity, and vastly generalize it by showing that the $\infty$-categories $\mathrm{Sp}_{K(n)}$ are $\infty$-semiadditive. In turn, this is exploited to obtain new structural results for $\mathrm{Sp}_{K(n)}$. In [CSY18, Theorem B], the authors extended on [HL13] by classifying all the higher semiadditive localizations of Sp with respect to homotopy rings. First, for all such localizations, 1-semiadditivity was shown to be equivalent to $\infty$-semiadditivity. Second, the telescopic localizations $\mathrm{Sp}_{T(n)}$, for various primes $p$ and heights $n$, were shown to be precisely the maximal examples of such localizations (while the $\operatorname{Sp}_{K(n)}$ are the minimal). Concisely put, in the stable world, the higher semiadditive property singles out precisely the monochromatic localizations, which are parameterized by the chromatic height.

In this paper, we introduce a natural notion of semiadditive height for higher semiadditive $\infty$ categories, which in the examples $\mathrm{Sp}_{T(n)}$ and $\mathrm{Sp}_{K(n)}$ reproduces the usual chromatic height $n$, without appealing to the theory of formal groups. We then proceed to show that the semiadditive height is a fundamental invariant of a higher semiadditive $\infty$-category, which controls many aspects 
of its higher semiadditive structure, and the behavior of local systems on $\pi$-finite spaces valued in it. We also show that the semiadditive height exhibits a compelling form of the "redshift principle", where categorification has the effect of increasing the height exactly by one. When restricting to stable $\infty$-categories, we show that higher semiadditive $\infty$-categories decompose completely according to the semiadditive height, which accounts for the monochromatic nature of the higher semiadditive localizations of Sp. Finally, building on the work of Harpaz [Har17], we introduce and study universal constructions of stable $\infty$-semiadditive $\infty$-categories of height $n$, and initiate their comparison with the chromatic examples.

The present work should be viewed as part of a more extensive program that aims to place chromatic phenomena in the categorical context of the interaction between higher semiadditivity and stability. Apart from providing new tools for the study of $\mathrm{Sp}_{T(n)}$, we believe that this approach can elucidate the chromatic picture and unfold the rich and intricate structure hidden within.

\subsection{Main Results}

\section{Height Theory}

Recall that ambidexterity is a property of a space $A$ with respect to an $\infty$-category $\mathcal{C}$, that allows "integrating" $A$-families of morphisms between pairs of objects in $\mathcal{C}$ in a canonical way [HL13, Construction 4.0.7]. In particular, integrating the constant $A$-family on the identity morphism of each object, produces a natural endomorphism $|A|$ of the identity functor of $\mathcal{C}$. We call $|A|$ the $\mathcal{C}$-cardinality of $A$, and think of it as multiplication by the "size of $A$ " (the actual meaning of which depends on $\mathcal{C}$ ).

An $\infty$-category $\mathcal{C}$ is called $m$-semiadditive if every $m$-finite space is $\mathcal{C}$-ambidextrous. Our notion of semiadditive height is defined in terms of cardinalities of such spaces. For starters, let us begin with a 0 -semiadditive (i.e. semiadditive) $p$-local $\infty$-category $\mathcal{C}$. If $p$ is invertible in $\mathcal{C}$, then $\mathcal{C}$ is rational and we consider it to be of "height 0 ". In contrast, if all objects of $\mathcal{C}$ are $p$-complete, we consider it to be of "height $>0$ ". To proceed, let us assume that $\mathcal{C}$ is $m$-semiadditive for some $m \geq 0$. In such a case, we can consider the $\mathcal{C}$-cardinalities of Eilenberg-MacLane spaces:

$$
p=\left|C_{p}\right|,\left|B C_{p}\right|,\left|B^{2} C_{p}\right|, \ldots,\left|B^{m} C_{p}\right| .
$$

The definition of semiadditive height uses the maps $\left|B^{n} C_{p}\right|$ in a manner which is analogous to how the $v_{n}$-self maps are used in the definition of the chromatic height:

Definition (Semiadditive Height, 3.1.6, 3.1.11). For every $0 \leq n \leq m$, we write

(1) $\operatorname{Ht}(\mathcal{C}) \leq n$, if $\left|B^{n} C_{p}\right|$ is invertible in $\mathcal{C}$.

(2) $\operatorname{Ht}(\mathcal{C})>n$, if $\mathcal{C}$ is complete with respect to $\left|C_{p}\right|,\left|B C_{p}\right|, \ldots,\left|B^{n} C_{p}\right|^{1}$.

(3) $\operatorname{Ht}(\mathcal{C})=n$, if $\mathcal{C}$ is of height $\leq n$ and $>n-1$.

To show that the semiadditive height of $\operatorname{Sp}_{T(n)}$ and $\mathrm{Sp}_{K(n)}$ is indeed $n$, we need to get a handle on the $T(n)$-local and $K(n)$-local cardinalities of the Eilenberg-Maclane spaces $B^{k} C_{p}$. In [CSY18, Lemma 5.3.3], we have already shown that

$$
\left|B^{k} C_{p}\right|=p^{\left(\begin{array}{c}
n-1 \\
k
\end{array}\right)} \in \pi_{0} E_{n},
$$

\footnotetext{
${ }^{1}$ by Proposition 3.1.9, $\operatorname{Ht}(\mathcal{C})>n$, if and only if $\mathcal{C}$ is $\left|B^{n} C_{p}\right|$-complete.
} 
for the $\infty$-category of $K(n)$-local $E_{n}$-modules. Thus, this $\infty$-category is of height $n$. Since tensoring with $E_{n}$ is conservative on $K(n)$-local spectra, this also readily implies that $\operatorname{Sp}_{K(n)}$ is of height $n$. However, to show that $\operatorname{Sp}_{T(n)}$ is of height $n$, one has to know that the map $\pi_{0} \mathbb{S}_{T(n)} \rightarrow \pi_{0} \mathbb{S}_{K(n)}$, induced by $K(n)$-localization, detects invertibility of elements. This result was established in [CSY18, Propostion 5.1.17] using the notion of "nil-conservativity". Thus, we get that $\operatorname{Sp}_{T(n)}$ is of height $n$ as well.

The notion of semiadditive height allows us to contextualize various aspects of the $\infty$-categories $\mathrm{Sp}_{K(n)}$ and $\mathrm{Sp}_{T(n)}$ pertaining to the chromatic height. At the bottom of the hierarchy, the $\infty$ category $\mathrm{Sp}_{T(0)}=\mathrm{Sp}_{K(0)}=\mathrm{Sp}_{\mathbb{Q}}$ can be shown to be $\infty$-semiadditive by elementary arguments. This is strongly related to the fact that all connected $\pi$-finite spaces are $\mathbb{Q}$-acyclic and the cardinality of any (non-empty) $\pi$-finite space $A$ is invertible. Thus, the higher semiadditive structure of $\mathrm{Sp}_{\mathbb{Q}}$ is in a sense "trivial". The higher semiadditivity of $\mathrm{Sp}_{K(n)}$ and $\mathrm{Sp}_{T(n)}$ for $n \geq 1$ is more subtle precisely because not all connected $\pi$-finite spaces are acyclic, and not all cardinalities are invertible. One might roughly say, that the complexity of the higher semiadditive structure grows with the height. Our first main result formalizes this as follows:

Theorem A (Bounded Height, 3.2.7). Let $\mathcal{C}$ be an $n$-semiadditive $p$-local $\infty$-category, which admits all $\pi$-finite limits and colimits. If $\mathcal{C}$ is of height $\leq n$, then

(1) $\mathcal{C}$ is $\infty$-semiadditive.

(2) For every $(n-1)$-connected nilpotent $\pi$-finite space $A$, the map $|A|$ is invertible.

(3) For every $n$-connected $\pi$-finite space $A$ and $X \in \mathcal{C}$, the fold map $A \otimes X \rightarrow X$ is invertible.

(4) For every principal fiber sequence of $\pi$-finite spaces

$$
F \rightarrow A \rightarrow B
$$

if $F$ is $(n-1)$-connected and nilpotent, then $|A|=|F| \cdot|B|$.

Informally speaking, Theorem A states that the invertibility of $\left|B^{n} C_{p}\right|$ has the effect of "trivializing" the higher semiadditive structure at levels $\geq n$. In particular, it shows that it exists, which is point (1). From point (2), we deduce that having height $\leq n$ implies having height $\leq n+1$, so the conditions are of decreasing strength as the terminology suggests. Point (3) articulates a useful categorical consequence (and, in fact, a characterization) of having height $\leq n$, which does not refer directly to the higher semiadditive structure. This can be seen as a generalization of [CSY18, Theorem E], which is essentially the special case $\mathcal{C}=\mathrm{Sp}_{T(n)}$. Finally, point (4) can be used to reduce the computation of the $\mathcal{C}$-cardinalities of nilpotent $\pi$-finite spaces to those of $n$-finite ones, under the assumption that $\mathcal{C}$ is of height $\leq n$. The case $n=0$ produces an explicit formula, which recovers Baez-Dolan's classical homotopy cardinality (Example 2.2.2). We note that the possible failure of point (4) for the principal fiber sequence $B^{n-1} C_{p} \rightarrow \mathrm{pt} \rightarrow B^{n} C_{p}$, is precisely the obstruction for $\mathcal{C}$ to have height $\leq n-1$.

In their work on algebraic $K$-theory of ring spectra, Ausoni and Rognes have discovered a phenomena which they dubbed "chromatic redshift". Roughly speaking, it is the tendency of $K(R)$, which is a spectrum constructed from the $\infty$-category of perfect $R$-modules, to be of chromatic complexity larger by one, than the ring spectrum $R$ (appropriately measured). While more precise conjectures regarding this phenomena were subsequently formulated and studied, a conceptual 
source for the chromatic redshift phenomena seems to remain unrevealed. Our next result concerns an analogue of the redshift phenomena for the semiadditive height. In this context the increase by one in height is a formal consequence of categorification. To state this formally, we first note that the definition of semiadditive height makes sense for individual objects. Namely, an object $X$ in an $\infty$-semiadditive $\infty$-category $\mathcal{C}$ is of height $\leq n$ for some $n$, if $\left|B^{n} C_{p}\right|$ acts invertibly on $X$ and of height $>n$, if it is complete with respect to $\left|C_{p}\right|,\left|B C_{p}\right|, \ldots,\left|B^{n} C_{p}\right|$. Second, we exploit the fact that the $\infty$-category Cat ${ }^{\oplus-\infty}$, of $\infty$-semiadditive $\infty$-categories and $\pi$-finite colimit preserving functors, is itself $\infty$-semiadditive. Thus, given an $\infty$-semiadditive $\infty$-category $\mathcal{C}$, we can consider the height of $\mathcal{C}$ being lower equal (resp. greater than) $n$, as an object of $\mathrm{Cat}^{\oplus-\infty}$ which we shall denote by $\operatorname{ht}(\mathcal{C}) \leq n(\operatorname{resp} . \operatorname{ht}(\mathcal{C})>n)$.

Theorem B (Semiadditive Redshift, 3.3.2). Let $\mathcal{C}$ be an $\infty$-semiadditive $\infty$-category. We have that $\operatorname{Ht}(\mathcal{C}) \leq n($ resp. $\operatorname{Ht}(\mathcal{C})>n)$, if and only if $\operatorname{ht}(\mathcal{C}) \leq n+1($ resp. $\operatorname{ht}(\mathcal{C})>n+1)$.

The higher semiadditive structure of $\mathrm{Cat}^{\oplus-\infty}$ is essentially given by taking colimits over $\pi$-finite spaces. Hence, Theorem B is closely related to point (3) of Theorem A. As a concrete example, we can consider for a $T(n)$-local ring spectrum $R$, the $\infty$-category of $T(n)$-local left $R$-modules. The space of objects of this $\infty$-category is a commutative monoid for the direct sum operation. Moreover, the higher semiadditivity of the $\infty$-category of modules endows this space with a higher commutative monoid structure in the sense of [Har17, Definition 5.10]. As a consequence of Theorem B, this higher commutative monoid is of height $\leq n+1$ in the $\infty$-category of higher commutative monoids. In a future work, we shall investigate the implications of this to the chromatic redshift in algebraic $K$-theory in the sense of Ausani-Rognes.

Our main interest in the notion of higher semiadditivity is in its application to stable $\infty$-categories. As it turns out, the two properties of higher semiadditivity and stability interact in a highly nontrivial way. First and foremost, in the presence of stability, the higher semiadditive structure turns out to decompose completely according to height:

Theorem $\mathbf{C}$ (Height Decomposition, 4.2.7). Let $\mathcal{C}$ be a stable idempotent complete $m$-semiadditive $\infty$-category for some $m \in \mathbb{N}$. There is a canonical equivalence

$$
\mathcal{C} \simeq \mathcal{C}_{0} \times \cdots \times \mathcal{C}_{m-1} \times \mathcal{C}_{>m-1}
$$

were $\mathcal{C}_{0}, \ldots, \mathcal{C}_{m-1}$ and $\mathcal{C}_{>m-1}$ are the full subcategories of objects of height $0, \ldots, m-1$ and $>m-1$ respectively. ${ }^{2}$

This result sheds light on the "monochromatic nature" of higher semiadditive phenomena in the stable world. Loosely speaking, the fact that the monochromatic layers, which have different heights, glue non-trivially (by means of the chromatic fracture square), obstructs the higher semiadditivity of non-monochromatic localizations of spectra.

In view of Theorem $\mathrm{C}$, it makes sense to focus our attention on stable $\infty$-categories $\mathcal{C}$ of height exactly $n$. In [HL13, Section 5.4] it is shown that the behavior of local systems of $K(n)$-local spectra on a $\pi$-finite space $A$, strongly depends on the level of connectedness of $A$ compared with $n$. We show that some of these results hold for general stable $\infty$-categories $\mathcal{C}$ of height exactly $n$. First of all, from Theorem $\mathrm{A}(3)$, it can be deduced that for an $n$-connected $\pi$-finite space $A$, the inclusion functor $\mathcal{C} \hookrightarrow \mathcal{C}^{A}$ of constant local-systems is fully faithful. The right orthogonal

\footnotetext{
${ }^{2}$ We also treat the case $m=\infty$, which is somewhat more subtle.
} 
complement $\mathcal{C}^{\perp} \subseteq \mathcal{C}^{A}$ consists of local-systems whose global sections object (i.e. limit over $A$ ) vanishes. We prove the following:

Theorem D (Semisimplicity, 4.3.2). Let $\mathcal{C}$ be a stable $\infty$-semiadditive $\infty$-category such that $\operatorname{Ht}(\mathcal{C})=n$, and let $A$ be an $n$-connected $\pi$-finite space. There is a canonical equivalence $\mathcal{C}^{A} \simeq \mathcal{C} \times \mathcal{C}^{\perp}$.

This result can be seen as a generalization of the "semisimplicity" of $\operatorname{Sp}_{K(n)}$-valued local systems on $n$-connected $\pi$-finite spaces (compere [Lurb]). We also provide an explicit formula for the composition $\mathcal{C}^{A} \rightarrow \mathcal{C} \hookrightarrow \mathcal{C}^{A}$, as a "symmetrization" of the action of the $\infty$-group $G=\Omega A$. Intuitively, the "order" of $G$, by which one has to divide, is precisely the $\mathcal{C}$-cardinality of $G$, which is invertible by the assumption on the height of $\mathcal{C}$ and the connectivity of $A$ (Theorem A).

Based on the classification of higher semiadditive localizations of Sp with respect to homotopy rings in [CSY18, Theorem B], the authors proposed the conjecture that every stable $p$-local presentable 1 -semiadditive $\infty$-category is in fact $\infty$-semiadditive [CSY18, Conjecture 1.1.5]. In this paper, we prove a partial result in the direction of this conjecture. Given a stable $p$-local presentable $\infty$ category $\mathcal{C}$, we say that an object $X \in \mathcal{C}$ is of finite stable height if there exists a non-zero finite $p$-local spectrum $F$, such that $F \otimes X=0$. We also denote by $\mathcal{C}_{\infty^{\text {st }}} \subseteq \mathcal{C}$ the full subcategory of objects $Y \in \mathcal{C}$, for which $\operatorname{Map}(X, Y)=$ pt for all $X$ of finite stable height.

Theorem E (Bounded Bootstrap, 5.5.17). Let $\mathcal{C}$ be a stable $p$-local presentable $\infty$-category. If $\mathcal{C}$ is 1-semiadditive and $\mathcal{C}_{\infty^{\text {st }}}=0$, then it is $\infty$-semiadditive. Moreover, in this case $\mathcal{C} \simeq \prod_{n \in \mathbb{N}} \mathcal{C}_{n}$.

The condition $\mathcal{C}_{\infty^{\text {st }}}=0$ is satisfied if for example for every $X, Y \in \mathcal{C}$, the mapping spectrum $\operatorname{hom}(X, Y)$ is $L_{n}^{f}$-local for some integer $n$. The proof of Theorem E, relies on the theory of modes, which we shall review next.

\section{Mode Theory}

In [Lura, Proposition 4.8.1.15], Lurie introduced a symmetric monoidal structure on the $\infty$-category $\operatorname{Pr}^{L}$ of presentable $\infty$-categories and colimit preserving functors. Moreover, he showed that many familiar properties of presentable $\infty$-categories can be characterized as having a (necessarily unique) module structure over certain idempotent algebras in $\operatorname{Pr}^{L}$ [Lura, Section 4.8.2]. We call such idempotent presentable $\infty$-categories modes. This notion was also considered in [GGN16] from the perspective of smashing localizations of $\operatorname{Pr}^{L}$. Given a mode $\mathcal{M}$, it is a property of a presentable $\infty$-category $\mathcal{C}$ to have a structure of a module over $\mathcal{M}$. The terminology is inspired by the idea that modes classify the possible "modes of existence" in which mathematical objects can occur, manifest, and behave. Most notably, the property of stability is equivalent to having a module structure over Sp. Consequently, every stable presentable $\infty$-category is canonically enriched in Sp and colimit preserving functors between stable presentable $\infty$-categories preserve this enrichment. This structure naturally plays a significant role in the study of stable $\infty$-categories. In [Har17, Lemma 5.20], Harpaz showed that $m$-semiadditivity is similarly characterized by having a module structure over the idempotent algebra $\mathrm{CMon}_{m}$ of $m$-commutative monoids. The case $m=0$ recovers the usual $\infty$-category of commutative (i.e. $\mathbb{E}_{\infty}$ ) monoids in spaces, which classifies ordinary semiadditivity. The mapping spaces of an $m$-semiadditive $\infty$-category obtain a canonical $m$-commutative monoid structure, by analogy with the Sp-enrichment of stable $\infty$-categories.

In the final section of this paper, we develop the theory of modes further and apply it to the study of height in stable presentable higher semiadditive $\infty$-categories. First, by the general theory of 
modes, $\mathrm{CMon}_{m} \otimes \mathrm{Sp}$ is also a mode, which classifies the property of being at the same time stable and $m$-semiadditive. Furthermore, using Theorem $\mathrm{C}$, we show:

Theorem $\mathbf{F}$ (5.3.6). For every $n \geq 0$, there exists a mode $\mathbf{3}_{n}{ }^{3}$, which classifies the property of being stable, $p$-local, $\infty$-semiadditive and of height $n$.

It is natural to compare $\boldsymbol{צ}_{n}$ with $\mathrm{Sp}_{T(n)}$, which is in a sense the universal $p$-local height $n$ localization of spectra. Since $\operatorname{Sp}_{T(n)}$ is also $\infty$-semiadditive and of semiadditive height $n$, the theory of modes implies the existence of a unique colimit preserving symmetric monoidal functor $L: \mathbf{צ}_{n} \rightarrow \mathrm{Sp}_{T(n)}$. In the case $n=0$, the functor $L: \boldsymbol{צ}_{0} \rightarrow \mathrm{Sp}_{T(0)}$ is an equivalence and hence $\boldsymbol{צ}_{0} \simeq \mathrm{Sp}_{\mathbb{Q}}$ (Example 5.3.7). In general, we show that $L$ exhibits $\mathrm{Sp}_{T(n)}$ as a smashing localization of $\boldsymbol{\Xi}_{n}$ in the sense that $L$ admits a fully faithful right adjoint $\operatorname{Sp}_{T(n)} \hookrightarrow \mathbf{צ}_{n}$ and there is a canonical isomorphism $L X \simeq \mathbb{S}_{T(n)} \otimes X$ for all $X \in \boldsymbol{צ}_{n}$ (Corollary 5.5.14). For $n \geq 1$, the $\infty$-category $\boldsymbol{צ}_{n}$ also resembles $\operatorname{Sp}_{T(n)}$ in that the unique colimit preserving symmetric monoidal functor $\mathrm{Sp} \rightarrow \boldsymbol{צ}_{n}$ vanishes on all bounded above spectra (Proposition 5.3.9), and that the right adjoint of the unique colimit preserving symmetric monoidal functor $\mathcal{S} \rightarrow \boldsymbol{צ}_{n}$ is conservative (Corollary 5.3.10). We consider $\boldsymbol{צ}_{n}$ to be a natural extension of $\mathrm{Sp}_{T(n)}$, which is a universal home for phenomena of height $n$.

In a previous draft of this paper, we proposed the conjecture that for every $n \geq 0$, the unique colimit preserving symmetric monoidal functor $L: \boldsymbol{\Xi}_{n} \rightarrow \mathrm{Sp}_{T(n)}$ is an equivalence. However, this conjecture was soon disproved by Allen Yuan already in the case $n=1$. More precisely, using the Segal Conjecture (now a theorem [Car84]), he has constructed a higher commutative monoid structure of height 1 on the $p$-complete sphere, as an object of the $\infty$-category of $p$-complete spectra. The details and some interesting applications of this example will appear in a separate paper by him.

Finally, the theory of modes allows us not only to analyze the implications of certain properties of presentable $\infty$-categories, but also to enforce them in a universal way. For every mode $\mathcal{M}$ and a presentable $\infty$-category $\mathcal{C}$, we can view $\mathcal{M} \otimes \mathcal{C}$ as the universal approximation of $\mathcal{C}$ by a presentable $\infty$-category which satisfies the property classified by $\mathcal{M}$. For example, $\operatorname{Sp} \otimes \mathcal{C} \simeq \operatorname{Sp}(\mathcal{C})$ is the stabilization of $\mathcal{C}$ [Lura, Example 4.8.1.23], and similarly, $\mathrm{CMon}_{m} \otimes \mathcal{C}$ is the " $m$-semiadditivization" of $\mathcal{C}$ [Har17, Corollary 5.18]. As alluded to above, the non-trivial gluing in the chromatic fracture square, prevents $L_{n}^{f}$ Sp from being higher semiadditive for $n \geq 1$. Employing the additive $p$ derivation $\delta$ on the rings $\pi_{0} \mathbb{S}_{T(n)}$ constructed in [CSY18, Section 4], we show that forcing even 1-semiadditivity on $L_{n}^{f} \mathrm{Sp}$, has the effect of "dissolving the glue" in the chromatic fracture squares:

Theorem G (1-Semiadditive Splitting, 5.4.10). For every $n \geq 0$, there is a unique equivalence of presentably symmetric monoidal $\infty$-categories

$$
\mathrm{CMon}_{1} \otimes L_{n}^{f} \mathrm{Sp} \simeq \prod_{k=0}^{n} \mathrm{Sp}_{T(k)} .
$$

In particular, we see that forcing 1-semiadditivity on $L_{n}^{f}$ Sp makes it automatically $\infty$-semiadditive. Noticing that both sides of Theorem G are modes, we can reinterpret it in terms of the properties classified by them. Namely, that every 1 -semiadditive stable presentable $\infty$-category whose mapping spectra are $L_{n}^{f}$-local, is $\infty$-semiadditive. With some additional effort, we deduce from it the stronger statement of Theorem E.

\footnotetext{
${ }^{3}$ The letter $\mathbf{Y}$ (pronounced "tsadi") is the first letter in the Hebrew word for "color". The notation was chosen to indicate the close relationship with chromatic homotopy theory.
} 


\subsection{Organization}

We shall now outline the content of each section of the paper.

In section 2, we recall and expand the theory of higher semiadditivity. We discuss the notion of cardinality for a $\pi$-finite space in a higher semiadditive $\infty$-category and the corresponding notion of amenability. We then give several examples of these notions in various higher semiadditive $\infty$ categories, and relate the notion of amenability to the behavior of local systems, through the notion of acyclicity.

In section 3, we discuss the main notion of this paper, that of height in a higher semiadditive $\infty$ category, defined in terms of the cardinalities of Eilenberg-MacLane spaces. We show that the higher semiadditive structure trivializes above the height (Theorem A) and exhibits a redshift principle of increasing by one under categorification (Theorem B).

In section 4 , we study semiadditivity and height for stable $\infty$-categories. After a general discussion on recollement, we show that a stable higher semiadditive $\infty$-category splits as a product according to height (Theorem $\mathrm{C}$ ). We then study local systems valued in a stable higher semiadditive $\infty$-category of height $n$ and show how the notion of height is related to the phenomenon of semisimplicity of local systems (Theorem D). Finally, we use nil-conservative functors to show that semiadditive and chromatic height coincide for monochromatic localizations of spectra.

In section 5, we study the theory of modes, i.e. that of idempotent algebras in the category of presentable $\infty$-categories. We show how algebraic operations on modes, such as tensor product and localization, translate into operations on the properties of presentable $\infty$-categories classified by them. We then show that the main notions studied in this paper, higher semiadditivity and height, together with the more classical notion of chromatic height, are all encoded by modes (e.g. Theorem F). Using this theory, we study the interaction between the chromatic and the semiadditive heights through the interactions between the corresponding modes. In particular, we prove Theorem $\mathrm{G}$ and deduce from it Theorem E.

\subsection{Conventions}

Throughout the paper, we work in the framework of $\infty$-categories (a.k.a. quasicategories), and in general follow the notation of [Lur09] and [Lura]. We shall also use the following terminology and notation most of which is consistent with [CSY18]:

(1) We slightly diverge from [Lur09] and [Lura] in the following points:

(a) We use the term isomorphism for an invertible morphism in an $\infty$-category (i.e. equivalence).

(b) We denote by $\mathcal{C} \simeq \subseteq \mathcal{C}$ the maximal $\infty$-subgroupoid of an $\infty$-category $\mathcal{C}$.

(c) We write Pr for the $\infty$-category of presentable $\infty$-categories and colimit preserving functors denoted in [Lur09] by $\operatorname{Pr}^{L}$.

(d) We denote by Cat st $_{\text {s }} \subset$ Cat the subcategory spanned by stable $\infty$-categories and exact functors. Similarly, we denote by $\operatorname{Pr}_{\text {st }} \subseteq \operatorname{Pr}$ the full subcategory spanned by stable presentable $\infty$-categories.

(2) We say that a space $A \in \mathcal{S}$ is 
(a) $m$-finite for $m \geq-2$, if $m=-2$ and $A$ is contractible, or $m \geq-1$, the set $\pi_{0} A$ is finite and all the fibers of the diagonal map $\Delta: A \rightarrow A \times A$ are $(m-1)$-finite ${ }^{4}$.

(b) $\pi$-finite or $\infty$-finite, if it is $m$-finite for some integer $m \geq-2$. For $-2 \leq m \leq \infty$, we denote by $\mathcal{S}_{m \text {-fin }} \subseteq \mathcal{S}$ the full subcategory spanned by $m$-finite spaces.

(c) $p$-space, if all the homotopy groups of $A$ are $p$-groups.

(3) Given an $\infty$-category $\mathcal{C} \in$ Cat $_{\infty}$,

(a) For every map of spaces $A \stackrel{q}{\rightarrow} B$, we write $q^{*}: \mathcal{C}^{B} \rightarrow \mathcal{C}^{A}$ for the pullback functor and $q$ ! and $q_{*}$ for the left and right adjoints of $q^{*}$ whenever they exist.

(b) Whenever convenient we suppress the canonical equivalence of $\infty$-categories $\mathcal{S} / \mathrm{pt} \stackrel{\sim}{\longrightarrow} \mathcal{S}$ by identifying a space $A$ with the terminal map $A \stackrel{q}{\rightarrow}$ pt. In particular, for every $\infty$ category $\mathcal{C}$, we write $A^{*}$ for $q^{*}$ and similarly $A_{\text {! }}$ and $A_{*}$ for $q$ ! and $q_{*}$ whenever they exist.

(c) For every $X \in \mathcal{C}$ we write $X[A]$ for $A_{!} A^{*} X$ and denote the fold (i.e. counit) map by $X[A] \stackrel{\nabla}{\rightarrow} X$. Similarly, we write $X^{A}$ for $A_{*} A^{*} X$ and denote the diagonal (i.e. unit) map by $X \stackrel{\Delta}{\rightarrow} X^{A}$.

(4) Given a map of spaces $q: A \rightarrow B$, we denote for every $b \in B$, the homotopy fiber of $q$ over $b$ by $q^{-1}(b)$. We say that

(a) an $\infty$-category $\mathcal{C}$ admits all $q$-limits (resp. $q$-colimits) if it admits all limits (resp. colimits) of shape $q^{-1}(b)$ for all $b \in B$.

(b) a functor $F: \mathcal{C} \rightarrow \mathcal{D}$ preserves $q$-colimits (resp. $q$-limits) if it preserves all colimits (resp. limits) of shape $q^{-1}(b)$ for all $b \in B$.

(5) For every $-2 \leq m \leq \infty$,

(a) by $m$-finite (co)limits we mean (co)limits indexed by an $m$-finite space.

(b) We let $\mathrm{Cat}_{m \text {-fin }} \subset \mathrm{Cat}_{\infty}$ (resp. Cat ${ }^{m \text {-fin }} \subset \mathrm{Cat}_{\infty}$ ) be the subcategory spanned by $\infty$ categories which admit $m$-finite colimits (resp. limits) and functors preserving them.

(c) For $\mathcal{C}, \mathcal{D} \in \mathrm{Cat}_{m \text {-fin }}\left(\right.$ resp. $\left.\mathrm{Cat}^{m \text {-fin }}\right)$ we write $\operatorname{Fun}_{m \text {-fin }}(\mathcal{C}, \mathcal{D})\left(\operatorname{resp} . \operatorname{Fun}^{m \text {-fin }}(\mathcal{C}, \mathcal{D})\right)$ for the full subcategory of $\operatorname{Fun}(\mathcal{C}, \mathcal{D})$ spanned by the $m$-finite colimit (resp. limit) preserving functors.

(d) We let $\mathrm{Cat}^{\oplus-m} \subset \mathrm{Cat}_{\infty}$ be the subcategory spanned by the $m$-semiadditive $\infty$-categories and $m$-semiadditive (i.e. $m$-finite colimit preserving) functors.

(e) Given an $\infty$-operad $\mathcal{O}$, we say that $\mathcal{C} \in \operatorname{Alg}_{\mathcal{O}}\left(\mathrm{Cat}_{\infty}\right)$ is compatible with $\mathcal{K}$-indexed colimits for some collection of $\infty$-categories $\mathcal{K}$, if $\mathcal{C}$ admits $\mathcal{K}$-indexed colimits and every tensor operation $\otimes: \mathcal{C}^{n} \rightarrow \mathcal{C}$ of $\mathcal{O}$ preserves $\mathcal{K}$-indexed colimits in each variable.

(f) An $m$-semiadditively $\mathcal{O}$-monoidal $\infty$-category is an $\mathcal{O}$-monoidal $m$-semiadditive $\infty$-category which is compatible with $m$-finite colimits.

\footnotetext{
${ }^{4}$ For $m \geq 0$, this is equivalent to $A$ having finitely many components, each of them $m$-truncated with finite homotopy groups.
} 
(6) If $\mathcal{C}$ is a monoidal $\infty$-category and $\mathcal{D}$ is an $\infty$-category enriched in $\mathcal{C}$, we write $\operatorname{hom}_{\mathcal{D}}^{\mathcal{C}}(X, Y)$ for the $\mathcal{C}$-mapping object of $X, Y \in \mathcal{D}$. We omit the subscript or superscript when they are understood from the context. In particular, when $\mathcal{C}$ is closed, $\operatorname{hom}_{\mathcal{C}}(X, Y)$ means $\operatorname{hom}_{\mathcal{C}}^{\mathcal{C}}(X, Y)$. For every $\infty$-category $\mathcal{C}$ we have $\operatorname{hom}_{\mathcal{C}}^{\mathcal{S}}(X, Y)=\operatorname{Map}_{\mathcal{C}}(X, Y)$.

\subsection{Acknowledgments}

We would like to thank Tobias Barthel, Clark Barwick, Agnès Beaudry, Jeremy Hahn, Gijs Heuts, Mike Hopkins, and Tyler Lawson for useful discussions. We thank Allen Yuan for sharing with us his ideas regarding the topics of this paper. We also like to thank the entire Seminarak group, especially Shay Ben Moshe, for useful comments on the paper's first draft.

The first author is supported by the Adams Fellowship Program of the Israel Academy of Sciences and Humanities. The second author is supported by ISF1588/18 and BSF 2018389.

\section{Semiadditivity}

In this section, we collect general facts regarding the notion of ambidexterity and its implications. We begin by reviewing some background material and most importantly (re)introduce the notion of cardinality for ambidextrous $\pi$-finite spaces. We provide a variety of examples of cardinality in both the stable and the unstable settings, including those of relevance to chromatic homotopy theory. Of particular importance are the amenable spaces, whose cardinality is invertible. We continue the study of such spaces, which we began in [CSY18, Section 3], and in particular, establish its implications for the behavior of certain $\pi$-finite limits and colimits. In the next section, the amenability of Eilenberg-MacLane spaces will play a central role in the definition of "semiadditive height" for higher semiadditive $\infty$-categories, which is the main subject of this paper.

\subsection{Preliminaries}

In this subsection, we review some basic definitions and facts regarding ambidexterity from [HL13, Section 4], cardinality from [CSY18], and higher commutative monoids from [Har17, Section 5.2]. This subsection serves mainly to set up notation, terminology, and a convenient formulation of fundamental results.

\section{Ambidexterity}

Recall from [HL13, Section 4.1] the definition of ambidexterity:

Definition 2.1.1. Let $\mathcal{C} \in \mathrm{Cat}_{\infty}$. A $\pi$-finite map $A \stackrel{q}{\rightarrow} B$ is called:

(1) weakly $\mathcal{C}$-ambidextrous if it is an isomorphism, or $A \stackrel{\Delta_{q}}{\longrightarrow} A \times_{B} A$ is $\mathcal{C}$-ambidextrous.

(2) $\mathcal{C}$-ambidextrous if it is weakly $\mathcal{C}$-ambidextrous, $\mathcal{C}$ admits all $q$-limits and $q$-colimits and the the norm map $q_{!} \stackrel{\mathrm{Nm}_{q}}{\longrightarrow} q_{*}$ is an isomorphism. 
Definition 2.1.1 should be understood inductively on the level of truncatedness of $q$. A (-2)-finite map, i.e. an isomorphism, is always $\mathcal{C}$-ambidextrous. If $q$ is $m$-finite, then the diagonal map

$$
A \stackrel{\Delta_{q}}{\longrightarrow} A \times_{B} A
$$

is $(m-1)$-finite and the ambidexterity of $\Delta_{q}$ allows in turn the definition of $\mathrm{Nm}_{q}$ by [HL13, Construction 4.1.8] (see also [CSY18, Definition 3.1.3]).

Remark 2.1.2. A map $A \stackrel{q}{\rightarrow} B$ is $\mathcal{C}$-ambidextrous if and only if all the fibers of $q$ are $\mathcal{C}$-ambidextrous spaces [HL13, Corollary 4.2.6(2), Corollary 4.3.6]. Moreover, the fibers of the diagonal $A \rightarrow A \times A$ are the path spaces of $A$. In other words, $A$ is weakly $\mathcal{C}$-ambidextrous if and only if the path spaces of $A$ are $\mathcal{C}$-ambidextrous. Thus, $\mathcal{C}$-ambidexterity is ultimately a property of spaces.

By [HL13], the property of ambidexterity has the following useful characterization, which avoids the explicit inductive construction of the norm map:

Proposition 2.1.3. Let $\mathcal{C}$ be an $\infty$-category and let $A \stackrel{q}{\rightarrow} B$ be a $\pi$-finite map. The map $q$ is $\mathcal{C}$-ambidextrous if and only if the following hold:

(1) $q$ is weakly $\mathcal{C}$-ambidextrous.

(2) $\mathcal{C}$ admits all q-limits and q-colimits.

(3) Either $q_{*}$ preserves all q-colimits or $q$ ! preserves all q-limits.

Proof. By Remark 2.1.2, we may assume that $B=\mathrm{pt}$, in which case it is essentially [HL13, Proposition 4.3.9] and its dual [HL13, Remark 4.3.10]. We note that while the claim in [HL13] is stated under the stronger assumption that $\mathcal{C}$ admits, and $q_{*}$ (resp. $q_{\text {! }}$ ) preserves, all small colimits (resp. limits), the proof uses only $q$-colimits (resp. $q$-limits).

As a consequence, we can easily deduce that ambidexterity enjoys the following closure properties with respect to the $\infty$-category:

Proposition 2.1.4. Let $\mathcal{C}$ be an $\infty$-category and let $A$ be a $\pi$-finite $\mathcal{C}$-ambidextrous space. The space $A$ is also $\mathcal{D}$-ambidextrous for:

(1) $\mathcal{D}=\mathcal{C}^{\text {op }}$ the opposite $\infty$-category of $\mathcal{C}$.

(2) $\mathcal{D}=\operatorname{Fun}(\mathcal{I}, \mathcal{C})$ for an $\infty$-category $\mathcal{I}$.

(3) $\mathcal{D} \subseteq \mathcal{C}$ containing the final object and closed under $\Omega_{a}^{k} A$-limits for all $a \in A$ and $k \geq 0$.

(4) $\mathcal{D} \subseteq \mathcal{C}$ containing the initial object and closed under $\Omega_{a}^{k} A$-colimits for all $a \in A$ and $k \geq 0$.

Proof. First, (4) follows from (3) and (1), so it suffices to consider (1)-(3). In all cases, we proceed by induction on $m$, so we may assume by induction that $A$ is weakly $\mathcal{D}$-ambidextrous. By Proposition 2.1.3, it suffices to verify that $A$-limits and $A$-colimit exist in $\mathcal{D}$ and that the functor $A_{*}$ preserves $A$-colimits or that $A_{\text {! }}$ preserves $A$-limits. For (1), the claim follows from the fact that limits in $\mathcal{C}^{o p}$ are computed as colimits in $\mathcal{C}$ and vice versa. For (2), we use the fact that limits and colimits in $\operatorname{Fun}(\mathcal{I}, \mathcal{C})$ are computed pointwise. For $(3)$, since $A$-limits in $\mathcal{C}$ coincide with $A$-colimits in $\mathcal{C}$, it follows that $A$-limits and $A$-colimits are computed in $\mathcal{D}$ in the same way as in $\mathcal{C}$. 


\section{Cardinality}

The main feature of ambidexterity is that it allows us to integrate families of morphisms in $\mathcal{C}$. That is, given a $\mathcal{C}$-ambidextrous map $A \stackrel{q}{\rightarrow} B$ and $X, Y \in \mathcal{C}^{B}$ we have a map (see [CSY18, Definition 2.1.11])

$$
\int_{q}: \operatorname{Map}_{\mathcal{C}}\left(q^{*} X, q^{*} Y\right) \rightarrow \operatorname{Map}_{\mathcal{C}}(X, Y)
$$

When $B=$ pt, we can think of an element of $\operatorname{Map}_{\mathcal{C}}\left(q^{*} X, q^{*} Y\right)$ as a map $A \stackrel{f}{\rightarrow} \operatorname{Map}_{\mathcal{C}}(X, Y)$, and of $\int_{A} f \in \operatorname{Map}_{\mathcal{C}}(X, Y)$ as the sum of $f$ over the points of $A$. In particular, we can integrate the identity morphism:

Definition 2.1.5. Let $\mathcal{C} \in \mathrm{Cat}_{\infty}$ and let $A \stackrel{q}{\rightarrow} B$ be a $\mathcal{C}$-ambidextrous map. We have a natural transformation $\operatorname{Id}_{\mathcal{C}^{B}} \stackrel{|q|_{\mathcal{C}}}{\longrightarrow} \operatorname{Id}_{\mathcal{C}^{B}}$ given by the composition

$$
\operatorname{Id}_{\mathcal{C}^{B}} \stackrel{u_{*}}{\longrightarrow} q_{*} q^{*} \stackrel{\mathrm{Nm}_{q}^{-1}}{\longrightarrow} q ! q^{*} \stackrel{c_{!}}{\longrightarrow} \operatorname{Id}_{\mathcal{C}^{B}} .
$$

For a $\mathcal{C}$-ambidextrous space $A$, we write $\operatorname{Id}_{\mathcal{C}} \stackrel{|A|_{\mathcal{C}}}{\longrightarrow} \operatorname{Id}_{\mathcal{C}}$ and call $|A|_{\mathcal{C}}$ the $\mathcal{C}$-cardinality of $A$.

The name "C $\mathcal{C}$-cardinality" can be explained as follows. For a given object $X \in \mathcal{C}$, the map $X \stackrel{|A|_{X}}{\longrightarrow} X$ equals $\int_{A} \operatorname{Id}_{X}$. Thus, we think of $|A|_{X}$ as the sum of the identity of $X$ with itself " $A$ times". Or in other words, as the result of multiplying by the "cardinality of $A$ " on $X$. The basic example which motivates the terminology and notation is the following:

Example 2.1.6. Let $\mathcal{C}$ be a semiadditive $\infty$-category. For a finite set $A$, viewed as a 0 -finite space, the operation $|A|_{\mathcal{C}}$ is simply the multiplication by the natural number, which is the cardinality of $A$ in the usual sense.

Remark 2.1.7. For a general $\mathcal{C}$-ambidextrous map $A \stackrel{q}{\rightarrow} B$, the transformation $|q|_{\mathcal{C}}$ can be understood as follows. Let $B \stackrel{X}{\longrightarrow} \mathcal{C}$ be a $B$-family of objects in $\mathcal{C}$. For each $b \in B$, let us denote the fiber of $q$ at $b$ by $A_{b}$ and the evaluation of $X$ at $b$ by $X_{b}$. By [CSY18, Proposition 3.1.13], the map $X \stackrel{|q|_{\mathcal{C}}}{\longrightarrow} X$ acts on each $X_{b}$ by $\left|A_{b}\right|_{\mathcal{C}}$. In other words, $|q|_{\mathcal{C}}$ is just the $B$-family of $\mathcal{C}$-cardinalities of the $B$-family of spaces $A_{b}$.

For a $\mathcal{C}$-ambidextrous space $A$, the $A$-limits and $A$-colimits in $\mathcal{C}$ are canonically isomorphic. This can be used to show the following:

Proposition 2.1.8. Let $\mathcal{C}, \mathcal{D} \in \mathrm{Cat}_{\infty}$ and let $A$ be a $\mathcal{C}$ - and $\mathcal{D}$-ambidextrous space. A functor $F: \mathcal{C} \rightarrow \mathcal{D}$ preserves all $A$-limits if and only if it preserves all $A$-colimits. Moreover, if $F$ preserves all $A$-(co)limits, then $F\left(|A|_{\mathcal{C}}\right)=|A|_{\mathcal{D}}$.

Proof. [CSY18, Corollary 3.2.4] shows that $F$ preserves $m$-finite limits if and only if it preserves $m$-finite colimits and [CSY18, Corollary 3.2.7] shows that in this case $F\left(|A|_{\mathcal{C}}\right)=|A|_{\mathcal{D}}$ for every $m$-finite space $A$. One easily checks that all the arguments are valid for an individual space $A$ as well.

The $\mathcal{C}$-cardinality is additive in the following sense: 
Proposition 2.1.9. Let $\mathcal{C} \in \mathrm{Cat}_{\infty}$ and $A \stackrel{q}{\rightarrow} B$ be a map of spaces. If $B$ and $q$ are $\mathcal{C}$-ambidextrous then $A$ is $\mathcal{C}$-ambidextrous and for every $X \in \mathcal{C}$,

$$
|A|_{X}=\int_{B}|q|_{B^{*} X}
$$

Informally, Proposition 2.1.9 says that the cardinality of the total space $A$ is the "sum over $B$ " of the cardinalities of the fibers $A_{b}$ of $q$.

Proof. This follows from the Higher Fubini's Theorem ([CSY18, Propostion 2.1.15]) applied to the identity morphism.

Remark 2.1.10. By Proposition 2.1 .9 and Remark 2.1.7, for every pair of $\mathcal{C}$-ambidextrous spaces $A$ and $B$ we have

$$
|A \times B|_{\mathcal{C}}=|A|_{\mathcal{C}}|B|_{\mathcal{C}} \in \operatorname{End}\left(\operatorname{Id}_{\mathcal{C}}\right) .
$$

Similarly, by [HL13, Remark 4.4.11], for every $\mathcal{C}$-ambidextrous space $A$ we have

$$
|A|_{\mathcal{C}}=\sum_{a \in \pi_{0} A}\left|A_{a}\right|_{\mathcal{C}} \in \operatorname{End}\left(\operatorname{Id}_{\mathcal{C}}\right)
$$

The various naturality properties enjoyed by the operations $|A|_{\mathcal{C}}$ allow for useful abuses of notation:

(1) Given an $A$-colimit preserving functor $F: \mathcal{C} \rightarrow \mathcal{D}$, if $A$ is $\mathcal{C}$ - and $\mathcal{D}$-ambidextrous, we get by Proposition 2.1.8 that $F\left(|A|_{\mathcal{C}}\right)=|A|_{\mathcal{D}}$. We therefore write $|A|$, dropping the subscript $\mathcal{C}$, whenever convenient. As a special case, let $\mathcal{C}_{\circ} \subseteq \mathcal{C}$ be a full subcategory which is closed under $A$-(co)limits. The cardinality $|A|_{\mathcal{C}_{\circ}}$ coincides with the restriction of $|A|_{\mathcal{C}}$ to $\mathcal{C}_{\circ}$.

(2) When $\mathcal{C}$ is monoidal and the tensor product preserves $A$-colimits in each variable, the action of $|A|$ on any object $X \in \mathcal{C}$ can be identified with tensoring $\mathbb{1} \stackrel{|A|}{\longrightarrow} \mathbb{1}$ with $X$ (see [CSY18, Lemma 3.3.4]). We therefore sometimes identify $|A|$ with an element of $\pi_{0} \mathbb{1}:=\pi_{0} \operatorname{Map}(\mathbb{1}, \mathbb{1})$.

(3) Furthermore, for $R \in \operatorname{Alg}(\mathcal{C})$, the map $R \stackrel{|A|}{\longrightarrow} R$ can be also identified with multiplication by the image of $|A| \in \pi_{0} \mathbb{1}$ under the map $\pi_{0} \mathbb{1} \rightarrow \pi_{0} R$, which we also denote by $|A|$.

All these abuses of notation are compatible with standard conventions when $A$ is a finite set (see Example 2.1.6).

\section{Higher commutative monoids}

Of particular interest are $m$-semiadditive $\infty$-categories, i.e. those for which all $m$-finite spaces are ambidextrous. For $m=0$, we recover the ordinary notion of a semiadditive $\infty$-category. The central feature of semiadditive $\infty$-categories is the existence of a canonical summation operation on their spaces of morphisms, endowing them with a commutative monoid structure. In [Har17, Section 5.2], an analogous theory of $m$-commutative monoids is developed and applied to the study of $m$-semiadditivity for all $-2 \leq m<\infty$. In this section, we recall from [Har17] a part of this theory of higher commutative monoids and extend it to the case $m=\infty$. 
Definition 2.1.11. [Har17, Definition 5.10, Proposition 5.14] Let $-2 \leq m<\infty$, for $\mathcal{C} \in \mathrm{Cat}^{m \text {-fin }}$, the $\infty$-category of $m$-commutative monoids in $\mathcal{C}$ is given by

$$
\mathrm{CMon}_{m}(\mathcal{C}):=\operatorname{Fun}^{m-\text { fin }}\left(\operatorname{Span}\left(\mathcal{S}_{m \text {-fin }}\right)^{o p}, \mathcal{C}\right) .
$$

In the case $\mathcal{C}=\mathcal{S}$, we simply write $\mathrm{CMon}_{m}$ and refer to its objects as $m$-commutative monoids ${ }^{5}$.

In the case $m=-2$, evaluating at pt, the unique object of $\operatorname{Span}\left(\mathcal{S}_{(-2) \text {-fin }}\right)$, gives an equivalence $\mathrm{CMon}_{-2}(\mathcal{C}) \simeq \mathcal{C}$.

Remark 2.1.12. We can understand the definition of $\mathrm{CMon}_{m}$ as follows. An object $X \in \mathrm{CMon}_{m}$ consists of the "underlying space" $X(\mathrm{pt})$, together with a collection of coherent operations for summation of $m$-finite families of points in it. Indeed, for every $A \in \mathcal{S}_{m \text {-fin }}$, there is a canonical equivalence $X(A) \simeq X(\mathrm{pt})^{A}$. Given $A \rightarrow B$ in $\mathcal{S}_{m \text {-fin }}$, the "right way" map $X^{B} \rightarrow X^{A}$ is given simply by restriction, while the "wrong way" map $X^{A} \rightarrow X^{B}$ encodes integration along the fibers. The functoriality with respect to composition of spans encodes the coherent associativity and commutativity of these integration operations.

Higher commutative monoids of different levels are related by "forgetful functors".

Proposition 2.1.13. Let $-2 \leq m<\infty$ and let $\mathcal{C} \in \mathrm{Cat}^{(m+1)-\mathrm{fin}}$. The restriction along the inclusion functor

$$
\iota_{m}: \operatorname{Span}\left(\mathcal{S}_{m-\text { fin }}\right) \hookrightarrow \operatorname{Span}\left(\mathcal{S}_{(m+1) \text {-fin }}\right)
$$

induces a limit preserving functor

$$
\iota_{m}^{*}: \mathrm{CMon}_{m+1}(\mathcal{C}) \rightarrow \mathrm{CMon}_{m}(\mathcal{C}) .
$$

Proof. It suffices to show that the $\iota_{m}$ preserves $m$-finite colimits. By [Har17, Corollary 2.16], it suffices to show that the composite

$$
F: \mathcal{S}_{m \text {-fin }} \rightarrow \operatorname{Span}\left(\mathcal{S}_{m \text {-fin }}\right) \hookrightarrow \operatorname{Span}\left(\mathcal{S}_{(m+1) \text {-fin }}\right)
$$

preserves $m$-finite colimits. Indeed, $F$ factors also as the composite

$$
\mathcal{S}_{m \text {-fin }} \hookrightarrow \mathcal{S}_{(m+1) \text {-fin }} \rightarrow \operatorname{Span}\left(\mathcal{S}_{(m+1) \text {-fin }}\right) .
$$

The first functor clearly preserves $m$-finite colimits while the second one preserves $m$-finite colimits by [Har17, Proposition 2.12].

We now extend the definition of $\mathrm{CMon}_{m}$ to $m=\infty$.

Definition 2.1.14. For $\mathcal{C} \in \mathrm{Cat}^{\infty-\mathrm{fin}}$, we denote

$$
\mathrm{CMon}_{\infty}(\mathcal{C}):=\lim _{m} \operatorname{CMon}_{m}(\mathcal{C}) .{ }^{6}
$$

As above we write $\mathrm{CMon}_{\infty}:=\mathrm{CMon}_{\infty}(\mathcal{S})$.

\footnotetext{
${ }^{5}$ For $m=0$, one indeed recovers the usual notion of a commutative (i.e. $\mathbb{E}_{\infty}$ ) monoids in spaces by comparison with Segal objects [Lura, Section 2.4.2].

${ }^{6}$ In fact one can define $\mathrm{CMon}_{\infty}(\mathcal{C})$ by substituting $m=\infty$ in Definition 2.1.11 and obtain an equivalent notion (we shall not use this fact).
} 
When $\mathcal{C}$ is presentable, $\mathrm{CMon}_{m}(\mathcal{C})$ is presentable for all $m$, by [Har17, Lemma 5.17]. Moreover, $\mathrm{CMon}_{\infty}(\mathcal{C})$ can then be described as the colimit of $\mathrm{CMon}_{m}(\mathcal{C})$ in $\operatorname{Pr}$.

Lemma 2.1.15. For $\mathcal{C} \in \operatorname{Pr}$, the forgetful functors

$$
\iota_{m}^{*}: \mathrm{CMon}_{m+1}(\mathcal{C}) \rightarrow \mathrm{CMon}_{m}(\mathcal{C}),
$$

admit left adjoints and the colimit of the sequence

$$
\mathcal{C} \simeq \mathrm{CMon}_{-2} \rightarrow \mathrm{CMon}_{-1}(\mathcal{C}) \rightarrow \cdots \rightarrow \mathrm{CMon}_{m}(\mathcal{C}) \rightarrow \cdots
$$

in $\operatorname{Pr}$ is $\mathrm{CMon}_{\infty}(\mathcal{C})$. In particular, $\mathrm{CMon}_{\infty}(\mathcal{C})$ is presentable.

Proof. Since $\mathrm{CMon}_{m}(\mathcal{C})$ is presentable for all $m$, by the adjoint functor theorem [Lur09, Corollary 5.5.2.9], the functor $\iota_{m}^{*}$ admits a left adjoint if and only if it is accessible and limit preserving. The functor $\iota_{m}^{*}$ is $\kappa$-accessible for any $\kappa$ large enough such that $(m+1)$-finite limits commute with $\kappa$-filtered colimits in $\mathcal{C}$, and by Proposition 2.1.13, is limit preserving. The second claim follows from the description of colimts in Pr (see [Lur09, Theorem 5.5.3.18, Corollary 5.5.3.4]).

In the theory of $m$-semiadditivity, the $\infty$-category $\mathrm{CMon}_{m}$ plays an analogous role to that of the $\infty$-category CMon of commutative (i.e. $\mathbb{E}_{\infty}$ ) monoids in spaces, in the the theory of ordinary (i.e. 0 ) semiadditivity. In particular, the mapping space between every two objects in an $m$-semiadditive $\infty$-category has a canonical $m$-commutative monoid structure. To see this, we begin by recalling the fundamental universal property of $\mathrm{CMon}_{m}$ from [Har17]. For each $\mathcal{C} \in \mathrm{Cat}^{m \text {-fin }}$, we have a forgetful functor $\mathrm{CMon}_{m}(\mathcal{C}) \rightarrow \mathrm{CMon}_{-2}(\mathcal{C})=\mathcal{C}$, given by evaluation at pt $\in \mathcal{S}_{m \text {-fin }}$.

Proposition 2.1.16. Let $-2 \leq m \leq \infty$. For every $\mathcal{C} \in \mathrm{Cat}^{\oplus-m}$ and $\mathcal{D} \in \mathrm{Cat}^{m \text {-fin }}$, post-composition with the forgetful functor induces an equivalence of $\infty$-categories

$$
\operatorname{Fun}^{m-\operatorname{fin}}\left(\mathcal{C}, \operatorname{CMon}_{m}(\mathcal{D})\right) \simeq \operatorname{Fun}^{m-\text { fin }}(\mathcal{C}, \mathcal{D}) .
$$

Proof. The case $m<\infty$ is proved in [Har17, Proposition 5.14]. For $m=\infty$ we have

$$
\begin{aligned}
& \operatorname{Fun}^{\infty \text {-fin }}\left(\mathcal{C}, \operatorname{CMon}_{\infty}(\mathcal{D})\right) \simeq \lim _{k} \operatorname{Fun}^{k \text {-fin }}\left(\mathcal{C}, \operatorname{CMon}_{\infty}(\mathcal{D})\right) \simeq \\
& \varliminf_{k} \lim _{\ell} \operatorname{Fun}^{k \text {-fin }}\left(\mathcal{C}, \operatorname{CMon}_{\ell}(\mathcal{D})\right) \simeq \lim _{k} \operatorname{Fun}^{k \text {-fin }}\left(\mathcal{C}, \operatorname{CMon}_{k}(\mathcal{D})\right) \simeq \\
& \lim _{k} \operatorname{Fun}^{k \text {-fin }}(\mathcal{C}, \mathcal{D}) \simeq \operatorname{Fun}^{\infty-\text { fin }}(\mathcal{C}, \mathcal{D}) \text {. }
\end{aligned}
$$

As a corollary, for every $m$-semiadditive $\infty$-category we have a unique lift of the Yoneda embedding to a $\mathrm{CMon}_{m}$-enriched Yoneda embedding.

Corollary 2.1.17. Let $-2 \leq m \leq \infty$. For every $\mathcal{C} \in$ Cat $^{\oplus-m}$, there is a unique fully faithful m-semiadditive functor

$$
\left\llcorner^{\mathrm{CMon}_{m}}: \mathcal{C} \hookrightarrow \operatorname{Fun}\left(\mathcal{C}^{\mathrm{op}}, \mathrm{CMon}_{m}\right)\right.
$$

whose composition with the forgetful functor $\mathrm{CMon}_{m} \rightarrow \mathcal{S}$ is the Yoneda embedding. 
Proof. Taking $\mathcal{D}=\mathcal{S}$ in Proposition 2.1.16, shows that the ordinary Yoneda embedding

$$
\text { よ: } \mathcal{C} \hookrightarrow \operatorname{Fun}^{m-\operatorname{fin}}\left(\mathcal{C}^{o p}, \mathcal{S}\right) \subseteq \operatorname{Fun}\left(\mathcal{C}^{o p}, \mathcal{S}\right)
$$

lifts uniquely to a fully faithful $m$-finite limit preserving functor

$$
\left\llcorner^{\mathrm{CMon}_{m}}: \mathcal{C} \hookrightarrow \operatorname{Fun}^{m-\mathrm{fin}}\left(\mathcal{C}^{o p}, \mathrm{CMon}_{m}\right) \subseteq \operatorname{Fun}\left(\mathcal{C}^{o p}, \mathrm{CMon}_{m}\right) .\right.
$$

The $\mathrm{CMon}_{m}$-enriched Yoneda embedding $\left\llcorner^{\mathrm{CMon}_{m}}\right.$ corresponds to a functor

$$
\operatorname{hom}^{\mathrm{CMon}_{m}}(-,-): \mathcal{C} \times \mathcal{C}^{o p} \rightarrow \mathrm{CMon}_{m}
$$

whose composition with the forgetful functor $\mathrm{CMon}_{m} \rightarrow \mathcal{S}$ is the functor $\operatorname{Map}_{\mathcal{C}}(-,-)$. Thus, we obtain a canonical structure of an $m$-commutative monoid on each mapping space in $\mathcal{C}$. Informally, the "wrong way" maps for $A \stackrel{q}{\rightarrow} B$, in the higher commutative monoid structure on $\operatorname{Map}_{\mathcal{C}}(X, Y)$, are given by integration

$$
\int_{q}: \operatorname{Map}_{\mathcal{C}}(X, Y)^{A} \rightarrow \operatorname{Map}_{\mathcal{C}}(X, Y)^{B}
$$

Remark 2.1.18. It is overwhelmingly likely that an $m$-semiadditive $\infty$-category $\mathcal{C}$ can be canonically enriched in $\mathrm{CMon}_{m}$ (for e.g. in the sense of [GH15] or [Hin16]), such that the $\mathrm{CMon}_{m}$-valued mapping objects coincide with our definition above. In case $\mathcal{C}$ is further assumed to be presentable, this follows from the fact that $\mathcal{C}$ is left tensored over $\mathrm{CMon}_{m}$ (see [Har17, Lemma 5.20], Proposition 5.3.1 and [Lura, Proposition 4.2.1.33]).

\section{$2.2 \quad$ Examples}

We now review some examples of $m$-semiadditive $\infty$-categories and the behavior of cardinalities of $m$-finite spaces in them.

\section{Universal}

It is proved in [Har17], that the following is the universal example of an $m$-semiadditive $\infty$-category. In particular, it shows that in general, the operations $|A|$ need not reduce to something "classical":

Example 2.2.1 (Universal case). By [Har17, Corollary 5.7], for $-2 \leq m<\infty$ the symmetric monoidal $\infty$-category of $\operatorname{spans} \mathcal{C}=\operatorname{Span}\left(\mathcal{S}_{m \text {-fin }}\right)$ is the universal $m$-semiadditive $\infty$-category. For every $A \in \mathcal{S}_{m \text {-fin }}$, we have

$$
|A|_{\mathrm{pt}}=(\mathrm{pt} \leftarrow A \rightarrow \mathrm{pt}) \quad \in \quad \pi_{0} \operatorname{Map}_{\mathrm{Span}\left(\mathcal{S}_{m-\mathrm{fin}}\right)}(\mathrm{pt}, \mathrm{pt}) .
$$

Moreover, $\pi_{0} \operatorname{Map}_{\mathrm{Span}\left(\mathcal{S}_{m \text {-fin }}\right)}(\mathrm{pt}, \mathrm{pt})$ is the set of isomorphism classes of $m$-finite spaces with the semiring structure given by (see Remark 2.1.10):

$$
|A|+|B|=|A \sqcup B|, \quad|A| \cdot|B|=|A \times B| .
$$


Informally, the universality of Example 2.2.1 is reflected in its construction as follows. The collection of spaces $\mathcal{S}_{m \text {-fin }} \subseteq \mathcal{S}$ is generated under $m$-finite colimits from the point pt $\in \mathcal{S}$. The "right way" maps in $\operatorname{Span}\left(\mathcal{S}_{m \text {-fin }}\right)$ encode the usual covariant functoriality of these colimits. The "wrong way" maps in $\operatorname{Span}\left(\mathcal{S}_{m \text {-fin }}\right)$ encode the contravariant functoriality arising from "integration along the fibers".

A closely related example is the $\infty$-category $\mathrm{CMon}_{m}$ of $m$-commutative monoids, which is shown in [Har17, Corollary 5.19, Corollary 5.21], to be the universal presentable $m$-semiadditive $\infty$-category. The Yoneda embedding induces a fully faithful $m$-semiadditive (symmetric monoidal) functor

$$
\operatorname{Span}\left(\mathcal{S}_{m-\text { fin }}\right) \hookrightarrow \mathrm{CMon}_{m},
$$

taking each $m$-finite space $A$ to the "free $m$-commutative monoid" on $A$. From this we get that cardinalities in $\mathrm{CMon}_{m}$ are computed essentially in the same way as in $\operatorname{Span}\left(\mathcal{S}_{m \text {-fin }}\right)^{7}$. In section 5, we shall discuss more systematically the universality of $\mathrm{CMon}_{m}$ (see Proposition 5.3.1).

\section{Rational}

There are however some situations in which the operations $|A|$ can be expressed in terms of classical invariants.

Example 2.2.2 (Homotopy cardinality). For a $\pi$-finite space $A$, Baez and Dolan [Bae] define the homotopy cardinality of $A$ to be the following non-negative rational number

$$
|A|_{0}:=\sum_{a \in \pi_{0}(A)} \prod_{n \geq 1}\left|\pi_{n}(A, a)\right|^{(-1)^{n}} \in \mathbb{Q} \geq 0 .
$$

This notion can be seen as a special case of the cardinality of a $\pi$-finite space in a higher semiadditive $\infty$-category as follows. We say that an $\infty$-category $\mathcal{C}$ is semirational if it is 0 -semiadditive and for each $n \in \mathbb{N}$, multiplication by $n$ is invertible in $\mathcal{C}\left(\right.$ e.g. $\mathcal{C}=\mathrm{Sp}_{\mathbb{Q}}$ or $\left.\operatorname{Vec}_{\mathbb{Q}}\right)$. We shall see that a semirational $\infty$-category which admits all 1 -finite colimits is automatically $\infty$-semiadditive and for every $\pi$-finite space $A$, we have

$$
|A|_{\mathcal{C}}=|A|_{0} \quad \in \quad \mathbb{Q}_{\geq 0} \subseteq \operatorname{End}\left(\operatorname{Id}_{\mathcal{C}}\right)
$$

We note that formula (1) is completely determined by the additivity of the cardinality under coproducts and the following "multiplicativity property": For every fiber sequence of $\pi$-finite spaces

$$
F \rightarrow A \rightarrow B
$$

such that $B$ is connected, we have $|A|=|F||B|^{8}$.

Remark 2.2.3. In fact, we shall prove in Proposition 2.3 .4 a somewhat sharper result. Let $\mathcal{C}$ be a 0 -semiadditive $\infty$-category, which admits $\pi$-finite colimits, and let $A$ be a $\pi$-finite space. If $A$ satisfies the following condition:

\footnotetext{
${ }^{7}$ The relation between $\operatorname{Span}\left(\mathcal{S}_{m \text {-fin }}\right)$ and $\mathrm{CMon}_{m}$ is somewhat analogues to the relation between the $\infty$-category $\mathrm{Sp}^{\omega}$ of finite spectra and the $\infty$-category Sp of all spectra.

${ }^{8}$ This follows from the long exact sequence in homotopy groups, and is reminiscent of the "additivity property" of the Euler characteristic.
} 
(*) The orders of the homotopy groups of $A$ are invertible on all objects of $\mathcal{C}$.

Then $A$ is $\mathcal{C}$-ambidextrous and $|A|_{\mathcal{C}}=|A|_{0}$.

From the perspective of the theory we are about to develop, semirational $\infty$-categories are 0 semiadditive $\infty$-categories of "height 0 ". One of our goals is to generalize the above phenomena to "higher heights" (see Proposition 3.2.2 and Proposition 3.2.5).

\section{Chromatic}

Examples of $\infty$-semiadditive $\infty$-categories of "higher height" appear naturally in chromatic homotopy theory. For a given prime $p$ and $0 \leq n<\infty$, let $K(n)$ be the Morava $K$-theory spectrum of height $n$ at the prime $p$. One of the main results of [HL13] is that the localizations $\operatorname{Sp}_{K(n)}$ are $\infty$-semiadditive. In particular, we can consider $K(n)$-local cardinalities of $\pi$-finite spaces. For $n=0$, we have $\mathrm{Sp}_{K(n)} \simeq \mathrm{Sp}_{\mathbb{Q}}$ and we recover the homotopy cardinality (Example 2.2.2). Similarly, since $\operatorname{Sp}_{K(n)}$ is $p$-local for all $n$, for every $\pi$-finite space $A$ whose homotopy groups have cardinality prime to $p$, the $K(n)$-local cardinality of $A$ coincides with its homotopy cardinality for all $n$ (see Remark 2.2.3). In particular, it is independent of $n$. However, for $n \geq 1$ the prime $p$ is not invertible in $\operatorname{Sp}_{K(n)}$. Thus, there are $\pi$-finite spaces $A$ (e.g. $\pi$-finite $p$-spaces), which are ambidextrous even though they do not satisfy condition $(*)$ of Remark 2.2.3. For such spaces $A$, the $K(n)$-local cardinality does depend on $n$ and in general does not (and can not) agree with the homotopy cardinality. ${ }^{9}$

To study the $K(n)$-local cardinalities of $\pi$-finite spaces, it is useful to consider their image in Morava $E$-theory. For every integer $n \geq 1$, we let $E_{n}$ be the Morava $E$-theory associated with some formal group of height $n$ over $\overline{\mathbb{F}}_{p}$, viewed as an object of $\mathrm{CAlg}\left(\mathrm{Sp}_{K(n)}\right)$. In particular, we have a (non-canonical) isomorphism

$$
\pi_{*} E_{n} \simeq \mathbb{W}\left(\overline{\mathbb{F}}_{p}\right)\left[\left[u_{1}, \ldots, u_{n-1}\right]\right]\left[u^{ \pm 1}\right] \quad\left|u_{i}\right|=0, \quad|u|=2 .
$$

Example 2.2.4 (Chromatic cardinality). The $\infty$-category $\Theta_{n}=\operatorname{Mod}_{E_{n}}\left(\operatorname{Sp}_{K(n)}\right)$ is $\infty$-semiadditive [CSY18, Theorem 5.3.1] and hence one can consider cardinalities of $\pi$-finite spaces in $\pi_{0} E_{n}$. We define the ( $p$-typical) height $n$ cardinality of $A$ to be

$$
|A|_{n}:=|A|_{\Theta_{n}} \quad \in \quad \pi_{0} E_{n} .
$$

It makes sense to consider $\overline{\mathbb{Q}}$ as $E_{0}$, in which case we recover the homotopy cardinality (Example 2.2.2). The technology of [Lur19] allows one to derive a rather explicit formula for $|A|_{n}$, for heights $n>0$ as well. Let $\widehat{L}_{p} A:=\operatorname{Map}\left(B \mathbb{Z}_{p}, A\right)$ be the $p$-adic free loop space of $A$. One can show that the element $|A|_{n} \in \pi_{0} E_{n}$ belongs to the subring $\mathbb{Z}_{(p)} \subseteq \pi_{0} E_{n}$ and satisfies $|A|_{n}=\left|\widehat{L}_{p} A\right|_{n-1}$. Applying this relation inductively we obtain the formula

$$
|A|_{n}=\left|\operatorname{Map}\left(B \mathbb{Z}_{p}^{n}, A\right)\right|_{0} \quad \in \quad \mathbb{Z}_{(p)} .
$$

If $A$ happens to be a $p$-space, then $\widehat{L}_{p} A$ coincides with the ordinary free loop space $L A:=$ $\operatorname{Map}\left(S^{1}, A\right)$. Thus, $|A|_{n}$ can be computed as the homotopy cardinality of the space of maps from the $n$-dimensional torus to $A$.

\footnotetext{
${ }^{9}$ Note that the rationalization functor $L_{\mathbb{Q}}: \mathrm{Sp}_{K(n)} \rightarrow \mathrm{Sp}_{\mathbb{Q}}$ does not preserve colimits in general and so does not preserve cardinalities. It does however preserve colimits which are indexed on $\pi$-finite spaces whose homotopy groups have order prime to $p$.
} 
We shall not get here into the details of how formula (2) is deduced from the results of [Lur19], as we shall only need the following special case:

Proposition 2.2.5. For all $k, n \geq 0$ we have $\left|B^{k} C_{p}\right|_{n}=p^{\left(\begin{array}{c}n-1 \\ k\end{array}\right)} \cdot{ }^{10}$

This was proved independently in [CSY18, Lemma 5.3.3] by relating the cardinality to the symmetric monoidal dimension. However, we shall use the general formula (2) in some examples to illustrate interesting phenomena.

While the structure of the rings $\pi_{0} \mathbb{S}_{K(n)}$ is not entirely understood in general, it follows from [BG18] and [BGH17] that:

Proposition 2.2.6. For all $p$ and $n$, the image of the unit map $\pi_{0} \mathbb{S}_{K(n)} \stackrel{u}{\rightarrow} \pi_{0} E_{n}$ is $\mathbb{Z}_{p} \subseteq \pi_{0} E_{n}$ and the kernel is precisely the nil-radical.

Proof. Let $\Gamma_{n}$ be the Morava stabilizer group associated with $E_{n}$. We have an action of $\Gamma_{n}$ on $E_{n}$ by commutative algebra maps and thus, the map $u$ factors through the fixed points $\left(\pi_{0} E_{n}\right)^{\Gamma_{n}} \subseteq \pi_{0} E_{n}$. By [BG18, Lemma 1.33], we have

$$
\left(\pi_{0} E_{n}\right)^{\Gamma_{n}}=H_{c}^{0}\left(\Gamma ; \pi_{0} E_{n}\right)=\mathbb{Z}_{p} \subseteq \pi_{0} E_{n} .
$$

By [BGH17, Theorem 2.3.5], the $E_{\infty}$-page of the descent spectral sequence

$$
H_{c}^{s}\left(\Gamma ;\left(E_{n}\right)_{t}\right) \Longrightarrow \pi_{t-s}\left(\mathbb{S}_{K(n)}\right)
$$

has a horizontal vanishing line. Since the spectral sequence is multiplicative, this implies that all elements in $\pi_{0} \mathbb{S}_{K(n)}$ with positive filtration degree are nilpotent. Finally, since $\mathbb{S}_{K(n)}$ admits a ring map from the $p$-complete sphere, the map $\pi_{0} \mathbb{S}_{K(n)} \stackrel{u}{\rightarrow} \mathbb{Z}_{p}$ is surjective.

Thus, for every $\pi$-finite space $A$, the identity

$$
|A|_{\operatorname{Sp}_{K(n)}}=|A|_{n}
$$

holds up to nilpotents. We do not know, however, whether it holds in $\pi_{0} \mathbb{S}_{K(n)}$.

\section{Categorical}

Another family of examples of higher semiadditive $\infty$-categories arises from category theory itself.

Proposition 2.2.7. For every $-2 \leq m \leq \infty$ the $\infty$-category Cat $_{m \text {-fin }}$ is m-semiadditive.

Proof. The case $m<\infty$ is exactly [Har17, Proposition 5.26]. We now wish to show that Cat $\infty$-fin is $k$-semiadditive for every $k<\infty$. By [Lura, Remark 4.8.1.6], both Cat $_{k \text {-fin }}$ and Cat C-fin $_{\text {admit }}$ closed symmetric monoidal structures, and by [Lura, Proposition 4.8.1.3], there exists a symmetric monoidal functor $\mathcal{P}: \mathrm{Cat}_{k \text {-fin }} \rightarrow \mathrm{Cat}_{\infty \text {-fin. }}$ By [Lura, Remark 4.8.1.8] and [Lur09, Proposition 5.3.6.2(2)], $\mathcal{P}$ admits a right adjoint and thus preserves colimits. Hence, $\mathrm{Cat}_{\infty}$-fin is $k$-semiadditive by [CSY18, Corollary 3.3.2(2)].

${ }^{10}$ For height $n=0$ this should be interpreted via the identity $\left(\begin{array}{c}-1 \\ k\end{array}\right)=(-1)^{k}$. 
Example 2.2.8 (Categorical cardinality). Let $-2 \leq m \leq \infty$ and let $\mathcal{C} \in \mathrm{Cat}_{m \text {-fin. }}$. For every $m$-finite space $A$ the $m$-semiadditive structure of Cat ${ }_{m \text {-fin }}$ gives rise to a functor $|A|_{\mathcal{C}}: \mathcal{C} \rightarrow \mathcal{C}$. When $m<\infty$ it is shown in [Har17, Section 5.2] that $|A|_{\mathcal{C}}$ is given by taking the constant colimit on $A$. That is, it takes an object $X \in \mathcal{C}$ to the object $X[A] \in \mathcal{C}$. Since the forgetful functor $\mathrm{Cat}_{\infty \text {-fin }} \rightarrow \mathrm{Cat}_{m \text {-fin }}$ preserves limits, and hence m-semiadditive, the same holds for $m=\infty$. This is very suggestive of the idea that "multiplication by $|A|$ on $\mathcal{C}$ " is given by "summing each object $X \in \mathcal{C}$ with itself $A$ times". A closely related example is discussed in [HL13, Example 4.3.11], where it is shown that $\operatorname{Pr}$ is $\infty$-semiadditive (in fact, every $m$-truncated space, not necessarily $\pi$-finite, is Pr-ambidextrous).

Remark 2.2.9. There is a different approach to the higher semiadditivity of $\mathrm{Cat}_{m \text {-fin }}$, based on the notion of ambidextrous adjunctions of $(\infty, 2)$-categories. We sketch the argument to demonstrate the role of higher categorical structures as a useful perspective on ambidexterity phenomena. Given an $m$-finite space $A$, the adjunction

$$
A^{*}: \mathrm{Cat}_{m \text {-fin }} \leftrightarrows \mathrm{Cat}_{m \text {-fin }}^{A}: A_{*}
$$

can be naturally promoted to an adjunction of $(\infty, 2)$-categories. Moreover, the unit $u$ and counit $c$ of $A^{*} \dashv A_{*}$, as 1-morphisms in the respective $(\infty, 2)$-categories of endofunctors, can be shown to have left adjoints $u^{L}$ and $c^{L}$ respectively. Thus, we are in a situation which is dual to the notion of an ambidextrous adjunction of [HSSS18, Definition 2.1]. It follows by an elementary argument that $u^{L}$ and $c^{L}$ exhibit $A_{*}$ as a left adjoint of $A^{*}$ (see [HSSS18, Remark 2.2]). Hence, by Proposition 2.1.3,

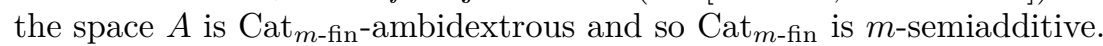

The functor that takes an $\infty$-category to its opposite induces an equivalence $\mathrm{Cat}_{m \text {-fin }} \simeq \mathrm{Cat}^{m \text {-fin }}$. Hence, the $\infty$-category $\mathrm{Cat}^{m \text {-fin }}$ is $m$-semiadditive as well and the higher semiadditive structure is given by taking limits. For every $\infty$-category $\mathcal{C}$ with finite (co)products, the (co)product endows the space of objects $\mathcal{C}^{\simeq}$ with a (co)Cartesian commutative monoid structure. Using the $m$-semiadditivity of $\mathrm{Cat}_{m \text {-fin }}$ and $\mathrm{Cat}^{m \text {-fin }}$ together with the $\mathrm{CMon}_{m}$-enriched Yoneda embedding provided by Corollary 2.1.17, this too can be generalized to all $0 \leq m \leq \infty$. Given $\mathcal{C} \in \mathrm{Cat}_{m \text {-fin }}$, by the $m$-semiadditivity of $\mathrm{Cat}_{m \text {-fin }}$, the mapping space $\operatorname{Map}_{m \text {-fin }}\left(\mathcal{S}_{m \text {-fin }}, \mathcal{C}\right)$ admits a canonical structure of an $m$-commutative monoid. On the other hand, since $\mathcal{S}_{m \text {-fin }}$ is freely generated from a point under $m$-finite colimits [Lura, Notation 4.8.5.2], we have

$$
\operatorname{Map}_{m \text {-fin }}\left(\mathcal{S}_{m \text {-fin }}, \mathcal{C}\right) \simeq \operatorname{Map}(\mathrm{pt}, \mathcal{C}) \simeq \mathcal{C}^{\simeq}
$$

Definition 2.2.10. For $\mathcal{C} \in \mathrm{Cat}_{m \text {-fin }}$, we refer to the above $m$-commutative monoid structure on the space of objects $\mathcal{C} \simeq$ as the coCartesian structure. A completely analogous construction endows the space of objects of each $\mathcal{C} \in \mathrm{Cat}^{m \text {-fin }}$ with a Cartesian $m$-commutative monoid structure.

As explained in [Har17, Section 5.2], the integration operations for the (co)Cartesian $m$-commutative monoid structure on $\mathcal{C}^{\simeq}$ are given by taking $m$-finite (co)limits. Finally, the $\infty$-category $\mathrm{Cat}^{\oplus-m}$ of $m$-semiadditive $\infty$-categories and $m$-semiadditive functors is a full subcategory of both $\mathrm{Cat}_{m \text {-fin }}$ and $\mathrm{Cat}^{\mathrm{m} \text {-fin }}$. Moreover, we have the following:

Proposition 2.2.11. Let $-2 \leq m \leq \infty$. The full subcategory Cat $^{\oplus-m} \subseteq$ Cat $_{m \text {-fin }}$ (resp. Cat $^{m \text {-fin }}$ ) is closed under colimits and in particular is m-semiadditive. 
Proof. The functor $(-)^{\mathrm{op}}: \mathrm{Cat}_{m \text {-fin }} \rightarrow \mathrm{Cat}^{m \text {-fin }}$ that takes an $\infty$-category to its opposite is an equivalence. By Proposition 2.1.4, $(-)^{\mathrm{op}}$ restricts to an involution of Cat ${ }^{\oplus-m}$. It thus suffices to consider only the inclusion $\iota$ : $\mathrm{Cat}^{\oplus-m} \hookrightarrow \mathrm{Cat}^{m \text {-fin }}$. By Proposition 2.1.16, $\iota$ admits a right adjoint given by $\mathrm{CMon}_{m}(-)$ and thus preserve colimits.

\subsection{Amenability}

Let $A \stackrel{q}{\rightarrow} B$ be a $\mathcal{C}$-ambidextrous map. Recall from [CSY18, Definition 3.1.7], that $q$ is called $\mathcal{C}$ amenable if $|q|_{\mathcal{C}}$ is invertible. As with ambidexterity, amenability is a fiber-wise property ([CSY18, Corollary 3.1.16]). Thus, we shall be mainly interested in $\mathcal{C}$-amenable spaces. i.e. those whose $\mathcal{C}$ cardinality is invertible.

Remark 2.3.1. We warn the reader not to confuse the condition that the natural transformation $|q|_{\mathcal{C}}$ is invertible with the condition that the natural transformation $\mathrm{Nm}_{q}$ is invertible, which is equivalent to $q$ being $\mathcal{C}$-ambidextrous (which is itself a prerequisite for defining $|q|_{\mathcal{C}}$ ).

In this section, we extend some results from [CSY18] regarding amenability. The main point is that while $A$-ambidexterity allows us to sum over $A$-families of maps, $A$-amenability allows us to average over $A$-families of maps, which in turn facilitates "transfer arguments" along $A$. We shall explore how this condition affects the higher semiadditive structure.

\section{Closure properties}

For a map of spaces, the condition of $\mathcal{C}$-amenability, as the condition of $\mathcal{C}$-ambidexterity, is fiberwise. However, unlike $\mathcal{C}$-ambidextrous maps, $\mathcal{C}$-amenable maps are not closed under composition. To understand the situation, it suffices to consider the case $A \stackrel{q}{\rightarrow} B \rightarrow$ pt. By the additivity of cardinality (Proposition 2.1.9), we get $|A|=\int_{B}|q|$. Assume for simplicity that $B$ is connected and that the fiber of $q$ is $F$. The transformation $|q|$ equals $|F|$ at each point $b \in B$. Thus, it is tempting to presume that $|A|=|B| \cdot|F|$ and hence if both $|B|$ and $|F|$ are invertible, then so is $|A|$. However, for this reasoning to hold we need to know that $|q|$ is constant on $B$ with value $|F|$. Alas, in general $|q|$ is not constant, even when $|F|$ is invertible, and $|A|$ need not equal $|B| \cdot|F|$ (see Example 2.3.6). On the positive side, we show that $|q|$ must be constant if we require in addition to the invertibility of $|F|$ that $F \rightarrow A \stackrel{q}{\rightarrow} B$ is a principal fiber sequence.

Definition 2.3.2. We call a map $A \stackrel{q}{\rightarrow} B$ of spaces principal if it can be extended to a fiber sequence $A \stackrel{q}{\rightarrow} B \stackrel{f}{\rightarrow} E$.

We note that for a principal map all the fibers are isomorphic even if the target is not connected.

Proposition 2.3.3. Let $\mathcal{C} \in \mathrm{Cat}_{\infty}$ and let $A \stackrel{q}{\rightarrow} B$ be a principal $\mathcal{C}$-ambidextrous map of $\mathcal{C}$ ambidextrous spaces with fiber $F$. For $X \in \mathcal{C}$, if $|F|_{X}$ is invertible, then

$$
|q|_{B^{*} X}=|F|_{B^{*} X} \quad \in \quad \operatorname{End}\left(B^{*} X\right)
$$

and

$$
|A|_{X}=|F|_{X}|B|_{X} .
$$


Proof. The base change of $q$ along itself is a map $A \times_{B} A \stackrel{\tilde{q}}{\rightarrow} A$, which is a principal map with a section. Therefore $\widetilde{q}$ is isomorphic to the projection $F \times A \stackrel{\pi_{A}}{\longrightarrow} A$. Hence, $q \times_{B} q \simeq q \times_{B} \pi_{B}$, where $F \times B \stackrel{\pi_{B}}{\longrightarrow} B$ is the projection. We get from [CSY18, Corollary 3.1.14] that

$$
|q|^{2}=\left|q \times_{B} q\right|=\left|q \times_{B} \pi_{B}\right|=|q|\left|\pi_{B}\right| \in \operatorname{End}\left(\operatorname{Id}_{\mathcal{C}^{B}}\right) .
$$

If $|F|_{X}$ is invertible, then $|q|_{B^{*} X}$ is invertible, and thus by the above, we get ([CSY18, Proposition $3.1 .13])$

$$
|q|_{B^{*} X}=\left|\pi_{B}\right|_{B^{*} X}=B^{*}\left(|F|_{X}\right)=|F|_{B^{*} X} \quad \in \quad \operatorname{End}\left(B^{*} X\right) .
$$

We can now integrate along $B$ and get

$$
|A|_{X}=\int_{B}|q|_{B^{*} X}=\int_{B} B^{*}\left(|F|_{X}\right)=|F|_{X}|B|_{X} \in \operatorname{End}(X) .
$$

As a simple application, we deduce that when the homotopy groups of a $\pi$-finite space have invertible cardinality in $\mathcal{C}$, the notion of cardinality degenerates to the homotopy cardinality (Remark 2.2.3).

Proposition 2.3.4. Let $\mathcal{C} \in \mathrm{Cat}^{\oplus-0}$ and let $A$ be a $\pi$-finite space. Assume that $\mathcal{C}$ admits $A$-colimits and the order of each homotopy group of $A$ is invertible in $\operatorname{End}\left(\operatorname{Id}_{\mathcal{C}}\right)$. Then, $A$ is $\mathcal{C}$-ambidextrous and $|A|_{\mathcal{C}}=|A|_{0}$. In particular, if $A$ is connected, then it is $\mathcal{C}$-amenable.

Proof. By Remark 2.1.10, we may assume that $A$ is connected. We prove the claim for all connected $m$-finite $A$ by induction on $m \geq 0$, where the case $m=0$ is trivial. We prove the claim for some $m>0$, assuming it holds for $m-1$. Choose a base point in $A$ and consider the connected component $(\Omega A)^{\circ} \subseteq \Omega A$ of the identity loop. The space $(\Omega A)^{\circ}$ satisfies the assumptions of the inductive hypothesis. Hence, $(\Omega A)^{\circ}$ is amenable and $\left|(\Omega A)^{\circ}\right|_{\mathcal{C}}=\left|(\Omega A)^{\circ}\right|_{0}$. Now, $\Omega A$ is just the coproduct of $\left|\pi_{1} A\right|$ copies of $(\Omega A)^{\circ}$. Hence, by Remark 2.1.10 and the inductive hypothesis, we have

$$
|\Omega A|_{\mathcal{C}}=\left|\pi_{1} A\right| \cdot\left|(\Omega A)^{\circ}\right|_{\mathcal{C}}=\left|\pi_{1} A\right| \cdot\left|(\Omega A)^{\circ}\right|_{0}=|\Omega A|_{0} .
$$

Moreover, $|\Omega A|_{\mathcal{C}}$ is invertible as $\left|\pi_{1} A\right|$ is invertible by assumption and $\left|(\Omega A)^{\circ}\right|_{\mathcal{C}}$ is invertible by the inductive hypothesis. That is, $\Omega A$ is $\mathcal{C}$-amenable. Finally, consider the principal fiber sequence

$$
\Omega A \rightarrow \mathrm{pt} \rightarrow A \text {. }
$$

Since $\Omega A$ is $\mathcal{C}$-amenable, by [CSY18, Proposition 3.1.17], the space $A$ is $\mathcal{C}$-ambidextrous and by Proposition 2.3.3, we have

$$
|A|_{\mathcal{C}}=|\Omega A|_{\mathcal{C}}^{-1}=|\Omega A|_{0}^{-1}=|A|_{0} .
$$

From Proposition 2.3.3, we also deduce that $\mathcal{C}$-amenable maps are partially closed under composition:

Corollary 2.3.5. Let $\mathcal{C} \in \mathrm{Cat}_{\infty}$ and let $A \stackrel{f}{\rightarrow} B \stackrel{g}{\rightarrow} C$ be a pair of composable maps of spaces. If $f$ and $g$ are $\mathcal{C}$-amenable and $f$ is principal, then $g \circ f$ is $\mathcal{C}$-amenable.

Proof. We can check that $g \circ f$ is $\mathcal{C}$-amenable, by pulling back along pt $\rightarrow C$ for every point of $C$. In other words, we can assume that $C=$ pt. Taking $F$ to be the fiber of $f$, we have a principal fiber sequence $F \rightarrow A \rightarrow B$ where $F$ and $B$ are $\mathcal{C}$-amenable; thus the result follows from Proposition 2.3.3. 


\section{Counter examples}

We conclude this subsection with a discussion of the necessity of the conditions in Proposition 2.3.3. For starters, if $|F|$ is not invertible, then the identity $|A|=|F||B|$ is (very much) false in general. The following examples show that the condition on the fiber sequence to be principal can also not be dropped. The first example shows that $\mathcal{C}$-cardinality need not be multiplicative even when the fiber and base space are $\mathcal{C}$-amenable. Moreover, in such case, the total space need not even be $\mathcal{C}$-amenable and so in particular, $\mathcal{C}$-amenable maps are not closed under composition.

Example 2.3.6. Let $p$ be an odd prime and let $\Theta_{n}:=\operatorname{Mod}_{E_{n}}\left(\operatorname{Sp}_{K(n)}\right)$. We consider the map $B^{2} C_{p} \stackrel{f}{\rightarrow} B^{4} C_{p}$ classifying the cup-square operation $x \mapsto x \cup x$ on mod $p$ cohomology, and the associated fiber sequence

$$
F \rightarrow B^{2} C_{p} \stackrel{f}{\rightarrow} B^{4} C_{p}
$$

The only non-trivial homotopy groups of $F$ are $\pi_{2} F \simeq \pi_{3} F \simeq C_{p}$, but the Postnikov invariant represented by $f$ in $H^{4}\left(B^{2} C_{p} ; C_{p}\right)$ is non-zero. Using Example 2.2.4, we have $|F|_{n}=\left|L^{n} F\right|_{0}$ and we can compute it using the fiber sequence

$$
L^{n} F \rightarrow L^{n} B^{2} C_{p} \rightarrow L^{n} B^{4} C_{p},
$$

via the induced long exact sequence on homotopy groups. The only complication arises at the level of $\pi_{0}$, where we need to compute the size of the kernel of the cup-square map

$$
\pi_{0} L^{n} B^{2} C_{p}=H^{2}\left(T^{n} ; C_{p}\right) \rightarrow H^{4}\left(T^{n} ; C_{p}\right)=\pi_{0} L^{n} B^{4} C_{p} .
$$

Namely, the number of $n$-dimensional 2 -forms over $C_{p}$ that square to zero. Since this is the number of 2-forms of rank lower or equal 1, one can write down an explicit combinatorial formula for it. This leads to the following explicit formula:

$$
|F|_{n}=p^{\left(\begin{array}{c}
n-1 \\
3
\end{array}\right)} \cdot \frac{p^{3-n}+p^{n}-p-1}{p^{2}-1} .
$$

In particular, taking $n=4$, we get $|F|_{4}=p^{3}+p-1$, which is an invertible element in $\pi_{0} E_{4}$. It follows that $F$ is $\Theta_{4}$-amenable. Nevertheless,

$$
|F|_{4}\left|B^{4} C_{p}\right|_{4}=p^{3}+p-1
$$

which differs from

$$
\left|B^{2} C_{p}\right|_{4}=p^{\left(\begin{array}{l}
3 \\
2
\end{array}\right)}=p^{3}
$$

Moreover, $F$ and $B^{4} C_{p}$ are both $\Theta_{4}$-amenable, but $B^{2} C_{p}$ is not. Thus, the maps $B^{2} C_{p} \stackrel{f}{\rightarrow} B^{4} C_{p}$ and $B^{4} C_{p} \rightarrow$ pt are $\Theta_{4}$-amenable, but their composition is not.

The next example shows that $\mathcal{C}$-cardinality need not be multiplicative when the fiber and total space are $\mathcal{C}$-amenable. Moreover, the base space in this case need not be $\mathcal{C}$-amenable and so in particular the class of $\mathcal{C}$-amenable maps does not satisfy "left cancellation" (compare [CSY18, Theorem 2.4.5]). 
Example 2.3.7. Let $p=2$ and $\Theta_{1}=\operatorname{Mod}_{E_{1}}\left(\operatorname{Sp}_{K(1)}\right)$. Consider the (non-principal) fiber sequence

$$
\Sigma_{3} / C_{2} \rightarrow B C_{2} \rightarrow B \Sigma_{3}
$$

It can be shown using Example 2.2.4, that for $p=2$ we have

$$
\left|B \Sigma_{3}\right|_{1}=\left|\widehat{L}_{p} B \Sigma_{3}\right|_{0}=\frac{2}{3} .
$$

In particular,

$$
\left|\Sigma_{3} / C_{2}\right|\left|B \Sigma_{3}\right|=2 \neq 1=\left|B C_{2}\right|_{1} .
$$

Moreover, $\Sigma_{3} / C_{2}$ and $B C_{2}$ are $\Theta_{1}$-amenable, but $B \Sigma_{3}$ is not. Thus, the map $B C_{2} \rightarrow B \Sigma_{3}$ and the composition $B C_{2} \rightarrow B \Sigma_{3} \rightarrow$ pt are $\Theta_{1}$-amenable, but $B \Sigma_{3} \rightarrow$ pt is not.

\subsection{Acyclic Maps}

In this subsection, we show that the amenability of a loop-space is equivalent to the "triviality" of limits and colimits over its classifying space. This characterization is interesting in that it does not directly involve the higher semiadditive structure.

\section{Definitions \& basic properties}

We begin by introducing the notions of "acyclicity" and "triviality", which are not immediately related to the theory of ambidexterity:

Definition 2.4.1. Let $A \stackrel{q}{\rightarrow} B$ be a map of spaces and let $\mathcal{C}$ be an $\infty$-category. We say that $q$ is $\mathcal{C}$-acyclic (resp. $\mathcal{C}$-trivial) if $\mathcal{C}$ admits all $q$-limits and $q$-colimits and $q^{*}: \mathcal{C}^{B} \rightarrow \mathcal{C}^{A}$ is fully faithful (resp. an equivalence).

It is a standard fact about adjoints, that $q^{*}$ is fully faithful, if and only if the counit $q ! q^{*} \stackrel{c_{!}^{q}}{\longrightarrow} \operatorname{Id}_{\mathcal{C}^{B}}$ is an isomorphism and if and only if the unit $\operatorname{Id}_{\mathcal{C}^{B}} \stackrel{u_{*}^{q}}{\longrightarrow} q_{*} q^{*}$ is an isomorphism. Similarly, $q^{*}$ is an equivalence, if furthermore $q^{*} q_{*} \stackrel{c_{*}^{q}}{\longrightarrow} \operatorname{Id}_{\mathcal{C}^{A}}$ is an isomorphism, or equivalently $\operatorname{Id}_{\mathcal{C}^{A}} \stackrel{u_{!}^{q}}{\longrightarrow} q^{*} q$ ! is an isomorphism. Like ambidexterity and amenability, acyclicity and triviality are fiber-wise conditions:

Proposition 2.4.2. Let $\mathcal{C} \in \mathrm{Cat}_{\infty} . A$ map of spaces $A \stackrel{q}{\rightarrow} B$ is $\mathcal{C}$-acyclic (resp. $\mathcal{C}$-trivial) if and only if each fiber of $q$ is $\mathcal{C}$-acyclic (resp. $\mathcal{C}$-trivial).

Proof. For each map $\widetilde{B} \stackrel{f}{\rightarrow} B$ we can form the following pullback square of spaces and the induced commutative square of $\infty$-categories
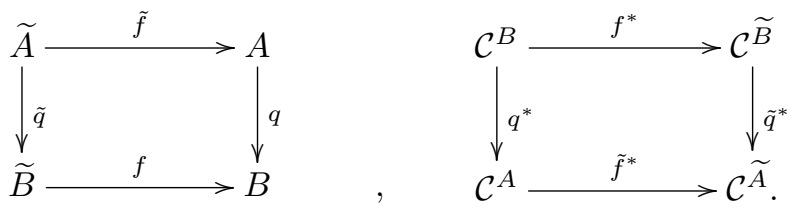
By [CSY18, Lemma 2.2.3], we have

$$
\begin{aligned}
& f^{*}\left(c_{!}^{q}\right)=c_{!}^{\tilde{q}} \in \operatorname{Map}\left(\tilde{q}_{!} \tilde{q}^{*}, \operatorname{Id}_{\mathcal{C}^{\tilde{B}}}\right) . \\
& \tilde{f}^{*}\left(u_{!}^{q}\right)=u_{!}^{\tilde{q}} \in \operatorname{Map}\left(\operatorname{Id}_{\mathcal{C}^{\tilde{A}}}, \tilde{q}^{*} \tilde{q}_{!}\right) .
\end{aligned}
$$

Thus, if $q$ is $\mathcal{C}$-acyclic (resp. $\mathcal{C}$-trivial), then $\tilde{q}$ is $\mathcal{C}$-acyclic (resp. $\mathcal{C}$-trivial). If $f$ (and hence also $\tilde{f}$ ) is surjective, then by the conservativity of $f^{*}$ (and $\tilde{f}^{*}$ ), the converse holds as well. Namely, if $\tilde{q}$ is $\mathcal{C}$-acyclic (resp. $\mathcal{C}$-trivial), then $q$ is $\mathcal{C}$-acyclic (resp. $\mathcal{C}$-trivial). Applying this to any section of $B \rightarrow \pi_{0} B=\widetilde{B}$ yields the claim.

The collections of $\mathcal{C}$-acyclic and $\mathcal{C}$-trivial spaces are also closed under extensions:

Corollary 2.4.3. Let $\mathcal{C} \in \mathrm{Cat}_{\infty}$ and let $A \stackrel{q}{\rightarrow} B$. If $B$ is $\mathcal{C}$-acyclic (resp. $\mathcal{C}$-trivial) and all the fibers of $q$ are $\mathcal{C}$-acyclic (resp. $\mathcal{C}$-trivial), then $A$ is $\mathcal{C}$-acyclic (resp. $\mathcal{C}$-trivial).

Proof. By Proposition 2.4.2, it suffices to show that $\mathcal{C}$-acyclic (resp. $\mathcal{C}$-trivial) maps are closed under composition, which is clear from the definition.

In presence of a compatible monoidal structure, one can check the acyclicity property on the unit:

Lemma 2.4.4. Let $A$ be a space and let $\mathcal{C} \in \operatorname{Alg}\left(\mathrm{Cat}_{\infty}\right)$ which is compatible with A-colimits. The space $A$ is $\mathcal{C}$-acyclic if and only if the fold map $\mathbb{1}[A] \stackrel{\nabla}{\rightarrow} \mathbb{1}$ is an isomorphism.

Proof. Assume $\mathbb{1}[A] \stackrel{\nabla}{\rightarrow} \mathbb{1}$ is an isomorphism. By assumption, for every $X \in \mathcal{C}$, tensoring the isomorphism $\mathbb{1}[A] \stackrel{\nabla}{\rightarrow} \mathbb{1}$ with $X$ gives the fold map $X[A] \stackrel{\nabla}{\rightarrow} X$. Hence, $A_{!} A^{*} X=X[A] \stackrel{\nabla}{\rightarrow} X$ is an isomorphism for all $X \in \mathcal{C}$.

The following is the prototypical example of acyclicity:

Example 2.4.5 (Bousfield). For $E \in \mathrm{Sp}$, we can consider the $\infty$-category $\mathrm{Sp}_{E}$ of $E$-local spectra. By Lemma 2.4.4, a space is $\mathrm{Sp}_{E}$-acyclic if and only if it is $E$-acyclic in the sense of Bousfield, i.e. has the $E$-homology of a point.

Remark 2.4.6. For an $\infty$-category $\mathcal{C}$, a space $A$ is $\mathcal{C}$-acyclic if the following equivalent conditions hold:

(1) The fold map $X[A] \stackrel{\nabla}{\rightarrow} X$ is an isomorphism for all $X \in \mathcal{C}$.

(2) The diagonal map $X \stackrel{\Delta}{\rightarrow} X^{A}$ is an isomorphism for all $X \in \mathcal{C}$.

We warn the reader that for an individual object $X$, it can happen that the map $X \stackrel{\Delta}{\rightarrow} X^{A}$ is an isomorphism, but $X[A] \stackrel{\nabla}{\rightarrow} X$ is not (and vise versa). As a trivial example, consider $\mathcal{C}=\mathcal{S}$ with $X=\mathrm{pt}$ and any $A \neq \mathrm{pt}$. 


\section{Relation to amenability}

Under suitable ambidexterity assumptions, the notion of $\mathcal{C}$-acyclicity turns out to be closely related to that of $\mathcal{C}$-amenability. To begin with, recall that for a $\mathcal{C}$-ambidextrous space $A$ and an object $X \in \mathcal{C}$, the map $|A|_{X}$ is given by the composition

$$
X \stackrel{\Delta}{\rightarrow} X^{A} \stackrel{\mathrm{Nm}_{A}^{-1}}{\longrightarrow} X[A] \stackrel{\nabla}{\longrightarrow} X .
$$

Thus, if $A$ is $\mathcal{C}$-acyclic, then it is in particular $\mathcal{C}$-amenable. However, there is a deeper connection between acyclicity and amenability, which we first state on an object-wise level:

Proposition 2.4.7. Let $\mathcal{C} \in \mathrm{Cat}_{\infty}$ and let $A$ be a connected $\mathcal{C}$-ambidextrous space. For every $X \in \mathcal{C}$, the following are equivalent:

(1) The fold map $X[A] \stackrel{\nabla}{\rightarrow} X$ is an isomorphism.

(2) The diagonal $X \stackrel{\Delta}{\rightarrow} X^{A}$ is an isomorphism.

(3) $|\Omega A|_{X}$ is invertible.

Moreover, $|A|_{X}$ is then the inverse of $|\Omega A|_{X}$.

Proof. Let pt $\stackrel{e}{\rightarrow} A$ be a base point. First, we show that if the diagonal map $X \stackrel{\Delta}{\rightarrow} X^{A}$ is an isomorphism, then $|A|_{X}|\Omega A|_{X}=\operatorname{Id}_{X}$. We begin by reducing the claim to the fact that the map

$$
|e|_{A^{*} X}: A^{*} X \rightarrow A^{*} X
$$

equals the constant map on $|\Omega A|_{X}$. Indeed, given that, by integrating along $A$, we get ([CSY18, Propositions 2.1.15 and 3.1.13])

$$
\operatorname{Id}_{X}=\int_{A \circ e} \operatorname{Id}_{e^{*} A^{*} X}=\int_{A}\left(\int_{e} \operatorname{Id}_{e^{*} A^{*} X}\right)=\int_{A}|e|_{A^{*} X}=\int_{A} A^{*}\left(|\Omega A|_{X}\right)=|A|_{X}|\Omega A|_{X} .
$$

Now, recall that the diagonal $X \stackrel{\Delta}{\rightarrow} X^{A}=A_{*} A^{*} X$ is the unit $u_{*}^{A}$ of the adjunction $A^{*}: \mathcal{C} \leftrightarrows \mathcal{C}^{A}: A_{*}$. Thus, if $u_{*}^{A}$ is an isomorphism at $X$, then the map

$$
\operatorname{Map}(X, X) \rightarrow \operatorname{Map}\left(A^{*} X, A^{*} X\right)
$$

is an isomorphism. Since $e^{*} A^{*}=\mathrm{Id}$, it follows by 2-out-of-3 that the map

$$
\operatorname{Map}\left(A^{*} X, A^{*} X\right) \rightarrow \operatorname{Map}\left(e^{*} A^{*} X, e^{*} A^{*} X\right)=\operatorname{Map}(X, X)
$$

is an isomorphism as well. Thus, it suffices to check that the maps $|e|_{A^{*} X}$ and $A^{*}\left(|\Omega A|_{X}\right)$ coincide after applying $e^{*}$. The pullback square of spaces

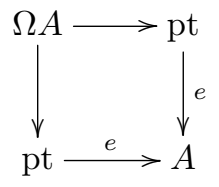


gives by [CSY18, Proposition 3.1.13]

$$
e^{*}\left(|e|_{A^{*} X}\right)=|\Omega A|_{e^{*} A^{*} X}=|\Omega A|_{X} .
$$

This concludes the proof of $(2) \Longrightarrow(3)$ and that $|A|_{X}$ is the inverse of $|\Omega A|_{X}$. A completely symmetric argument using the adjunction $A_{!} \dashv A^{*}$ instead of $A^{*} \dashv A_{*}$ shows that $(1) \Longrightarrow(3)$ and that $|A|_{X}$ is the inverse of $|\Omega A|_{X}$. The implication (3) $\Longrightarrow(1)$ in the case that $|\Omega A|_{X}$ is invertible for all $X \in \mathcal{C}$ is given by [CSY18, Proposition 3.1.18]. One easily checks that all the arguments are, in fact, object-wise. Alternatively, one can run the argument on the full subcategory of objects on which $|\Omega A|$ is invertible. In particular, each of the conditions (1), (2), and (3) implies that $|A|_{X}$ is the inverse of $|\Omega A|_{X}$ and so, in particular, is invertible. Finally, the composition

$$
X \stackrel{\Delta}{\rightarrow} X^{A} \stackrel{\mathrm{Nm}_{A}}{\longrightarrow} X[A] \stackrel{\nabla}{\rightarrow} X
$$

is $|A|_{X}$. Thus, by 2-out-of-3, we also have the implication $(1) \Longrightarrow(2)$.

From this we get:

Corollary 2.4.8. Let $\mathcal{C} \in \mathrm{Cat}_{\infty}$ and let $A$ be a connected space. The following conditions are equivalent:

(1) A is $\mathcal{C}$-acyclic and $\mathcal{C}$-ambidextrous.

(2) $\Omega A$ is $\mathcal{C}$-amenable and $\mathcal{C}$ admits $A$-colimits.

In which case $|A|=|\Omega A|^{-1}$.

Proof. This follows from Proposition 2.4.7 and the fact that if $\Omega A$ is $\mathcal{C}$-amenable, then $A$ is $\mathcal{C}$ ambidextrous by [CSY18, Proposition 3.1.17].

We note that the assumption of $\mathcal{C}$-ambidexterity in (1) of Corollary 2.4 .8 can not be relaxed to weak $\mathcal{C}$-ambidexterity. The following is a simple counter-example:

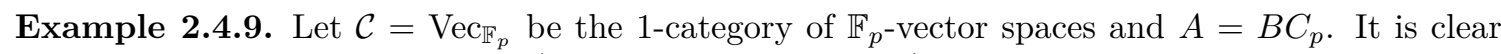
that $A$ is weakly $\mathcal{C}$-ambidextrous (as $\operatorname{Vec}_{\mathbb{F}_{p}}$ is semiadditive) and that $B C_{p}$ is $\mathcal{C}$-acyclic. However, $B C_{p}$ is not $\mathcal{C}$-ambidextrous (and $C_{p}$ is not $\mathcal{C}$-amenable).

Remark 2.4.10. The notion of acyclicity can be considered for a general (not necessarily $\pi$-finite) space in any $\infty$-category, without any assumptions on ambidexterity. However, in the presence of ambidexterity, Corollary 2.4.8 allows us to deduce the acyclicity of a given $\pi$-finite space from the amenability of its loop space. This strategy was already employed in the proof of [CSY18, Theorem E] (exploiting the $\infty$-semiadditivity of $\left.\mathrm{Sp}_{T(n)}\right)$.

We conclude this subsection by showing an analogue of the equivalence of (1) and (3) in Proposition 2.4.7, for the notions of $\mathcal{C}$-acyclicity and $\mathcal{C}$-triviality. As before, it is clear that a $\mathcal{C}$-trivial space is, in particular, $\mathcal{C}$-acyclic, but there is a better statement:

Proposition 2.4.11. Let $A$ be a connected space and let $\mathcal{C}$ be an $\infty$-category which admits $\Omega A$ colimits. Then, $A$ is $\mathcal{C}$-trivial if and only if $\Omega A$ is $\mathcal{C}$-acyclic. 
Proof. Let $\mathrm{pt} \stackrel{e}{\rightarrow} A$ be a base point. The composition

$$
\mathcal{C} \stackrel{A^{*}}{\longrightarrow} \mathcal{C}^{A} \stackrel{e^{*}}{\longrightarrow} \mathcal{C}
$$

is the identity. This implies that $A^{*}$ is an equivalence if and only if $e^{*}$ is. Moreover, it also implies that $e^{*}$ is essentially surjective and hence an equivalence if and only if it is fully faithful. Namely, if and only if $e$ is $\mathcal{C}$-acyclic. Since $\mathcal{C}$-acyclicity is a fiber-wise condition (Proposition 2.4.2), $e$ is $\mathcal{C}$-acyclic if and only if $\Omega A$ is $\mathcal{C}$-acyclic.

\section{Height}

In this section, we introduce the notion of "semiadditive height" for objects in higher semiadditive $\infty$-categories, which is the central object of study in this paper. We establish here the most general properties of this notion, while those related to stability will be differed to the next section.

\subsection{Semiadditive Height}

The definition of height for a higher semiadditive $\infty$-category depends on a choice of a prime $p$. In fact, it suffices to have a certain " $p$-typical" version of $m$-semiadditivity, in which one requires ambidexterity only for $m$-finite $p$-spaces. To emphasize the relevant structure, and for some future applications, we shall develop the basic theory of height in this level of generality. However, for an $\infty$-category $\mathcal{C}$ which is 0 -semiadditive and $p$-local, the " $p$-typical" version of higher semiadditivity will turn out to be equivalent to ordinary higher semiadditivity (Proposition 3.2.6). Thus, for the applications considered in this paper, this point is of minor importance.

\section{p-Typical semiadditivity}

We begin with a definition of the following " $p$-typical" version of higher semiadditivity:

Definition 3.1.1. Let $p$ be a prime and $0 \leq m \leq \infty$. We say that

(1) An $\infty$-category $\mathcal{C}$ is $p$-typically $m$-semiadditive if all $m$-finite $p$-spaces are $\mathcal{C}$-ambidextrous.

(2) A functor $F: \mathcal{C} \rightarrow \mathcal{D}$ between such is $p$-typically $m$-semiadditive if it preserves all $m$-finite $p$-space colimits.

(3) An $\mathcal{O}$-monoidal $\infty$-category $\mathcal{C}$, for some $\infty$-operad $\mathcal{O}$, is $p$-typically $m$-semiadditively $\mathcal{O}$ monoidal if it is $p$-typically $m$-semiadditive and is compatible with $m$-finite $p$-space colimits.

We denote by $\mathrm{Cat}^{\oplus{ }^{\oplus-m}} \subset$ Cat $_{\infty}$ the subcategory of $p$-typically $m$-semiadditive $\infty$-categories and $p$-typically $m$-semiadditive functors.

It is clear that an $m$-semiadditive $\infty$-category or functor are also $p$-typically $m$-semiadditive for every prime $p$. It is useful to know that to verify $p$-typical $m$-semiadditivity, it suffices to consider only the "building blocks":

Proposition 3.1.2. Let $0 \leq m \leq \infty$. 
(1) An $\infty$-category $\mathcal{C} \in \mathrm{Cat}^{\oplus-0}$ is p-typically $m$-semiadditive if and only if $B^{k} C_{p}$ is $\mathcal{C}$-ambidextrous for all $k=1, \ldots, m$.

(2) For $\mathcal{C}, \mathcal{D} \in \mathrm{Cat}_{\infty}^{\oplus_{p}-m}$, a 0-semiadditive functor $F: \mathcal{C} \rightarrow \mathcal{D}$ is $p$-typically $m$-semiadditive if and only if it preserves $B^{k} C_{p^{-}}$(co) limits for all $k=1, \ldots, m$.

Proof. (1) The "only if" part is clear. Conversely, we need to show that if $B^{k} C_{p}$ is $\mathcal{C}$-ambidextrous for all $k=1, \ldots, m$, then every $m$-finite $p$-space $A$ is $\mathcal{C}$-ambidextrous. Since $\mathcal{C}$ is 0 -semiadditive, we are reduced to the case that $A$ is connected. The Postnikov tower of $A$ can be refined to a tower of principal fibrations

$$
A=A_{r} \rightarrow \cdots \rightarrow A_{1} \rightarrow A_{0}=\mathrm{pt},
$$

such that the fiber of each $A_{i} \rightarrow A_{i-1}$ is of the form $B^{k_{i}} C_{p}$ for some $1 \leq k_{i} \leq m$. To show that $A$ is $\mathcal{C}$-ambidextrous, it suffices to show that each $A_{i} \rightarrow A_{i-1}$ is $\mathcal{C}$-ambidextrous (as $\mathcal{C}$-ambidextrous maps are closed under composition). Finally, since $\mathcal{C}$-ambidexterity is a fiber-wise condition, this follows from the fact that $B^{k_{i}} C_{p}$ is $\mathcal{C}$-ambidextrous.

(2) Follows by an analogous argument to (1).

In a $p$-typically $m$-semiadditive $\infty$-category $\mathcal{C}$, one can discuss cardinalities of $m$-finite $p$-spaces. As one might expect, the Eilenberg-MacLane spaces $B^{n} C_{p}$ play a fundamental role, and so deserve a special notation:

Definition 3.1.3. Let $\mathcal{C} \in \mathrm{Cat}^{\oplus^{-}-m}$. For every integer $0 \leq n \leq m$, we define $p_{(n)}^{\mathcal{C}}:=\left|B^{n} C_{p}\right|$ as a natural endomorphism of the identity functor of $\mathcal{C}$. We shall omit the superscript " $\mathcal{C}$ ", whenever $\mathcal{C}$ is clear from the context.

A fundamental example to keep in mind is the following:

Example 3.1.4. For $\Theta_{n}=\operatorname{Mod}_{E_{n}}\left(\operatorname{Sp}_{K(n)}\right)$, we have by Proposition 2.2.5:

$$
p_{(k)}^{\Theta_{n}}:=\left|B^{k} C_{p}\right|_{n}=p^{\left(\begin{array}{c}
n-1 \\
k
\end{array}\right)}
$$

for all $n, k \geq 0$.

\section{Semiadditive height}

In what follows it will be convenient to use the following terminology:

Definition 3.1.5. Let $\mathcal{C} \in \mathrm{Cat}_{\infty}$ and let $\alpha: \operatorname{Id}_{\mathcal{C}} \rightarrow \operatorname{Id}_{\mathcal{C}}$ be a natural endomorphism. An object $X \in \mathcal{C}$ is called

(1) $\alpha$-divisible if $\alpha_{X}$ is invertible.

(2) $\alpha$-complete if $\operatorname{Map}(Z, X) \simeq$ pt for all $\alpha$-divisible $Z$.

We denote by $\mathcal{C}\left[\alpha^{-1}\right]$ and $\widehat{\mathcal{C}}_{\alpha}$ the full subcategories of $\mathcal{C}$ spanned by the $\alpha$-divisible and $\alpha$-complete objects respectively.

Using the operations $p_{(n)}$ we can now define the semiadditive height: 
Definition 3.1.6. Let $\mathcal{C} \in \mathrm{Cat}^{\oplus^{p}-m}$ and let $0 \leq n \leq m<\infty$. For every $X \in \mathcal{C}$ we define and denote the (semiadditive) height of $X$ as follows:

(1) $\operatorname{ht}_{\mathcal{C}}(X) \leq n$, if $X$ is $p_{(n)}^{\mathcal{C}}$-divisible.

(2) $\mathrm{ht}_{\mathcal{C}}(X)>n$, if $X$ is $p_{(n)}^{\mathcal{C}}$-complete.

(3) $\operatorname{ht}_{\mathcal{C}}(X)=n$, if ht ${ }_{\mathcal{C}}(X) \leq n$ and ht ${ }_{\mathcal{C}}(X)>n-1$.

We also extend the definition to $n=m=\infty$ as follows. For every $X \in \mathcal{C}$, we write ht ${ }_{\mathcal{C}}(X)=\infty$ if and only if $h_{\mathcal{C}}(X)>k$ for all $k \in \mathbb{N}$. Additionally, by convention $-1<\mathrm{ht}_{\mathcal{C}}(X) \leq \infty$ for all $X$, and $\operatorname{ht}_{\mathcal{C}}(X) \leq-1$ or ht ${ }_{\mathcal{C}}(X)>\infty$ if and only if $X=0$. We shall drop the subscript $\mathcal{C}$ in ht ${ }_{\mathcal{C}}$, when the $\infty$-category is clear from the context.

Remark 3.1.7. We emphasize that the notation $\mathrm{ht}(X) \leq n$ (and similarly ht $(X)>n$ etc.) asserts that $X$ satisfies a certain property, and does not mean that $h t(X)$ is a well-defined number, which can be compared with $n$. We note that the only object in $\mathcal{C}$, which can simultaneously have height $\leq n$ and $>n$ is the zero object.

The motivating example for the definition of height is the following:

Example 3.1.8. Let $\mathcal{C}$ be a 0 -semiadditive $\infty$-category. An object $X \in \mathcal{C}$ is of height 0 if and only if $p=p_{(0)}$ acts invertibly on $X$, and of height $>0$ if it is $p$-complete.

The first thing to show is that the notion of height behaves as the terminology suggests:

Proposition 3.1.9. Let $\mathcal{C} \in \mathrm{Cat}^{\oplus_{p}-m}$ and let $0 \leq n_{0} \leq n_{1} \leq m$ be some integers. For every $X \in \mathcal{C}$,

(1) If ht $(X) \leq n_{0}$, then $\operatorname{ht}(X) \leq n_{1}$.

(2) If ht $(X)>n_{1}$, then $\operatorname{ht}(X)>n_{0}$.

Proof. To prove (1), it suffices to show that if $\operatorname{ht}(X) \leq n$ for some $n \leq m-1$, then $\operatorname{ht}(X) \leq n+1$. We consider the principal fiber sequence

$$
B^{n} C_{p} \rightarrow \mathrm{pt} \rightarrow B^{n+1} C_{p} .
$$

All maps and spaces in this sequence are $\mathcal{C}$-ambidextrous by assumption. Since $\operatorname{ht}(X) \leq n$, we have that $\left|B^{n} C_{p}\right|_{X}$ is invertible. By Proposition 2.3.3, we get

$$
\left|B^{n+1} C_{p}\right|_{X}\left|B^{n} C_{p}\right|_{X}=|\mathrm{pt}|_{X}=\operatorname{Id}_{X} .
$$

Thus, $\left|B^{n+1} C_{p}\right|_{X}$ is invertible as well, and hence ht $(X) \leq n+1$. Claim (2) now follows from (1) by definition.

Remark 3.1.10. In any stable $\infty$-category $\mathcal{C}$, a non-zero object can have height $>0$ for at most one prime $p$. In particular, if $\mathcal{C}$ is $p$-local, every object has height 0 for every prime $\ell \neq p$ (in fact, this is 'if an only if'). Nevertheless, in this case the notion of $\ell$-height with respect to different primes $\ell$ allows one to treat "prime to $p$ phenomena" as "height 0 phenomena" for primes $\ell \neq p$. 
It is also useful to consider the corresponding subcategories of objects having height in a certain range:

Definition 3.1.11. Let $\mathcal{C} \in \mathrm{Cat}^{\oplus p-m}$ and let $0 \leq n \leq m \leq \infty$. We define

$$
\begin{gathered}
\mathcal{C}_{\leq n}=\mathcal{C}\left[p_{(n)}^{-1}\right] \quad, \quad \mathcal{C}_{>n}=\widehat{\mathcal{C}}_{p_{(n)}}, \\
\left.\mathcal{C}_{n}=\mathcal{C}_{\leq n} \cap \mathcal{C}_{>n-1}=\widehat{\mathcal{C}\left[p_{(n)}^{-1}\right]}\right]_{p_{(n-1)}}=\widehat{\mathcal{C}}_{p_{(n-1)}}\left[p_{(n)}^{-1}\right] .
\end{gathered}
$$

the full subcategories of $\mathcal{C}$ spanned by objects of height $\leq n,>n$ and $n$ respectively. We also write $\operatorname{Ht}(\mathcal{C}) \leq n,>n$ or $n$, if $\mathcal{C}=\mathcal{C}_{\leq n}, \mathcal{C}_{>n}$ or $\mathcal{C}_{n}$ respectively.

The above defined subcategories are themselves $p$-typically $m$-semiadditive:

Proposition 3.1.12. Let $\mathcal{C} \in$ Cat $^{\oplus_{p}-m}$. For every $n=0, \ldots, m$, the full subcategories $\mathcal{C}_{\leq n}, \mathcal{C}_{>n}$ and $\mathcal{C}_{n}$ are closed under limits in $\mathcal{C}$. In particular, they are p-typically $m$-semiadditive, and are furthermore $m$-semiadditive if $\mathcal{C}$ is.

Proof. An object $X \in \mathcal{C}$ belongs to $\mathcal{C}_{\leq n}$ if and only if $X$ is $p_{(n)}$-divisible. Thus $\mathcal{C}_{\leq n}$ is closed under all limits which exist in $\mathcal{C}$. By definition, $\mathcal{C}_{>n}$ and $\mathcal{C}_{\infty}$ are closed under limits in $\mathcal{C}$ as well. Thus, for $n<\infty$ we have that $\mathcal{C}_{n}=\mathcal{C}_{\leq n} \cap \mathcal{C}_{>n-1}$ is also closed under limits in $\mathcal{C}$. Finally, by Proposition 2.1.4(3), it follows that all these subcategories are $p$-typically $m$-semiadditive and are furthermore $m$-semiadditive if $\mathcal{C}$ is.

Next, we consider the behavior of height with respect to higher semiadditive functors. It turns out that the height can only go down:

Proposition 3.1.13. Let $F: \mathcal{C} \rightarrow \mathcal{D}$ be a map in Cat $^{\oplus_{p}-m}$. For all $X \in \mathcal{C}$ and $0 \leq n \leq m$, if $\operatorname{ht}_{\mathcal{C}}(X) \leq n$ then $\operatorname{ht}_{\mathcal{D}}(F(X)) \leq n$. If $F$ is conservative, then the converse holds as well.

Proof. This follows immediately from the fact that $F$ maps $p_{(n)}^{\mathcal{C}}$ to $p_{(n)}^{\mathcal{D}}$.

In contrast, the following example shows that a higher semiadditive functor need not preserve lower bounds on height:

Example 3.1.14. The 0 -semiadditive functor $L_{\mathbb{Q}}: \mathrm{Sp}_{(p)} \rightarrow \mathrm{Sp}_{\mathbb{Q}}$ maps the $p$-complete sphere $\widehat{\mathbb{S}}_{p}$, which is of height $>0$, to a non-zero object $\mathbb{Q} \otimes \widehat{\mathbb{S}}_{p}$ of height 0 .

For an inclusion of a full subcategory, we can say a bit more:

Proposition 3.1.15. Let $\mathcal{C} \in \mathrm{Cat}^{\oplus_{p}-m}$ and let $\mathcal{C}^{\prime} \subseteq \mathcal{C}$ be a full subcategory closed under $m$-finite p-space (co)limits. Given $X \in \mathcal{C}^{\prime}$ and $0 \leq n \leq m$, we have

(1) $\mathrm{ht}_{\mathcal{C}^{\prime}}(X) \leq n$ if and only if $\mathrm{ht}_{\mathcal{C}}(X) \leq n$.

(2) $\operatorname{ht}_{\mathcal{C}}(X)>n$ implies ht $\mathcal{C}^{\prime}(X)>n$.

Proof. (1) follows from Proposition 3.1.13 applied to the inclusion $\mathcal{C}^{\prime} \hookrightarrow \mathcal{C}$. For (2), if $h_{\mathcal{C}}(X)>$ $n$, then for every $Z \in \mathcal{C}_{\leq n}$, we have $\operatorname{Map}(Z, X) \simeq$ pt. We now observe that by (1), we have $\mathcal{C}_{\leq n}^{\prime}=\mathcal{C}^{\prime} \cap \mathcal{C}_{\leq n}$. Thus, for every $Z^{\prime} \in \mathcal{C}_{\leq n}^{\prime}$, we have $\operatorname{Map}\left(Z^{\prime}, X\right) \simeq$ pt, which by definition means $\mathrm{ht}_{\mathcal{C}^{\prime}}(X)>n$. 
In presence of a $p$-typically $m$-semiadditively monoidal structure, an upper bound on the height of the unit implies an upper bound on the height of the $\infty$-category:

Corollary 3.1.16. Let $\mathcal{C}$ be $p$-typically m-semiadditively monoidal $\infty$-category. For every $0 \leq n \leq$ $m$, we have $\mathrm{Ht}(\mathcal{C}) \leq n$ if and only if $\mathrm{ht}_{\mathcal{C}}(\mathbb{1}) \leq n$.

Proof. Given $X \in \mathcal{C}$, the functor $X \otimes(-): \mathcal{C} \rightarrow \mathcal{C}$ is $p$-typically $m$-semiadditive. Thus, the claim follows from Proposition 3.1.13.

\subsection{Bounded Height}

In this subsection, we study the implications for a higher semiadditive $\infty$-category of having bounded height. These results generalize the previously discussed facts regarding the $\infty$-semiadditive structure of semirational $\infty$-categories (i.e. of height 0 ), and fall under the slogan that "the higher semiadditive structure is trivial above the height". As a by-product, we shall see that for a 0semiadditive $p$-local $\infty$-category, there is no difference between $m$-semiadditivity and $p$-typical $m$-semiadditivity.

\section{Amenability \& acyclicity}

The results on amenability and acyclicity from section 2.4 imply the following equivalent characterizations of height $\leq n$ :

Proposition 3.2.1. Let $\mathcal{C} \in \mathrm{Cat}^{\oplus_{p}-n}$, which admits $B^{n+1} C_{p^{-}}($co)limits. The following properties are equivalent:

(1) $\operatorname{Ht}(\mathcal{C}) \leq n$ (i.e. $B^{n} C_{p}$ is $\mathcal{C}$-amenable).

(2) $B^{n+1} C_{p}$ is $\mathcal{C}$-acyclic.

(3) $B^{n+2} C_{p}$ is $\mathcal{C}$-trivial.

We can therefore characterize the height of an $\infty$-category in ways that do not make an explicit reference to the higher semiadditive structure.

Proof. The equivalence of (1) and (2) follows from Corollary 2.4.8 and the equivalence of (2) and (3) from Proposition 2.4.11.

The following can be seen as a ( $p$-typical) generalization of the fact that a semirational $\infty$-category is automatically $\infty$-semiadditive:

Proposition 3.2.2. Let $\mathcal{C} \in \mathrm{Cat}^{\oplus p-n}$ such that $\mathrm{Ht}(\mathcal{C}) \leq n$, and assume $\mathcal{C}$ admits $B^{n+1} C_{p}$-(co)limits. Then, $\mathcal{C}$ is p-typically $\infty$-semiadditive.

Proof. By Proposition 3.1.2(1), it suffice to prove that

(*) $B^{k} C_{p}$ is $\mathcal{C}$-amenable (and ,in particular, $\mathcal{C}$-ambidextrous) and $\mathcal{C}$ admits all $B^{k+1} C_{p}$-colimits. 
holds for all $k \geq n$. We shall prove this by induction on $k$. The base case $k=n$ is given by assumption. Assume $(*)$ holds for some $k \geq n$ and consider the fiber sequence

$$
B^{k} C_{p} \rightarrow \mathrm{pt} \rightarrow B^{k+1} C_{p} .
$$

By the inductive hypothesis, $\left|B^{k} C_{p}\right|$ is invertible and $\mathcal{C}$ admits $B^{k+1} C_{p^{-}}(\mathrm{co})$ limits. Therefore by [CSY18, Propostion 3.1.17], the space $B^{k+1} C_{p}$ is $\mathcal{C}$-ambidextrous. Moreover, by Proposition 2.3.3, $\left|B^{k+1} C_{p}\right|$ is invertible as well. Finally, by Proposition 3.2.1(3), the diagonal functor $\mathcal{C} \rightarrow \mathcal{C}^{B^{k+2} C_{p}}$ is an equivalence and hence in particular $\mathcal{C}$ admits $B^{k+2} C_{p^{-}}$(co)limits.

We now show that claims (1)-(3) of Proposition 3.2.1 extend to much wider classes of spaces:

Proposition 3.2.3. Let $\mathcal{C} \in \mathrm{Cat}_{\infty}^{\oplus_{p}-\infty}$ such that $\mathrm{Ht}(\mathcal{C}) \leq n$. For every $\pi$-finite p-space $A$, the following hold:

(1) If $A$ is $(n-1)$-connected, then $A$ is $\mathcal{C}$-amenable.

(2) If $A$ is n-connected, then $A$ is $\mathcal{C}$-acyclic.

(3) If $A$ is $(n+1)$-connected, then $A$ is $\mathcal{C}$-trivial.

Proof. For (1), let $A$ be an $(n-1)$-connected $\pi$-finite $p$-space. The Postnikov tower of $A$ can be refined to a tower of principal fibrations

$$
A=A_{r} \rightarrow \cdots \rightarrow A_{1} \rightarrow A_{0}=\mathrm{pt},
$$

such that the fiber of each $A_{i} \rightarrow A_{i-1}$ is of the form $B^{k_{i}} C_{p}$ for some $k_{i} \geq n$. Since we assumed $\operatorname{Ht}(\mathcal{C}) \leq n$, we also have $\operatorname{Ht}(\mathcal{C}) \leq k_{i}$ (Proposition 3.1.9), and hence all the spaces $B^{k_{i}} C_{p}$ are $\mathcal{C}$ amenable. By Corollary 2.3.5, the class of $\mathcal{C}$-amenable spaces is closed under principal extensions, and therefore $A$ is $\mathcal{C}$-amenable. Now, (2) follows from Corollary 2.4 .8 and (1) applied to $\Omega A$. Similarly, (3) follows from Proposition 2.4.11 and (2) applied to $\Omega A$.

Example 3.2.4. Let $\mathcal{C}$ be semirational, and so in particular of height 0 ( $\mathrm{such}$ as $\mathrm{Vec}_{\mathbb{Q}}$ or $\mathrm{Sp}_{\mathbb{Q}}$ ). By Example 2.2.2, for every $\pi$-finite $p$-space $A$, the cardinality $|A|$ is a sum of positive rational numbers. Thus, $|A|$ is invertible if and only if $A$ is non-empty (i.e. (-1)-connected). The map $X \rightarrow X^{A}$ is an equivalence for all $X$, if and only if $A$ is connected, and $\mathcal{C} \rightarrow \mathcal{C}^{A}$ is an equivalence if and only if $A$ is simply-connected.

\section{Cardinality}

Recall from Example 2.2.2, that the formula for the homotopy cardinality (1) could be deduced solely from the "multiplicativity property" with respect to fiber sequences. For higher heights, we have the following analogue:

Proposition 3.2.5. Let $\mathcal{C} \in \mathrm{Cat}^{\oplus^{p}-\infty}$ such that $\operatorname{Ht}(\mathcal{C}) \leq n$. Given a principal fibration of $\pi$-finite p-spaces

$$
F \rightarrow A \rightarrow B
$$

if $F$ is $(n-1)$-connected, then $|A|=|F| \cdot|B|$. In particular,

$$
p_{(k)}=p_{(n)}^{(-1)^{k-n}}, \quad \forall k \geq n .
$$


Proof. By Proposition 3.2.3(1), the space $F$ is $\mathcal{C}$-amenable. Thus, by Proposition 2.3.3, we have $|A|=|F| \cdot|B|$. By an inductive application of this to the fiber sequence

$$
B^{k} C_{p} \rightarrow \mathrm{pt} \rightarrow B^{k+1} C_{p},
$$

we obtain the formula $p_{(k)}=p_{(n)}^{(-1)^{k-n}}$ for all $k \geq n$.

Furthermore, using Proposition 3.2.5 together with a principal refinement of the Postnikov tower (as in the proof of Proposition 3.2.2), one can reduce the computation of the $\mathcal{C}$-cardinality of all $\pi$-finite $p$-spaces to those of connected $n$-finite $p$-spaces.

\section{The $p$-local Case}

In many situations of interest, the $\infty$-category $\mathcal{C}$ under consideration is 0 -semiadditive, $p$-local and admits all 1 -finite colimits. In this case, $\mathcal{C}$ is automatically $\ell$-typically $\infty$-semiadditive for all primes $\ell \neq p$ (Proposition 3.2.2). Moreover, in this case there is essentially no difference between higher semiadditivity and $p$-typical higher semiadditivity:

Proposition 3.2.6. Let $\mathcal{C}$ be a 0 -semiadditive $p$-local $\infty$-category which admits all 1-finite limits and colimits. The $\infty$-category $\mathcal{C}$ is p-typically $m$-semiadditive if and only if it is $m$-semiadditive.

Proof. Assume that $\mathcal{C}$ is $p$-typically $m$-semiadditive. By [HL13, Proposition 4.4.16] and the fact that the space $B C_{p}$ is $\mathcal{C}$-ambidextrous, we get that $\mathcal{C}$ is 1-semiadditive. To get higher semiadditivity, we first have by assumption that $\mathcal{C}$ is $p$-typically $m$-semiadditive. Moreover, since $\mathcal{C}$ is $p$-local, it is $\ell$-typically $m$-semiadditive for all $\ell \neq p$ (Proposition 3.2.2). Thus, $B^{k} C_{\ell}$ is $\mathcal{C}$-ambidextrous for all primes $\ell$ and all integers $k=2, \ldots m$. By inductive application of [HL13, Proposition 4.4.19], it follows that $\mathcal{C}$ is $m$-semiadditive. We note that in both [HL13, Proposition 4.4.16] and [HL13, Proposition 4.4.19], one assumes that $\mathcal{C}$ admits all small limits and colimits. However, the proofs use only the limits and colimits which we assumed in the statement.

When applied to $p$-local $\infty$-categories, the main results of this subsection can be summarized as follows:

Theorem 3.2.7. Let $\mathcal{C}$ be a 0 -semiadditive $p$-local $\infty$-category, which admits all $(n+1)$-finite limits and colimits. If $\mathcal{C}$ is p-typically $n$-semiadditive such that $\operatorname{Ht}(\mathcal{C}) \leq n$, then $\mathcal{C}$ is $\infty$-semiadditive. Moreover, for every $\pi$-finite space A:

(1) If $A$ is $(n-1)$-connected and nilpotent, then $A$ is $\mathcal{C}$-amenable.

(2) If $A$ is n-connected, then $A$ is $\mathcal{C}$-acyclic.

(3) If $A$ is $(n+1)$-connected, then $A$ is $\mathcal{C}$-trivial.

Proof. Since $\operatorname{Ht}(\mathcal{C}) \leq n$ and $\mathcal{C}$ admits $B^{n+1} C_{p^{-}}$limits and colimits, it follows that $\mathcal{C}$ is $p$-typically $\infty$-semiadditive (Proposition 3.2.2). Since $\mathcal{C}$ is $p$-local, it follows that $\mathcal{C}$ is in fact $\infty$-semiadditive (Proposition 3.2.6). For (1), we observe that if $A$ is $\pi$-finite and nilpotent, then $A=\prod_{\ell} A_{(\ell)}$ where $\ell$ ranges over primes and $A_{(\ell)}$ is a $\pi$-finite $\ell$-space which is contractible for almost all $\ell$ (e.g. [PS17, Theorem 5.7]). Since we have $|A|=\prod_{\ell}\left|A_{(\ell)}\right|$ (Remark 2.1.10), the $\mathcal{C}$-amenability of $A$ 
follows from the $\mathcal{C}$-amenability of all the $A_{(\ell)}$-s (see Proposition 3.2.3(1)). Finally, as in the proof of Proposition 3.2.3, (2) follows from (1) and Corollary 2.4.8 applied to $\Omega A$ and (3) follows from (2) and Proposition 2.4.11.

Remark 3.2.8. We note that the nilpotence condition in Theorem 3.2.7(1) is vacuous for simply connected spaces and hence relevant only for height $n=1$. However, in this case it can not be dropped. Indeed, by Example 2.3.7, at the prime $p=2$ we have $\left|B \Sigma_{3}\right|_{1}=\frac{2}{3}$, which is not-invertible. With a little more effort one can show that if $\operatorname{Ht}(\mathcal{C}) \leq 1$, then $|A|$ is invertible for every connected $\pi$ finite space $A$, such that that the $p$-Sylow subgroup of $\pi_{1} A$ is normal. In other words, the $p$-primary fusion in the fundamental group is the only obstruction for the invertibility of $|A|$.

\subsection{Semiadditive Redshift}

By Example 2.2.8, the $\infty$-category $\mathrm{Cat}_{m \text {-fin }}$ is $m$-semiadditive and hence given $\mathcal{C} \in \mathrm{Cat}_{m \text {-fin }}$, we can discuss $\operatorname{ht}(\mathcal{C})$ for various primes $p$, which is the height of $\mathcal{C}$ as an object of $\mathrm{Cat}_{m \text {-fin }}$. However, if $\mathcal{C}$ itself is $p$-typically higher semiadditive, then we have also defined $\mathrm{Ht}(\mathcal{C})$, as the height of the objects of $\mathcal{C}$. These two notions of height do not coincide. Rather, we shall now show that $h t(\mathcal{C})$ exceeds $\mathrm{Ht}(\mathcal{C})$ exactly by one. As the semiadditive height generalizes the chromatic height, this can be viewed as a particular manifestation of the "redshift" principle. Roughly speaking, categorification tends to shift the height up by one. Before we begin, we need a general categorical lemma:

Lemma 3.3.1. Let $F: \mathcal{C} \leftrightarrows \mathcal{D}: G$ be an adjunction. If $G F$ is an equivalence, then $F$ is fully faithful.

Proof. The functor $F$ is fully faithful if and only if the unit Id $\stackrel{u}{\rightarrow} G F$ is an isomorphism. The unit is part of a monad structure on $G F$ making it a monoid in the homotopy category of $\operatorname{Fun}(\mathcal{C}, \mathcal{C})$, whose monoidal structure is given by composition. It therefore suffices to show that given a monoid $M$ in any monoidal (ordinary) category, if $M$ is invertible with respect to the monoidal structure, then the unit map $\mathbb{1} \stackrel{u}{\rightarrow} M$ is an isomorphism. We observe that the multiplication map $M \otimes M \stackrel{m}{\rightarrow} M$ is always a left inverse of $1 \otimes u$. Thus, since $M \otimes(-)$ is an equivalence, it follows that $u$ admits a left inverse as well. To conclude the proof, it remains to show that $u$ admits a right inverse. Since $M$ is invertible, it is in particular dualizable, and the dual $M^{\vee}$ of $M$ is its inverse. More precisely, the duality datum maps $\mathbb{1} \stackrel{\eta}{\rightarrow} M \otimes M^{\vee}$ and $M^{\vee} \otimes M \stackrel{\varepsilon}{\rightarrow} \mathbb{1}$ are isomorphisms and exhibit $M^{\vee}$ as the inverse of $M$. Consider the following commutative diagram

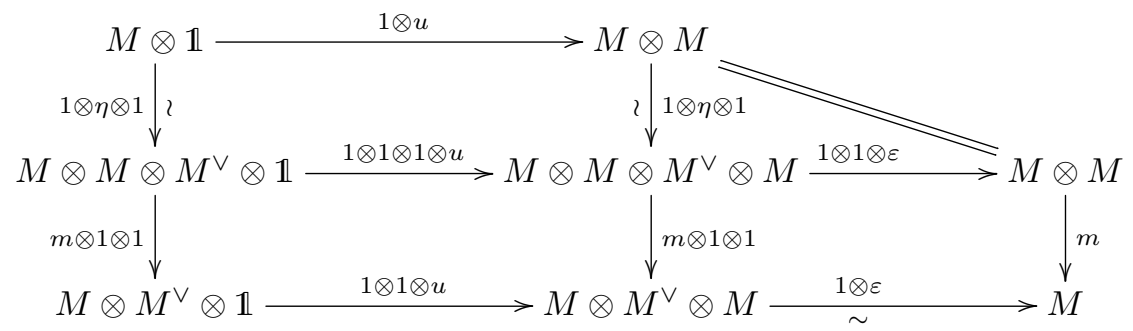

The clockwise composition is the identity and hence so is the counter-clockwise composition. It follows that the map

$$
M \otimes M^{\vee} \otimes \mathbb{1} \stackrel{1 \otimes 1 \otimes u}{\longrightarrow} M \otimes M^{\vee} \otimes M
$$


admits a right inverse. Since the functor $M \otimes M^{\vee} \otimes(-)$ is an equivalence, it follows that $u$ admits a right inverse.

We can now state and prove the following "Semiadditive Redshift" result, which can be informally summarized as "ht $=\mathrm{Ht}+1$ ".

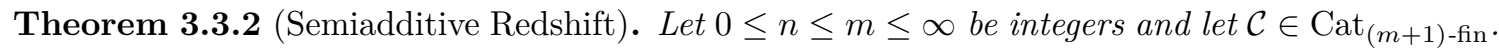
If $\mathcal{C}$ is p-typically m-semiadditive, then

(1) $\operatorname{Ht}(\mathcal{C}) \leq n$ if and only if $\operatorname{ht}(\mathcal{C}) \leq n+1$

(2) $\operatorname{Ht}(\mathcal{C})>n$ if and only if $\mathrm{ht}(\mathcal{C})>n+1$

In particular, an $\infty$-semiadditive $\infty$-category is of height $n$ if and only if, as an object of Cat $\infty$-fin (and hence also $\mathrm{Cat}^{\oplus-\infty}$ ), it is of height $n+1$.

Proof. Denote $B=B^{n+1} C_{p}$. For $(1)$, we observe that $\operatorname{Ht}(\mathcal{C}) \leq n$ if and only if $B$ is $\mathcal{C}$-acyclic (Proposition 3.2.1), namely that $B^{*}$ is fully faithful. On the other hand, the natural endomorphism $p_{(n+1)}=|B|$ of the identity functor of $\operatorname{Cat}_{(m+1) \text {-fin }}$, acts on $\mathcal{C}$ by the functor $B_{!} B^{*}: \mathcal{C} \rightarrow \mathcal{C}($ Example 2.2.8). Thus, ht $(\mathcal{C}) \leq n+1$, if and only if $B_{!} B^{*}$ is invertible. Now, if $B^{*}$ is fully faithful, then $B_{!} B^{*}=\operatorname{Id}_{\mathcal{C}}$ and is in particular invertible. On the other hand, if $B_{!} B^{*}$ is invertible, then $B^{*}$ is fully faithful by the dual of Lemma 3.3.1. Thus, $\operatorname{Ht}(\mathcal{C}) \leq n$ if and only if $\operatorname{ht}(\mathcal{C}) \leq n+1$.

For $(2)$, we first assume that $\operatorname{Ht}(\mathcal{C})>n$, and show that $\operatorname{ht}(\mathcal{C})>n+1$. Given $\mathcal{D} \in \operatorname{Cat}_{(m+1) \text {-fin }} \operatorname{such}$ that $\operatorname{ht}(\mathcal{D}) \leq n+1$, we need to show that every $(m+1)$-finite colimit preserving functor $F: \mathcal{D} \rightarrow \mathcal{C}$ must be zero. For every $X \in \mathcal{D}$, we have $X[B]=B_{!} B^{*} X \stackrel{\sim}{\longrightarrow} X$. Since $F$ is $(m+1)$-finite colimit preserving, we get also $F(X)[B] \stackrel{\sim}{\longrightarrow} F(X)$. Thus, by Proposition 2.4.7, we get $\operatorname{ht}(F(X)) \leq n$. Since $\operatorname{Ht}(\mathcal{C})>n$, the only object of height $\leq n$ in $\mathcal{C}$ is zero and hence $F(X)=0$. Thus, $F$ is zero, which proves that $\mathrm{ht}(\mathcal{C})>n+1$. Conversely, assume $\mathrm{ht}(\mathcal{C})>n+1$, to show that $\operatorname{Ht}(\mathcal{C})>n$, consider the full subcategory $\mathcal{C}_{\leq n} \subseteq \mathcal{C}$ spanned by objects of height $\leq n$ in $\mathcal{C}$, which is also $p$-typically $m$ semiadditive (Proposition 3.1.12). By definition, $\operatorname{Ht}\left(\mathcal{C}_{\leq n}\right) \leq n$, and hence by $(1), \operatorname{ht}\left(\mathcal{C}_{\leq n}\right) \leq n+1$, so the inclusion functor $\mathcal{C}_{\leq n} \hookrightarrow \mathcal{C}$ must be zero. It follows that $\mathcal{C}_{\leq n}$ is zero and therefore $\operatorname{Ht}(\mathcal{C})>n$. It follows from $(1)$ and $(2)$ that if $\operatorname{Ht}(\mathcal{C})=n$, then $\operatorname{ht}(\mathcal{C})=n+1$ when $\mathcal{C}$ is considered as an object of $\mathrm{Cat}_{\infty \text {-fin. }}$ The parenthetical remark follows from Proposition 3.1.15 and Proposition 2.2.11.

Recall from Definition 2.2.10, that for every $\mathcal{C} \in$ Cat $^{\oplus-m}$, the space of objects $\mathcal{C} \simeq$ is endowed with an $m$-commutative coCartesian monoid structure making it an object of the $m$-semiadditive $\infty$-category $\mathrm{CMon}_{m}$. Theorem 3.3.2 has the following corollary:

Corollary 3.3.3. Let $\mathcal{C} \in \mathrm{Cat}^{\oplus-\infty}$, such that $\operatorname{Ht}(\mathcal{C}) \leq n$. The space of objects $\mathcal{C} \simeq$ with the higher coCartesian structure satisfies $\mathrm{ht}\left(\mathcal{C}^{\simeq}\right) \leq n+1$, as an object of $\mathrm{CMon}_{\infty}$.

Proof. We have seen in Theorem 3.3.2, that when we consider $\mathcal{C}$ as an object of $\mathrm{Cat}_{\infty \text {-fin }}$, we have $\operatorname{ht}(\mathcal{C}) \leq n+1$. The space of objects $\mathcal{C} \simeq \in \mathrm{CMon}_{\infty}$ can be identified with the image of $\mathcal{C}$ under the $\infty$-semiadditive functor

$$
\operatorname{hom}\left(\mathcal{S}_{\infty \text {-fin }},-\right): \mathrm{Cat}_{\infty \text {-fin }} \rightarrow \mathrm{CMon}_{\infty} .
$$

Thus, by Proposition 3.1.13, we have $\operatorname{ht}\left(\mathcal{C}^{\simeq}\right) \leq n+1$. 
Example 3.3.4. Let $R$ be a $T(n)$-local ring spectrum. By Corollary 4.4 .3 and Theorem 4.4.5, the $\infty$-commutative monoid $\operatorname{Mod}_{R}\left(\operatorname{Sp}_{T(n)}\right) \simeq$ is of height $\leq n+1$ in $\mathrm{CMon}_{\infty}$. In particular, this applies to Morava $E$-theory $R=E_{n}$. This suggests a relation between the "semiadditive redshift" of Theorem 3.3.2 and the "chromatic redshift" in algebraic $K$-theory of Ausani-Rognes (see [AR08, AKQ19]). We shall explore this connection further in a future work.

The proof of Theorem 3.3.2 relies ultimately on the fact that $B^{n} C_{p}$ is $\mathcal{C}$-amenable if and only if $B^{n+1} C_{p}$ is $\mathcal{C}$-acyclic (Corollary 2.4.8). In Proposition 2.4.11 we categorified this fact by showing that $B^{n+1} C_{p}$ is $\mathcal{C}$-acyclic, if and only if $B^{n+2} C_{p}$ is $\mathcal{C}$-trivial. Similarly, Theorem 3.3 .2 can be categorified as follows. Let $\mathrm{Cat}^{n \text {-ht }} \subseteq$ Cat $_{\infty \text {-fin }}$ be the full subcategory spanned by the $\infty$-semiadditive $\infty$ categories $\mathcal{C}$ such that $\operatorname{Ht}(\mathcal{C})=n$.

Lemma 3.3.5. The full subcategory $\mathrm{Cat}^{n \text {-ht }} \subseteq$ Cat $_{\infty}$-fin is closed under $\pi$-finite colimits.

Proof. We have

$$
\mathrm{Cat}^{n-\mathrm{ht}} \subseteq \mathrm{Cat}^{\oplus-\infty} \subseteq \mathrm{Cat}_{\infty-\mathrm{fin}}
$$

By Theorem 3.3.2, we have

$$
\mathrm{Cat}^{n-\mathrm{ht}}=\left(\mathrm{Cat}^{\oplus-\infty}\right)_{n+1}
$$

Thus, by Proposition 3.1.12, Cat $^{n-\text { ht }}$ is closed under limits in Cat $^{\oplus-\infty}$. Additionally, Cat $^{\oplus-\infty}$ is closed under limits in Cat ${ }_{\infty}$-fin by Proposition 2.2.11. Therefore, Cat $^{n-h t}$ is closed under limits

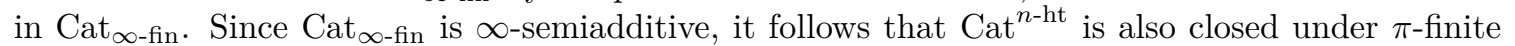
colimits in $\mathrm{Cat}_{\infty \text {-fin }}$.

Hence, in particular, Cat ${ }^{n \text {-ht }}$ admits $\pi$-finite colimits and is therefore an object of the $\infty$-semiadditive $\infty$-category $\widehat{\mathrm{Cat}}_{\infty \text {-fin }}$ of large $\infty$-categories, which admit $\pi$-finite colimits and functors preserving them.

Proposition 3.3.6. The $\infty$-category $\mathrm{Cat}^{n \text {-ht }}$ is an object of height $n+2$ in $\widehat{\mathrm{Cat}}_{\infty \text {-fin }}$.

Proof. By Lemma 3.3.5, Cat ${ }^{n \text {-ht }}$ is closed under $\pi$-finite colimits in Cat $_{\infty}$-fin and hence is $\infty$ semiadditive by Proposition 2.1.4. Thus, by Theorem 3.3.2, it suffices to show that for every $\mathcal{C} \in \mathrm{Cat}^{n \text {-ht }}$ we have ht $(\mathcal{C})=n+1$ as an object of Cat ${ }_{\infty \text {-fin }}$, and hence also as an object of Cat ${ }^{n \text {-ht }}$ (Proposition 3.1.15). This follows again from Theorem 3.3.2 and the fact that $\operatorname{Ht}(\mathcal{C})=n$.

\section{Stability}

So far, we have been considering general higher semiadditive $\infty$-categories. In this section, we specialize to the stable world. First, using the general results on height from the previous section, we shall show that every stable higher semiadditive $\infty$-category decomposes completely according to height. Second, inspired by [Lurb], we study semisimplicity properties of local systems valued in general stable $\infty$-categories of semiadditive height $n$. Finally, we show that $\operatorname{Sp}_{K(n)}$ and $\operatorname{Sp}_{T(n)}$ are indeed of semiadditive height $n$. 


\subsection{Recollement}

A central tool, which will be used several times in the following subsections, is that of a recollement of stable $\infty$-categories following [BG16]. In this preliminary subsection, we collect several general facts regarding this notion. First, we provide criteria for a recollement to be split, in the sense that it has trivial "gluing data". Second, we show that a decreasing intersection of a chain of recollements is again a recollement. Finally, we give special attention to recollements arising from "divisible" and "complete" objects with respect to a natural endomorphism of the identity functor in the sense of Definition 3.1.5.

\section{(Split) Recollement}

We begin by recalling the notion of recollement in the context of stable $\infty$-categories following the exposition in [Lura, Section A.8.1].

Definition 4.1.1. Let $\mathcal{C}$ be a stable $\infty$-category and $\mathcal{C}_{\circ} \subseteq \mathcal{C}$ a full stable subcategory. We define the right orthogonal complement $\mathcal{C}_{\circ}^{\perp} \subseteq \mathcal{C}$ to be the full subcategory consisting of objects $Y \in \mathcal{C}$, such that $\operatorname{Map}(X, Y) \simeq$ pt for all $X \in \mathcal{C}_{\circ}$.

Recall from [Lura, Proposition A.8.20], that if the inclusion $\mathcal{C}_{\circ} \hookrightarrow \mathcal{C}$ admits both a left adjoint $L$ and a right adjoint $R$, then $\mathcal{C}$ is a recollement of $\mathcal{C}_{\circ}$ and $\mathcal{C}_{\circ}^{\perp}$ in the sense of [Lura, Section A.8.1]. In particular, the $\infty$-category $\mathcal{C}$ can be identified with the $\infty$-category of sections of the cartesian fibration over $\Delta^{1}$, classified by the functor $\left.L\right|_{\mathcal{C}_{\circ}^{\perp}}: \mathcal{C}_{\circ}^{\perp} \rightarrow \mathcal{C}_{\circ}$.

Definition 4.1.2. We shall say that an inclusion of stable $\infty$-categories $\mathcal{C}_{\circ} \hookrightarrow \mathcal{C}$, that admits both a left and a right adjoint, exhibits $\mathcal{C}$ as a recollement of $\mathcal{C}_{\circ}$ and $\mathcal{C}_{\circ}^{\perp}$.

Recollements in stable homotopy theory typically arise from smashing localizations, and amount to the existence of various fracture squares:

Example 4.1.3 (Arithmetic and chromatic squares). For $\mathcal{C}=\operatorname{Sp}_{(p)}$, the inclusion of the full subcategory $\mathcal{C}_{\circ}=\operatorname{Sp}_{\mathbb{Q}} \subseteq \operatorname{Sp}_{(p)}$ admits both a left and a right adjoint, and we have

$$
\operatorname{Sp}_{\mathbb{Q}}^{\perp}=\widehat{\operatorname{Sp}}_{p} \subseteq \operatorname{Sp}_{(p)},
$$

is the full subcategory spanned by $p$-complete spectra. The recollement statement in this case recovers the classical $p$-local arithmetic square for spectra. More generally, the full subcategory

$$
L_{n}^{f} \mathrm{Sp}:=\mathrm{Sp}_{\oplus_{k=0}^{n}} T(k) \subseteq \mathrm{Sp}_{(p)}
$$

exhibits $\mathrm{Sp}_{(p)}$ as a recollement of $L_{n}^{f} \mathrm{Sp}$ and $\mathrm{Sp}_{F(n+1)}$, where $F(n+1)$ is a finite spectrum of type $n+1$. In particular, the inclusion $L_{n-1}^{f} \mathrm{Sp} \subseteq L_{n}^{f}$ Sp exhibits $L_{n}^{f}$ Sp as a recollement of $L_{n-1}^{f} \operatorname{Sp}$ and $\mathrm{Sp}_{T(n)}$. This recovers the classical telescopic fracture square at height $n$. By the Smash Product Theorem,

$$
L_{n} \mathrm{Sp}:=\operatorname{Sp}_{\oplus_{k=0}^{n} K(k)} \subseteq \mathrm{Sp}_{(p)}
$$

is also a smashing localization, and similarly, the inclusion $L_{n-1} \mathrm{Sp} \subseteq L_{n} \mathrm{Sp}$ exhibits $L_{n} \mathrm{Sp}$ as a recollement of $L_{n-1} \mathrm{Sp}$ and $\mathrm{Sp}_{K(n)}$. This recovers the classical chromatic fracture square at height $n$. 
Given a recollement $\mathcal{C}_{\circ} \subseteq \mathcal{C}$, the functor $\left.L\right|_{\mathcal{C}_{\circ}^{\perp}}: \mathcal{C}_{\circ}^{\perp} \rightarrow \mathcal{C}_{\circ}$ encodes the "gluing data" in the construction of $\mathcal{C}$ from the $\infty$-categories $\mathcal{C}_{\circ}$ and $\mathcal{C}_{\circ}^{\perp}$. A particularly simple instance of a recollement is when this gluing data is trivial:

Proposition 4.1.4. Given a recollement $\mathcal{C} \circ \subseteq \mathcal{C}$, the following are equivalent:

(1) The functor $\left.L\right|_{\mathcal{C}_{\circ}^{\perp}}: \mathcal{C}_{\circ}^{\perp} \rightarrow \mathcal{C}_{\circ}$ is zero.

(2) The left adjoints of the inclusions $\mathcal{C}_{\circ}, \mathcal{C}_{\circ}^{\perp} \subseteq \mathcal{C}$ induce an equivalence $\mathcal{C} \stackrel{\sim}{\longrightarrow} \mathcal{C}_{\circ} \times \mathcal{C}_{\circ}^{\perp}$.

(3) The left and right adjoints $L$ and $R$ of $\mathcal{C}_{\circ} \hookrightarrow \mathcal{C}$ are isomorphic.

Proof. We prove $(1) \Longrightarrow(2) \Longrightarrow(3) \Longrightarrow(1)$. The implication (1) $\Longrightarrow(2)$ follows from the identification of $\mathcal{C}$ with the $\infty$-category of sections of the cartesian fibration classified by $\left.L\right|_{\mathcal{C}_{\circ}^{\perp}}$. For the implication $(2) \Longrightarrow(3)$, observe that the inclusion $\mathcal{C}_{\circ} \hookrightarrow \mathcal{C}_{\circ} \times \mathcal{C}_{\circ}^{\perp}$ can be identified with the functor (Id, 0), for which the projection $\mathcal{C}_{\circ} \times \mathcal{C}_{\circ}^{\perp} \rightarrow \mathcal{C}_{\circ}$ is both a left and a right adjoint. Finally, for every $X \in \mathcal{C}_{\circ}^{\perp}$, we have $R(X)=0$. Thus, assuming (3) we have $R \simeq L$ and so $\left.L\right|_{\mathcal{C}_{\circ}^{\perp}}=0$, which proves (1).

Definition 4.1.5. We say that a recollement $\mathcal{C}_{\circ} \hookrightarrow \mathcal{C}$ is split if it satisfies the equivalent conditions of Proposition 4.1.4.

\section{Recollement chains}

We shall now study the behavior of chains of recollements.

Definition 4.1.6. For $\mathcal{C} \in \mathrm{Cat}_{\text {st }}$, we say that a descending chain of full subcategories,

$$
\cdots \subseteq \mathcal{C}_{(n)} \subseteq \cdots \subseteq \mathcal{C}_{(2)} \subseteq \mathcal{C}_{(1)} \subseteq \mathcal{C}_{(0)} \subseteq \mathcal{C}
$$

is a recollement chain, if each inclusion $\mathcal{C}_{(n)} \subseteq \mathcal{C}$ exhibits $\mathcal{C}$ as a recollement of $\mathcal{C}_{(n)}$ and $\mathcal{C}_{(n)}^{\perp}$. We also set

$$
\mathcal{C}_{(\infty)}:=\bigcap_{n \in \mathbb{N}} \mathcal{C}_{(n)}
$$

It turns out that under mild conditions, $\mathcal{C}_{(\infty)} \subseteq \mathcal{C}$ is itself a recollement.

Lemma 4.1.7. Let $\mathcal{C} \in \mathrm{Cat}_{\mathrm{st}}$, which admits sequential limits and colimits. For a recollement chain $\cdots \subseteq \mathcal{C}_{(2)} \subseteq \mathcal{C}_{(1)} \subseteq \mathcal{C}_{(0)} \subseteq \mathcal{C}$, the inclusion $\mathcal{C}_{(\infty)} \subseteq \mathcal{C}$ exhibits $\mathcal{C}$ as a recollement of $\mathcal{C}_{(\infty)}$ and $\mathcal{C}_{(\infty)}^{\perp}$.

Proof. It suffices to show that the inclusion $\mathcal{C}_{(\infty)} \hookrightarrow \mathcal{C}$ admits a left and a right adjoint. By symmetry, it suffices to consider only the left adjoint. By [Lur09, Proposition 5.2.7.8], for every $X \in \mathcal{C}$ we need to construct an object $L_{\infty} X \in \mathcal{C}_{(\infty)}$ and a morphism $X \stackrel{\eta}{\rightarrow} L_{\infty} X$, such that for all $Y \in \mathcal{C}_{(\infty)}$, the map

$$
\operatorname{Map}\left(L_{\infty} X, Y\right) \stackrel{(-) \circ \eta}{\longrightarrow} \operatorname{Map}(X, Y)
$$

is an isomorphism. Let $L_{n}: \mathcal{C} \rightarrow \mathcal{C}_{(n)}$ be the left adjoint of the inclusion $\mathcal{C}_{(n)} \hookrightarrow \mathcal{C}$ and denote by Id $\stackrel{\eta_{n}}{\longrightarrow} L_{n}$ the corresponding unit (i.e. localization) map, where we suppress the embedding functor 
$\mathcal{C}_{(n)} \subseteq \mathcal{C}$. Since the $\mathcal{C}_{(n)}$-s are nested, we have canonical isomorphisms $L_{n} L_{n-1} \stackrel{\sim}{\longrightarrow} L_{n}$, and we abuse notation by denoting the composition $L_{n-1} \stackrel{\eta_{n}}{\longrightarrow} L_{n} L_{n-1} \stackrel{\sim}{\longrightarrow} L_{n}$ also by $\eta_{n}$. We now define

$$
L_{\infty} X:=\stackrel{\lim _{\longrightarrow}}{\longrightarrow}\left(X \stackrel{\eta_{1}}{\longrightarrow} L_{1} X \stackrel{\eta_{2}}{\longrightarrow} L_{2} X \stackrel{\eta_{3}}{\longrightarrow} \ldots\right)
$$

and take $X \stackrel{\eta}{\rightarrow} L_{\infty} X$ to be the cone map from the first object to the colimit in the diagram defining $L_{\infty} X$. For every $Y \in \mathcal{C}_{(\infty)} \subseteq \mathcal{C}_{(n)}$, the map

$$
\operatorname{Map}\left(L_{n} X, Y\right) \stackrel{(-) \circ \eta_{n}}{\longrightarrow} \operatorname{Map}(X, Y)
$$

is an isomorphism for each $n \in \mathbb{N}$. Thus, by taking the limit over $n$, we get an isomorphism

$$
\lim _{\mathbb{N}} \operatorname{Map}\left(L_{n} X, Y\right) \stackrel{\sim}{\longrightarrow} \lim _{\mathbb{N}} \operatorname{Map}(X, Y) \simeq \operatorname{Map}(X, Y) .
$$

Precomposing with the isomorphism

$$
\operatorname{Map}\left(L_{\infty} X, Y\right)=\operatorname{Map}\left(\varliminf_{\mathbb{1}} L_{n} X, Y\right) \stackrel{\sim}{\longrightarrow} \varliminf_{\longleftarrow} \operatorname{Nap}\left(L_{n} X, Y\right),
$$

we get an isomorphism

$$
\operatorname{Map}\left(L_{\infty} X, Y\right) \stackrel{\sim}{\longrightarrow} \operatorname{Map}(X, Y) .
$$

Unwinding the definitions, this isomorphism is given by precomposition with $\eta$.

To identify the right orthogonal complement of $\mathcal{C}_{(\infty)}$ in $\mathcal{C}$, we need the following general categorical fact:

Lemma 4.1.8. Let $F: \mathcal{C} \rightarrow \mathcal{D}$ be a functor in $\mathrm{Cat}_{\mathrm{st}}$, and denote by $\operatorname{ker}(F) \subseteq \mathcal{C}$ the full subcategory spanned by the objects $X$, for which $F(X)=0$. If $F$ admits a fully faithful right adjoint $G: \mathcal{D} \hookrightarrow \mathcal{C}$, then $\operatorname{Im}(G)=\operatorname{ker}(F)^{\perp}$.

Proof. In one direction, for $X \in \operatorname{Im}(G)$, we have $X=G(Y)$ for some $Y \in \mathcal{D}$. Hence, for every $Z \in \operatorname{ker}(F)$ we have

$$
\operatorname{Map}(Z, X) \simeq \operatorname{Map}(Z, G(Y)) \simeq \operatorname{Map}(F(Z), Y) \simeq \operatorname{Map}(0, Y) \simeq \mathrm{pt} .
$$

Thus, $\operatorname{Im}(G) \subseteq \operatorname{ker}(F)^{\perp}$. Conversely, let $\operatorname{Id} \stackrel{u}{\rightarrow} G F$ and $F G \stackrel{c}{\rightarrow}$ Id be the unit and counit of the adjunction respectively. Since $G$ is fully faithful, $c$ is an isomorphism. By the zig-zag identities and 2-out-of-3, the map $F(u)$ is also an isomorphism. Now, for every $X \in \mathcal{C}$ consider the fiber sequence

$$
X_{0} \rightarrow X \stackrel{u}{\rightarrow} G F(X) .
$$

On the one hand, since $F(u)$ is an isomorphism, $F\left(X_{0}\right)=0$ and hence $X_{0} \in \operatorname{ker}(F)$. On the other hand, if $X \in \operatorname{ker}(F)^{\perp}$, then since $G F(X) \in \operatorname{Im}(G) \subseteq \operatorname{ker}(F)^{\perp}$, we also have $X_{0} \in \operatorname{ker}(F)^{\perp}$ and thus $X_{0}=0$. This implies that $u$ is an isomorphism and so $X \simeq G F(X) \in \operatorname{Im}(G)$.

Given a recollement chain as in Definition 4.1.6, for each $n \in \mathbb{N}$, we have a fully faithful embedding $\mathcal{C}_{(n)}^{\perp} \hookrightarrow \mathcal{C}$ with left adjoint $P_{n}: \mathcal{C} \rightarrow \mathcal{C}_{(n)}^{\perp}$. We abuse notation by suppressing the inclusion $\mathcal{C}_{n}^{\perp} \subseteq \mathcal{C}$ and the canonical isomorphisms $P_{n} P_{n+1} \simeq P_{n}$. We thus obtain a tower

$$
\ldots \stackrel{P_{n+1}}{\longrightarrow} \mathcal{C}_{(n)}^{\perp} \stackrel{P_{n}}{\longrightarrow} \ldots \stackrel{P_{2}}{\longrightarrow} \mathcal{C}_{(2)}^{\perp} \stackrel{P_{1}}{\longrightarrow} \mathcal{C}_{(1)}^{\perp} \stackrel{P_{0}}{\longrightarrow} \mathcal{C}_{(0)}^{\perp}
$$

of $\infty$-categories under $\mathcal{C}$, which induces a functor

$$
P_{\infty}: \mathcal{C} \rightarrow \lim _{\longleftarrow} \mathcal{C}_{(n)}^{\perp} .
$$


Proposition 4.1.9. Let $\mathcal{C} \in \mathrm{Cat}_{\text {st }}$ which admits sequential limits and colimits. Given a recollement chain $\cdots \subseteq \mathcal{C}_{(2)} \subseteq \mathcal{C}_{(1)} \subseteq \mathcal{C}_{(0)} \subseteq \mathcal{C}$, the functor $P_{\infty}: \mathcal{C} \rightarrow \varliminf_{\varlimsup} \mathcal{C}_{(n)}^{\perp}$ admits a fully faithful right adjoint, whose essential image is $\mathcal{C}_{(\infty)}^{\perp}$. Thus, $\mathcal{C}$ is a recollement of $\mathcal{C}_{(\infty)}$ and $\varliminf_{\longleftarrow} \mathcal{C}_{(n)}^{\perp}$.

Proof. By Lemma 4.1.7, the inclusion $\mathcal{C}_{(\infty)} \hookrightarrow \mathcal{C}$ exhibits $\mathcal{C}$ as a recollement of $\mathcal{C}_{(\infty)}$ and its right orthogonal complement and hence it suffices to identify $\mathcal{C}_{(\infty)}^{\perp}$. The objects of $\mathcal{C}_{(\infty)}$ are precisely the $X \in \mathcal{C}$ for which $P_{\infty}(X)=0$. Thus, by Lemma 4.1.8, it suffices to show that $P_{\infty}$ admits a fully faithful right adjoint. Since $P_{n}$ is a left adjoint for all $n$, by [HY17, Theorem B], the functor $P_{\infty}$ is a left adjoint and we denote its right adjoint by $G_{\infty}: \lim _{\leftarrow} \mathcal{C}_{(n)}^{\perp} \rightarrow \mathcal{C}$. We show that $G_{\infty}$ is fully faithful using the explicit description of the adjunction $P_{\infty} \dashv G_{\infty}$ given in [HY17]. An object of $\lim _{\longrightarrow} \mathcal{C}_{(n)}^{\perp}$ consists of a sequence of objects $X_{n} \in \mathcal{C}_{(n)}^{\perp}$ together with structure isomorphisms $P_{n} X_{n+1} \stackrel{\sim}{\longrightarrow} X_{n}$. We shall write $\left\{X_{n}\right\} \in \lim _{\longleftarrow} \mathcal{C}_{(n)}^{\perp}$ suppressing the structure isomorphisms. Composing the structure isomorphisms of $\left\{X_{n}\right\}$ with the corresponding unit (i.e. localization) maps Id $\stackrel{u_{n}}{\longrightarrow} P_{n}$, we get maps as follows:

$$
f_{n}: X_{n+1} \stackrel{u_{n}}{\longrightarrow} P_{n} X_{n+1} \stackrel{\sim}{\longrightarrow} X_{n} .
$$

By [HY17, Theorem B], the functor $G_{\infty}$ can be described explicitly on objects by the following formula:

$$
G_{\infty}\left(\left\{X_{n}\right\}\right) \simeq \lim _{\longleftarrow}\left(\ldots \stackrel{f_{n}}{\longrightarrow} X_{n} \stackrel{f_{n-1}}{\longrightarrow} \ldots \stackrel{f_{2}}{\longrightarrow} X_{2} \stackrel{f_{1}}{\longrightarrow} X_{1} \stackrel{f_{0}}{\longrightarrow} X_{0}\right) .
$$

To prove that $G_{\infty}$ is fully faithful, it suffices to show that the counit $P_{\infty} G_{\infty} \stackrel{c}{\rightarrow}$ Id is an isomorphism. Since the collection of projection functors $\pi_{k}: \lim _{\longleftarrow} \mathcal{C}_{(n)}^{\perp} \rightarrow \mathcal{C}_{(k)}^{\perp}$ for all $k \in \mathbb{N}$ is jointly conservative, it suffices to show that

$$
P_{k}\left(\lim _{\longleftarrow} X_{n}\right) \stackrel{\pi_{k}(c)}{\longrightarrow} X_{k}
$$

is an isomorphism for all $k \in \mathbb{N}$ and $\left\{X_{n}\right\} \in \lim _{\longleftarrow} \mathcal{C}_{(n)}^{\perp}$. By [HY17, Theorem 5.5], we can describe $\pi_{k}(c)$ as the composition

$$
P_{k}\left(\varliminf_{i} X_{n}\right) \rightarrow P_{k}\left(X_{k}\right) \stackrel{\sim}{\longrightarrow} X_{k},
$$

where the first map is induced by the canonical projection $\lim _{\longleftarrow} X_{n} \rightarrow X_{k}$. By cofinality, we can assume that the limit is taken over $n \geq k$. By definition, for each $n \in \mathbb{N}$, the fiber of $X_{n+1} \stackrel{f_{n}}{\longrightarrow} X_{n}$ lies in $\mathcal{C}_{(n)} \subseteq \mathcal{C}_{(k)}$. Since $\mathcal{C}_{(k)}$ is closed under sequential limits, it follows that the fiber of $\lim _{n} X_{n} \rightarrow X_{k}$ lies in $\mathcal{C}_{(k)}$ and hence becomes an isomorphism after applying $P_{k}$. This concludes the proof that $G_{\infty}$ is fully faithful and hence the proof of the claim.

Corollary 4.1.10. Let $\mathcal{C} \in \mathrm{Cat}_{\mathrm{st}}$ which admits sequential limits and colimits with a recollement chain $\cdots \subseteq \mathcal{C}_{(2)} \subseteq \mathcal{C}_{(1)} \subseteq \mathcal{C}_{(0)} \subseteq \mathcal{C}$. If $\mathcal{C}_{(\infty)}=0$ then $\mathcal{C} \simeq \lim _{\longleftarrow} \mathcal{C}_{(n)}^{\perp}$.

Proof. By Proposition 4.1.9, $\mathcal{C}$ is a recollement of $\mathcal{C}_{(\infty)}=0$ and of $\lim _{\longleftarrow} \mathcal{C}_{(n)}^{\perp}$, so that $\mathcal{C} \simeq \lim _{\longleftarrow} \mathcal{C}_{(n)}^{\perp}$.

\section{Divisible and complete recollement}

One way to get a recollement is by taking the divisible and complete objects with respect to a natural endomorphism of the identity functor. That is, given a stable $\infty$-category $\mathcal{C}$ and $\operatorname{Id}_{\mathcal{C}} \stackrel{\alpha}{\rightarrow} \operatorname{Id}_{\mathcal{C}}$, we have the full subcategories $\mathcal{C}\left[\alpha^{-1}\right]$ and $\widehat{\mathcal{C}}_{\alpha}=\mathcal{C}\left[\alpha^{-1}\right]^{\perp}$ of $\mathcal{C}$ (Definition 3.1.5). Assuming $\mathcal{C}$ admits 
sequential limits and colimits, the inclusion $\mathcal{C}\left[\alpha^{-1}\right] \hookrightarrow \mathcal{C}$ admits both a left adjoint $L$ and a right adjoint $R$, given respectively by "inverting $\alpha$ " on $X$

$$
L X=\lim _{\longrightarrow}(X \stackrel{\alpha}{\rightarrow} X \stackrel{\alpha}{\rightarrow} X \stackrel{\alpha}{\rightarrow} \ldots)
$$

and by taking the " $\alpha$-divisible part" of $X$

$$
R X=\lim _{\longleftarrow}(\ldots \stackrel{\alpha}{\rightarrow} X \stackrel{\alpha}{\rightarrow} X \stackrel{\alpha}{\rightarrow} X) .
$$

Remark 4.1.11. We warn the reader that although the above statements are well known and fairly intuitive, they are not as tautological as one might think. In particular, they might fail if $\mathcal{C}$ is not assumed to be stable (or at least additive). We refer the reader to [BNT18, Appendix C], for a comprehensive treatment of a closely related situation.

Note that an object $Y \in \mathcal{C}$ is $\alpha$-complete if and only if $R Y=0$ if and only if $Y \simeq \lim Y / \alpha^{r}$. In fact, the $\alpha$-completion functor

$$
Y \mapsto \widehat{Y}_{\alpha}:=\lim _{\longleftarrow} Y / \alpha^{r},
$$

is the left adjoint to the inclusion $\widehat{\mathcal{C}}_{\alpha} \hookrightarrow \mathcal{C}$.

Proposition 4.1.12. Let $\mathcal{C} \in \mathrm{Cat}_{\text {st }}$ which admits sequential limits and colimit and let $\operatorname{Id}_{\mathcal{C}} \stackrel{\alpha}{\rightarrow} \operatorname{Id}_{\mathcal{C}}$. Then $\mathcal{C}$ is a recollement of $\mathcal{C}\left[\alpha^{-1}\right]$ and $\widehat{\mathcal{C}_{\alpha}}$.

Proof. It follows from the discussion above that $\mathcal{C}\left[\alpha^{-1}\right] \hookrightarrow \mathcal{C}$ admits both adjoints.

Our next goal is to give a characterization of when the said recollement is split in terms of the natural endomorphism $\alpha$.

Definition 4.1.13. We say that a natural endomorphism $\beta: \operatorname{Id}_{\mathcal{C}} \rightarrow \operatorname{Id}_{\mathcal{C}}$ is a semi-inverse of $\alpha$, if for every $\alpha$-divisible $X$, the map $\beta_{X}$ is an inverse of $\alpha_{X}$.

The usefulness of the notion of semi-inverse is in that it allows us to characterize completeness in terms of divisibility:

Proposition 4.1.14. Let $\mathcal{C} \in \mathrm{Cat}_{\mathrm{st}}$ which admits sequential limits and colimits. For every $\alpha, \beta: \operatorname{Id}_{\mathcal{C}} \rightarrow \operatorname{Id}_{\mathcal{C}}$, if an object $Y \in \mathcal{C}$ is $\alpha$-complete, then it is $(1-\alpha \beta)$-divisible. If $\beta$ is a semi-inverse of $\alpha$, then the converse holds as well.

Proof. Note that all natural endomorphisms of $\operatorname{Id}_{\mathcal{C}}$ commute by the interchange law, so in particular $\alpha \beta=\beta \alpha$. For an $\alpha$-complete object $Y \in \mathcal{C}$ we have $Y=\lim _{\longleftarrow} Y / \alpha^{r}$. For every $r \in \mathbb{N}$, the map $\alpha^{2 r}$ is zero on $Y / \alpha^{r}$ and hence $1-\alpha \beta$ is invertible on $Y / \alpha^{r}$. By passing to the limit, $1-\alpha \beta$ is invertible on $Y$. Conversely, assume that $(1-\alpha \beta)$ acts invertibly on $Y$. If $\beta$ is a semi-inverse of $\alpha$, then for every $\alpha$-divisible $X$, the map $(1-\alpha \beta)$ acts as zero on $X$. Thus the pointed space $\operatorname{Map}(X, Y)$ must be contractible as $(1-\alpha \beta)$ acts both invertibly and as zero on it. This implies that $Y$ is $\alpha$-complete.

The above lemma leads us to the following characterization of split recollement: 
Proposition 4.1.15. Let $\mathcal{C}$ be a stable $\infty$-category which admits sequential limits and colimits. The recollement associated with a natural endomorphism $\operatorname{Id}_{\mathcal{C}} \stackrel{\alpha}{\rightarrow} \mathrm{Id}_{\mathcal{C}}$ is split if and only if $\alpha$ admits a semi-inverse. In which case,

$$
\mathcal{C} \simeq \mathcal{C}\left[\alpha^{-1}\right] \times \widehat{\mathcal{C}}_{\alpha}
$$

Proof. Let $\beta$ be a semi-inverse of $\alpha$. To show that $L Y=0$ for all $Y \in \widehat{\mathcal{C}}_{\alpha}$ it suffices to show that $\operatorname{Map}(Y, X)$ is contractible for all $X \in \mathcal{C}\left[\alpha^{-1}\right]$. By definition, $1-\alpha \beta$ is zero on $X$, so it suffices to observe that $1-\alpha \beta$ is invertible on $Y$ by Proposition 4.1.14. Conversely, if $\mathcal{C} \simeq \mathcal{C}\left[\alpha^{-1}\right] \times \widehat{\mathcal{C}_{\alpha}}$, we have for every $X \in \mathcal{C}$ a natural decomposition $X \simeq X\left[\alpha^{-1}\right] \oplus \widehat{X}_{\alpha}$ with $X\left[\alpha^{-1}\right] \in \mathcal{C}\left[\alpha^{-1}\right]$ and $\widehat{X}_{\alpha} \in \widehat{\mathcal{C}}_{\alpha}$. In this case, the map $\beta=\left(\left.\alpha\right|_{X\left[\alpha^{-1}\right]}\right)^{-1} \oplus 0_{\widehat{X}_{\alpha}}$ is a semi-inverse of $\alpha$.

Remark 4.1.16. In Example 4.1.3, the recollement $\mathrm{Sp}_{\mathbb{Q}} \subseteq \mathrm{Sp}_{(p)}$ corresponds to the endomorphism $\operatorname{Id}_{\mathrm{Sp}_{(p)}} \stackrel{p}{\rightarrow} \operatorname{Id}_{\mathrm{Sp}_{(p)}}$. However, not every recollement arises in such a way. For example, for $n \geq 1$ the recollement $L_{n} \mathrm{Sp} \subseteq \mathrm{Sp}_{(p)}$ is not induced by any endomorphism $\alpha$ of the identity functor. We do note however, that every split recollement $\mathcal{C}_{\circ} \subseteq \mathcal{C}$ must arise from an endomorphism $\alpha$ of $\operatorname{Id}_{\mathcal{C}}$, because we can take $\alpha$ to be the idempotent $\varepsilon: \operatorname{Id}_{\mathcal{C}} \rightarrow \operatorname{Id}_{\mathcal{C}}$ projecting onto $\mathcal{C}_{\circ}$. In this case, $\varepsilon$ itself is a semi-inverse of $\varepsilon$.

\subsection{Height Decomposition}

Let $\mathcal{C}$ be now a stable $m$-semiadditive $\infty$-category. We shall use the general machinery of (split) recollement to show that $\mathcal{C}$ splits into a product of $\infty$-categories according to height. By definition,

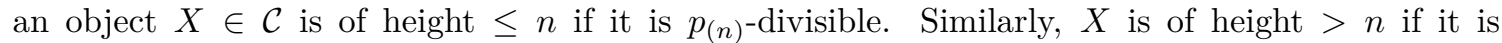
$p_{(n)}$-complete, which by Proposition 3.1.9, is if and only if it is complete with respect to all of $p=p_{(0)}, p_{(1)}, \ldots, p_{(n)}$. Accordingly,

Proposition 4.2.1. Let $\mathcal{C} \in \mathrm{Cat}_{\mathrm{st}}^{\oplus-m}$ and let $0 \leq n \leq m$. If $\mathcal{C}$ admits sequential limits and colimits, then $\mathcal{C}$ is a recollement of $\mathcal{C}_{\leq n}$ and $\mathcal{C}_{>n}$.

Proof. The full subcategory $\mathcal{C}_{\leq n} \subseteq \mathcal{C}$ consists of the $p_{(n)}$-divisible objects and $\mathcal{C}_{>n} \subseteq \mathcal{C}$ is the full subcategory of $p_{(n)}$-complete objects. Thus, the result follows from Proposition 4.1.12.

Our next goal is to show that under suitable assumptions, this recollement is in fact split. For this we need the following:

Proposition 4.2.2. Let $\mathcal{C} \in \mathrm{Cat}_{\mathrm{st}}^{\oplus-m}$ and assume it admits sequential limits and colimits. For all $n=0, \ldots, m-1$, the map $p_{(n+1)}$ is a semi-inverse of $p_{(n)}$. In particular, for $X \in \mathcal{C}$, we have $\operatorname{ht}(X)>n$ if and only if $X$ is $\left(1-p_{(n)} p_{(n+1)}\right)$-divisible.

Proof. If

$$
p_{(n)}=\left|B^{n} C_{p}\right|=\left|\Omega B^{n+1} C_{p}\right|
$$

is invertible on $X \in \mathcal{C}$, then by Proposition 2.4.7, $p_{(n+1)}=\left|B^{n+1} C_{p}\right|$ is the inverse of $p_{(n)}$ on $X$. Thus, $p_{(n+1)}$ is a semi-inverse of $p_{(n)}$. Therefore, the claim follows from Proposition 4.1.14.

Using Proposition 4.2.2 we can improve on Proposition 3.1.13 in the stable case as follows: 
Corollary 4.2.3. Let $F: \mathcal{C} \rightarrow \mathcal{D}$ be a functor in $\mathrm{Cat}^{\oplus-m}$ and assume $\mathcal{C}$ and $\mathcal{D}$ are stable. For every $X \in \mathcal{C}$ and $0 \leq n \leq m$, if $X$ is of height $\leq n$ or $>n-1$, then so is $F(X)$. The converse holds if $F$ is conservative.

Proof. The facts about height $\leq n$ follow from Proposition 3.1.13. The facts about height $>n-1$ follow similarly using Proposition 4.2.2. Namely, that $X$ is of height $>n-1$, if and only if $\left(1-p_{(n-1)} p_{(n)}\right)$ acts invertibly on it.

Remark 4.2.4. Corollary 4.2 .3 does not cover the case $h t(X)>m$. This indeed can not be guaranteed even in the stable case as witnessed by Example 3.1.14.

Similarly, we can improve on Corollary 3.1 .16 as follows:

Corollary 4.2.5. Let $\mathcal{C} \in \mathrm{Cat}_{\mathrm{st}}$ be $p$-typically $m$-semiadditively monoidal $\infty$-category. For every $0 \leq n \leq m$, we have $\operatorname{Ht}(\mathcal{C}) \leq n$ or $\operatorname{Ht}(\mathcal{C})>n-1$ if and only if $\operatorname{ht}_{\mathcal{C}}(\mathbb{1}) \leq n$ or $\operatorname{ht}_{\mathcal{C}}(\mathbb{1})>n-1$ respectively.

Proof. Given $X \in \mathcal{C}$, the functor $X \otimes(-): \mathcal{C} \rightarrow \mathcal{C}$ is $p$-typically $m$-semiadditive. Thus, the claim follows from Corollary 4.2.3.

Remark 4.2.6. Again, in Corollary 4.2 .5 it is not true that if $\operatorname{ht}(\mathbb{1})>m$ then $\operatorname{Ht}(\mathcal{C})>m$. For example, $\operatorname{Mod}_{\widehat{\mathbb{S}}_{p}}(\mathrm{Sp})$ is a presentably symmetric monoidal 0 -semiadditive $\infty$-category, whose unit $\widehat{\mathbb{S}}_{p}$ has height $>0$, although the $\infty$-category itself does not.

The following is our main structure theorem for stable higher semiadditive $\infty$-categories:

Theorem 4.2.7 (Height Decomposition). Let $\mathcal{C} \in \mathrm{Cat}_{\mathrm{st}}^{\oplus-m}$ for some $0 \leq m \leq \infty$.

(1) For $m<\infty$, and $\mathcal{C}$ which is idempotent complete, the inclusions $\mathcal{C}_{0}, \ldots, \mathcal{C}_{m-1}, \mathcal{C}_{>m-1} \subseteq \mathcal{C}$ determine an equivalence of $\infty$-categories

$$
\mathcal{C} \simeq \mathcal{C}_{0} \times \cdots \times \mathcal{C}_{m-1} \times \mathcal{C}_{>m-1}
$$

Moreover, $\mathcal{C}_{0}$ is p-typically $\infty$-semiadditive and $\mathcal{C}_{1}, \ldots, \mathcal{C}_{m-1}$ are $\infty$-semiadditive.

(2) For $m=\infty$, and $\mathcal{C}$ which admits sequential limits and colimits, $\mathcal{C}$ is a recollement of $\mathcal{C}_{\infty}$ and $\prod_{n \in \mathbb{N}} \mathcal{C}_{n}$. In particular, if $\mathcal{C}_{\infty}=0$, then $\mathcal{C} \simeq \prod_{n \in \mathbb{N}} \mathcal{C}_{n}$

If in addition $\mathcal{C}$ is $m$-semiadditively $\mathcal{O}$-monoidal for some $\infty$-operad $\mathcal{O}$, then $\mathcal{C}_{>m-1}$ and $\mathcal{C}_{n}$ for all $n=0, \ldots, m-1$, are compatible with the $\mathcal{O}$-monoidal structure and the equivalences in both (1) and (2) promote naturally to an equivalence of $\mathcal{O}$-monoidal $\infty$-categories.

Proof. (1) We first prove the claim under the additional assumption that $\mathcal{C}$ admits all sequential limits and colimits (and hence in particular idempotent complete). First, by Proposition 4.2.2, the map $p_{(m)}$ is a semi-inverse of $p_{(m-1)}$. Hence, by Proposition 4.1.15, we obtain a direct product decomposition $\mathcal{C} \simeq \mathcal{C}_{\leq m-1} \times \mathcal{C}_{>m-1}$. The category $\mathcal{C}_{\leq m-1}$ is itself $m$-semiadditive (Proposition 3.1.12) and admits all sequential limits and colimits. Thus, we can continue decomposing $\mathcal{C}_{\leq m-1}$ inductively and get $\mathcal{C}_{\leq n} \simeq \mathcal{C}_{\leq n-1} \times \mathcal{C}_{n}$ for all $n=0, \ldots, m-1$. Finally, by Proposition 3.2.2, each $\mathcal{C}_{\leq n}$ is in fact $p$-typically $\infty$-semiadditive. By Proposition $3.1 .12, \mathcal{C}_{n}$ is also $p$-typically $\infty$-semiadditive 
and since it is also $p$-complete for all $n \geq 1$, it is in particular $p$-local, and hence $\infty$-semiadditive by Proposition 3.2.6.

For a general $\mathcal{C}$ as in the claim, we use a semiadditive version of the Yoneda embedding to reduce to the presentable case. Namely, we shall show in Proposition 5.3.5, that there exists a presentable stable $m$-semiadditive $\infty$-category $\widehat{\mathcal{C}}$ and an $m$-semiadditive fully faithful embedding $\mathcal{C} \hookrightarrow \widehat{\mathcal{C}}$. By Corollary 4.2.3, for each $n=0, \ldots, m-1$, we have fully faithful embedding $\mathcal{C}_{n} \hookrightarrow \widehat{\mathcal{C}}_{n}$ and $\mathcal{C}_{>m-1} \hookrightarrow$ $\widehat{\mathcal{C}}_{>m-1}$, hence also

$$
\mathcal{C}_{0} \times \cdots \times \mathcal{C}_{m-1} \times \mathcal{C}_{>m-1} \hookrightarrow \widehat{\mathcal{C}}_{0} \times \cdots \times \widehat{\mathcal{C}}_{m-1} \times \widehat{\mathcal{C}}_{>m-1} \simeq \widehat{\mathcal{C}}
$$

By the left cancellation property of fully faithful embeddings, we get a fully faithful embedding

$$
\mathcal{C}_{0} \times \cdots \times \mathcal{C}_{m-1} \times \mathcal{C}_{>m-1} \hookrightarrow \mathcal{C} .
$$

For each object $X \in \mathcal{C}$, the height $n=0, \ldots, m-1$ and $>m-1$ components of $X$ in $\widehat{\mathcal{C}}$, are retracts of $X$ in $\widehat{\mathcal{C}}$. Thus, if $\mathcal{C}$ is idempotent complete, these components belong to $\mathcal{C}$. It follows that the above fully faithful embedding is also essentially surjective.

(2) For every $n<\infty$, we have by (1), that $\mathcal{C} \simeq \mathcal{C}_{\leq n} \times \mathcal{C}_{>n}$. Hence, we can switch the roles of $\mathcal{C}_{\leq n}$ and $\mathcal{C}_{>n}$, and consider the embedding $\mathcal{C}_{>n} \subseteq \mathcal{C}$ as exhibiting $\mathcal{C}$ as a recollement of $\mathcal{C}_{>n}$ and $\left(\overline{\mathcal{C}}_{>n}\right)^{\perp}=\mathcal{C}_{\leq n}$. We thus obtain a recollement chain

$$
\ldots \subseteq \mathcal{C}_{>2} \subseteq \mathcal{C}_{>1} \subseteq \mathcal{C}_{>0} \subseteq \mathcal{C}
$$

By definition, $\mathcal{C}_{\infty}=\bigcap_{n \in \mathbb{N}} \mathcal{C}_{>n}$, and so by Proposition 4.1.9, $\mathcal{C}$ is a recollement of $\mathcal{C}_{\infty}$ and

$$
\lim _{\swarrow} \ltimes \in \mathbb{N}\left(\mathcal{C}_{\leq n}\right) \simeq \lim _{n \in \mathbb{N}}\left(\prod_{0 \leq k \leq n} \mathcal{C}_{k}\right) \simeq \prod_{n \in \mathbb{N}} \mathcal{C}_{n} .
$$

Finally, assume that $\mathcal{C}$ is $m$-semiadditively $\mathcal{O}$-monoidal. The full subcategories $\mathcal{C}_{\leq n}$ and $\mathcal{C}_{>n}$ for all $n=0, \ldots, m-1$ consist of objects which are $p_{(n)}$-divisible and $\left(1-p_{(n)} p_{(n+1)}\right)$-divisible respectively. It follows that $\mathcal{C}_{\leq n}$ and $\mathcal{C}_{>n}$ are compatible with the $\mathcal{O}$-monoidal structure and hence so is $\mathcal{C}_{n}$ for all $n=0, \ldots, m-1$. Thus, by [Lura, Proposition 2.2.1.9], the $\mathcal{C}_{n}$-s and $\mathcal{C}_{>m-1}$ inherit an $\mathcal{O}$-monoidal structure such that the projections $\mathcal{C} \rightarrow \mathcal{C}_{n}$ and $\mathcal{C} \rightarrow \mathcal{C}_{>m-1}$ are $\mathcal{O}$-monoidal.

We conclude with some remarks regarding the sharpness of Theorem 4.2.7. In the case $m<\infty$, the fact that $\mathcal{C}$ is $m$-semiadditive also implies that $\mathcal{C}$ is a recollement of $\mathcal{C}_{\leq m}$ and $\mathcal{C}_{>m}$, but there is no guaranty that the "gluing data" is trivial. That is, that $\mathcal{C}$ decomposes as a direct product of $\mathcal{C}_{\leq m}$ and $\mathcal{C}_{>m}$. Indeed, consider the 0 -semiadditive $\infty$-category $\mathcal{C}=\operatorname{Sp}_{(p)}$. The case $m=0$ corresponds to the recollement $\mathrm{Sp}_{\mathbb{Q}} \subseteq \mathrm{Sp}_{(p)}$ of Example 4.1.3. In this case $\mathcal{C}_{>0}=\widehat{\mathrm{Sp}}_{p}$ and the gluing data is not trivial, as the rationalization of the $p$-completion does not vanish in general. Having said that, for $m \geq 1$ we do not know whether there even exists a stable $p$-local presentable $\infty$-category that is $m$-semiadditive, but not $(m+1)$-semiadditive [CSY18, Conjecture 1.1.5].

In the case $m=\infty$, we do not know whether there exists a stable $\infty$-semiadditive $\infty$-category $\mathcal{C}$ for which $\mathcal{C}_{\infty} \neq 0$. We hence propose the following: 
Conjecture 4.2.8 (Height Finiteness). For every $\mathcal{C} \in \operatorname{Pr}_{\mathrm{st}}^{\oplus-\infty}$, the full subcategory

$$
\mathcal{C}_{\infty}:=\bigcap_{n \geq 0} \mathcal{C}_{>n} \subseteq \mathcal{C}
$$

of objects of height $\infty$, is trivial.

\subsection{Semisimplicity}

Classical representation theory tells us that in characteristic 0 , representations of a finite group $G$ are semisimple. The $\infty$-category of $\mathcal{C}$-representations of $G$ in any $\infty$-category $\mathcal{C}$ is equivalent to the $\infty$-category $\mathcal{C}^{B G}$ of $\mathcal{C}$-valued local systems on $B G$. From the point of view of higher semiadditivity, characteristic 0 corresponds to semiadditive height 0 , and so it is natural to consider the analogous situation for higher heights. We shall show that given a stable $\infty$-semiadditive $\infty$-category $\mathcal{C}$ of height $n$, certain analogous semisimplicity phenomena hold for $\mathcal{C}^{A}$ for every $\pi$-finite $n$-connected space $A$ (e.g. $A=B^{n+1} G$ ). For the case $\mathcal{C}=\operatorname{Mod}_{K(n)}(\mathrm{Sp})$ these ideas were discussed in [Lurb].

\section{Splitting local systems}

The main result of this subsection is the following relation between acyclic maps and split recollement in the stable setting:

Proposition 4.3.1. Let $\mathcal{C} \in \mathrm{Cat}_{\mathrm{st}}$ and let $A \stackrel{q}{\rightarrow} B$ be a $\mathcal{C}$-acyclic and weakly $\mathcal{C}$-ambidextrous map. The functor $q^{*}: \mathcal{C}^{B} \rightarrow \mathcal{C}^{A}$ is fully faithful and exhibits $\mathcal{C}^{A}$ as a recollement of $\mathcal{C}^{B}$ and $\left(\mathcal{C}^{B}\right)^{\perp} \subseteq \mathcal{C}^{A}$. The recollement is split if and only if $q$ is $\mathcal{C}$-ambidextrous, in which case there is a canonical equivalence:

$$
\mathcal{C}^{A} \simeq \mathcal{C}^{B} \times\left(\mathcal{C}^{B}\right)^{\perp}
$$

Proof. By definition of acyclicity, $q^{*}$ is fully faithful. Hence, the recollement is split if and only the left and right adjoints $q$ ! and $q_{*}$ respectively of $q^{*}$ are isomorphic (Proposition 4.1.4). If $q$ is $\mathcal{C}$-ambidextrous then $q_{\text {! }}$ and $q_{*}$ are isomorphic and the recollement is split. Conversely, if we have $q_{!} \simeq q_{*}$, then $q_{*}$ preserves all $q$-colimits and hence $q$ is $\mathcal{C}$-ambidextrous (Proposition 2.1.3).

As a special case we obtain:

Theorem 4.3.2. Let $\mathcal{C} \in \mathrm{Cat}_{\mathrm{st}}^{\oplus-\infty}$ be $p$-local such that $\operatorname{Ht}(\mathcal{C})=n$. For every map of spaces $A \stackrel{q}{\rightarrow} B$ with $n$-connected $\pi$-finite fibers, we have a canonical equivalence

$$
\mathcal{C}^{A} \simeq \mathcal{C}^{B} \times\left(\mathcal{C}^{B}\right)^{\perp}
$$

Proof. Since $\mathcal{C}$ is of height $n$, the map $q$ is $\mathcal{C}$-acyclic by Theorem 3.2.7(2) and Proposition 2.4.2. Hence, the claim follows from Proposition 4.3.1.

In the case $B=\mathrm{pt}$, we can interpret Theorem 4.3.2 from the perspective of "higher representation theory" (see [Lurb]). For every space $A$, we call an object $X \in \mathcal{C}^{A}$ unipotent if it belongs to the full subcategory $\mathcal{C}_{\text {unip }}^{A} \subseteq \mathcal{C}^{A}$ generated by colimits from the trivial representations (i.e. constant 
local systems). It can be shown that every object $X \in \mathcal{C}^{A}$ fits into an essentially unique cofiber sequence

$$
X_{\text {unip }} \rightarrow X \rightarrow X^{0}
$$

with $X_{\text {unip }} \in \mathcal{C}_{\text {unip }}^{A}$ and $X^{0} \in\left(\mathcal{C}_{\text {unip }}^{A}\right)^{\perp}$. If $A$ is $n$-connected, then Theorem 4.3.2 implies that $X_{\text {unip }}$ is constant and $(*)$ canonically splits.

\section{Transfer idempotents}

Given a stable $\infty$-category $\mathcal{C}$ and an equivalence $\mathcal{C} \simeq \mathcal{C}^{\prime} \times \mathcal{C}^{\prime \prime}$, every object $X \in \mathcal{C}$ has an essentially unique decomposition $X \simeq X^{\prime} \oplus X^{\prime \prime}$, such that $X^{\prime} \in \mathcal{C}^{\prime}$ and $X^{\prime \prime} \in \mathcal{C}^{\prime \prime}$. This allows us to define a natural endomorphism $\varepsilon: \operatorname{Id}_{\mathcal{C}} \rightarrow \operatorname{Id}_{\mathcal{C}}$ by the formula

$$
X^{\prime} \oplus X^{\prime \prime} \stackrel{\operatorname{Id}_{X^{\prime}} \oplus 0_{X^{\prime \prime}}}{\longrightarrow} X^{\prime} \oplus X^{\prime \prime}
$$

The natural endomorphism $\varepsilon$ is idempotent and realizes internally to $\mathcal{C}$ the projection onto the essential image of $\mathcal{C}^{\prime}$ in $\mathcal{C}$. Our next goal is to provide an explicit description of the idempotent $\varepsilon$ for the split recollement $q^{*}: \mathcal{C}^{B} \hookrightarrow \mathcal{C}^{A}$ of Proposition 4.3.1. To help guide the intuition, we begin with a closely related elementary example:

Example 4.3.3. Let $\mathcal{C}=\operatorname{Vec}_{\mathbb{Q}}$ and let $G$ be a finite group with a normal subgroup $N \triangleleft G$. The map $q: B G \rightarrow B(G / N)$ induces a fully faithful embedding

$$
q^{*}: \operatorname{Vec}_{\mathbb{Q}}^{B(G / N)} \rightarrow \operatorname{Vec}_{\mathbb{Q}}^{B G}
$$

which is the "inflation" functor that takes a vector space $V$ with a $G / N$-action to $V$ itself with the $G$-action induced via $G \rightarrow G / N$. The essential image of $q^{*}$ consists of $G$-representations on which the subgroup $N \triangleleft G$ acts trivially. The adjoints $q_{\text {! }}$ and $q_{*}$ can be identified with the vector spaces of $N$-coinvairant and $N$-invariants respectively, equipped with the residual $G / N$-action. Thus, the full subcategory $\left(\operatorname{Vec}_{\mathbb{Q}}^{B(G / N)}\right)^{\perp} \subseteq \operatorname{Vec}_{\mathbb{Q}}^{B G}$ is spanned by the $G$-representations without non-trivial $N$-fixed vectors and we have an equivalence

$$
\operatorname{Vec}_{\mathbb{Q}}^{B G} \simeq \operatorname{Vec}_{\mathbb{Q}}^{B(G / N)} \times\left(\operatorname{Vec}_{\mathbb{Q}}^{B(G / N)}\right)^{\perp}
$$

This equivalence is realized explicitly as follows. Since $\operatorname{Vec}_{\mathbb{Q}}^{B G}$ is semi-simple, we can split each

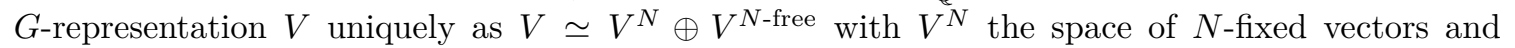
$V^{N \text {-free }} \in\left(\operatorname{Vec}_{\mathbb{Q}}^{B(G / N)}\right)^{\perp}$. Consider the natural endomorphism:

$$
\alpha: \mathrm{Id} \rightarrow(-)_{N} \stackrel{\mathrm{Nm}_{q}}{\longrightarrow}(-)^{N} \rightarrow \mathrm{Id},
$$

and its normalization $\varepsilon:=|N|^{-1} \alpha$. Unwinding the definitions, we get

$$
\varepsilon(x)=\frac{1}{|N|} \sum_{g \in N} g x \quad \in V,
$$

which is an explicit formula for the $G$-equivariant projection $\varepsilon: V \rightarrow V$ onto the subspace $V^{N} \subseteq V$.

In a similar fashion, we have: 
Proposition 4.3.4. Let $\mathcal{C} \in \mathrm{Cat}_{\text {st }}$ and let $A \stackrel{q}{\rightarrow} B$ be a $\mathcal{C}$-acyclic and $\mathcal{C}$-ambidextrous map. Consider

$$
\alpha: \operatorname{Id}_{\mathcal{C}^{A}} \stackrel{u_{!}}{\longrightarrow} q^{*} q_{!} \stackrel{\mathrm{Nm}_{q}}{\longrightarrow} q^{*} q_{*} \stackrel{c_{*}}{\longrightarrow} \operatorname{Id}_{\mathcal{C}^{A}} .
$$

The natural endomorphism $\varepsilon:=q^{*}(|q|) \alpha$ of $\operatorname{Id}_{\mathcal{C}^{A}}$ is idempotent, and it realizes the projection onto the essential image of $q^{*}: \mathcal{C}^{B} \hookrightarrow \mathcal{C}^{A}$.

Proof. First, for every $Y \in\left(\mathcal{C}^{B}\right)^{\perp}$, we have $q_{*} Y=0$ and hence $\left.\alpha\right|_{\left(\mathcal{C}^{B}\right)^{\perp}}=0$. Next, consider the commutative diagram (see Definition 2.1.5):

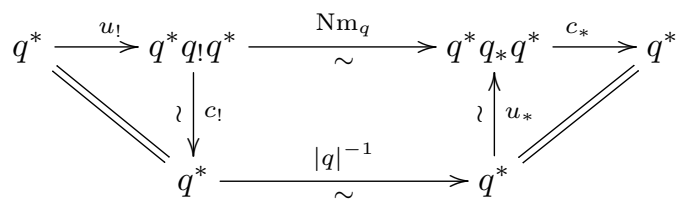

The composition along the top row computes the restriction of $\alpha$ along $q^{*}$, which is therefore invertible and coincides with $q^{*}\left(|q|^{-1}\right)$. It follows that $q^{*}(|q|) \alpha$ is the identity on the essential image of $\mathcal{C}^{B}$ and is zero on $\left(\mathcal{C}^{B}\right)^{\perp}$. Thus, $q^{*}(|q|) \alpha$ equals the idempotent $\varepsilon$ which projects onto the essential image of $\mathcal{C}^{A}$.

Remark 4.3.5. We note that if we furthermore assume that $A \stackrel{q}{\rightarrow} B$ is principal and $B$ is connected, then by Proposition 2.3.3, we have

$$
q^{*}|q|=q^{*} B^{*}|F|=A^{*}|F| .
$$

Namely, $q^{*}|q|$ is constant with value $|F|$. Moreover, by Corollary 2.4.8 and Proposition 2.4.2, the space $\Omega F$ is $\mathcal{C}$-amenable and thus we get $|F|=|\Omega F|^{-1}$. To conclude, we can also write $\varepsilon=|\Omega F|^{-1} \alpha$.

Our main motivation for Proposition 4.3.4, is to generalize Example 4.3.3 to higher heights:

Example 4.3.6. Let $\mathcal{C} \in \mathrm{Cat}_{\mathrm{st}}^{\oplus-\infty}$ be of height $n$. For an abelian $p$-group $G$ with a subgroup $N \leq G$, we consider the fiber sequence

$$
B^{n+1} N \rightarrow B^{n+1} G \stackrel{q}{\rightarrow} B^{n+1}(G / N) .
$$

The map $q$ satisfies the conditions of Theorem 4.3.2, and hence $\mathcal{C}^{B^{n+1} G}$ splits as a product of $\mathcal{C}^{B^{n+1}(G / N)}$ and its right orthogonal complement. Moreover, by Proposition 4.3.4 and Remark 4.3.5, the idempotent $\varepsilon$ is given by $\varepsilon=\left|B^{n} N\right|^{-1} \alpha$. The case $n=0$ is a "derived version" of Example 4.3.3.

We note that Example 4.3.6 is essentially the only interesting case of Theorem 4.3.2, as in view of Proposition 3.2.3(3), for any $n$-connected space $A$, we have $\mathcal{C}^{A} \simeq \mathcal{C}^{B^{n+1} \pi_{n+1} A}$.

\subsection{Chromatic Examples}

The main source of stable higher semiadditive $\infty$-categories is chromatic homotopy theory. In this subsection, we shall address the semiadditive height of such $\infty$-categories, using the properties of nil-conservative functors and the Nilpotence Theorem (see [HS98]). We begin by recalling the following notion:

Definition 4.4.1 ([CSY18, Definition 4.4.1]). We call a functor $F: \mathcal{C} \rightarrow \mathcal{D}$ in $\operatorname{Alg}\left(\operatorname{Pr}_{\mathrm{st}}\right)$ nilconservative, if for every ring $R \in \operatorname{Alg}(\mathcal{C})$, if $F(R)=0$ then $R=0^{11}$.

\footnotetext{
${ }^{11}$ This notion is closely related to the notion of "nil-faithfulness" defined in [Bal16].
} 
We also recall that nil-conservative functors are conservative on the full subcategory of dualizable objects. In particular, we have the following consequence regarding height:

Proposition 4.4.2. Let $F: \mathcal{C} \rightarrow \mathcal{D}$ be a map in $\operatorname{Alg}\left(\operatorname{Pr}_{\mathrm{st}}\right)$. If $\mathcal{C}$ is $m$-semiadditive, then so is $\mathcal{D}$. Furthermore, for every $0 \leq n \leq m$, if $\mathcal{C}$ is of height $\leq n$ or $>n-1$ then so is $\mathcal{D}$ and the converse holds if $F$ is nil-conservative.

Proof. Since $\mathcal{C}$ is $m$-semiadditive and $F: \mathcal{C} \rightarrow \mathcal{D}$ is a monoidal $m$-semiadditive functor, by [CSY18, Corollary 3.3.2(2)], $\mathcal{D}$ is $m$-semiadditive as well. By Corollary 4.2.5, the height of $\mathcal{C}$ (resp. $\mathcal{D}$ ) is determined by the height of $\mathbb{1}_{\mathcal{C}}$ (resp. $\mathbb{1}_{\mathcal{D}}$ ). Moreover, ht $(\mathbb{1}) \leq n$ if and only if $p_{(n)} \in \pi_{0} \mathbb{1}$ is invertible and $\mathrm{ht}(\mathbb{1})>n-1$, if and only if $\left(1-p_{(n-1)} p_{(n)}\right) \in \pi_{0} \mathbb{1}$ is invertible. Thus, the claim follows from [CSY18, Corollary 4.4.5].

As a special case, we get:

Corollary 4.4.3. Let $\mathcal{C} \in \mathrm{CA} \lg \left(\mathrm{Pr}_{\mathrm{st}}\right)$ be $m$-semiadditive and let $R \in \mathrm{CAlg}(\mathcal{C})$. For every integer $0 \leq n \leq m$, if $\mathcal{C}$ is of height $\leq n$ or $>n-1$ then so is $\operatorname{Mod}_{R}(\mathcal{C})$. The converse holds if tensoring with $R$ is nil-conservative.

Proof. The claim follows from Proposition 4.4.2 for the colimit preserving symmetric monoidal functor $R \otimes(-): \mathcal{C} \rightarrow \operatorname{Mod}_{R}(\mathcal{C})$.

We now apply the above to higher semiadditive $\infty$-categories arising in chromatic homotopy theory. We begin with a special case in which cardinalities of Eilenberg-MacLane spaces can be computed explicitly.

Proposition 4.4.4. The $\infty$-category $\Theta_{n}=\operatorname{Mod}_{E_{n}}\left(\operatorname{Sp}_{K(n)}\right)$ satisfies $\operatorname{Ht}\left(\Theta_{n}\right)=n$.

Proof. For $n=0$, the claim is clear, so assume $n \geq 1$. This follows from the explicit formula $p_{(k)}=p^{\left(\begin{array}{c}n-1 \\ k\end{array}\right)}$ given in Proposition 2.2.5. Indeed, for $k \geq n$, we have $p_{(k)}=1$ and hence invertible. For $0 \leq k<n$, the element $p_{(k)}$ is a non-zero power of $p$, and hence every $X \in \Theta_{n}$ is complete with respect to it as the $\infty$-category $\Theta_{n}$ is $p$-complete.

In [CSY18, Theorem $\mathrm{C}]$ we have shown that for a homotopy ring spectrum $R$, the $\infty$-category $\operatorname{Sp}_{R}$ is 1 -semiadditive if and only if it is $\infty$-semiadditive if and only if $\operatorname{supp}(R)=\{n\}$ for some integer $n$. We now show that this $n$ is in fact the semiadditive height of $\operatorname{Sp}_{R}$.

Theorem 4.4.5. Let $R$ be a homotopy ring spectrum ${ }^{12}$. If $\operatorname{supp}(R)=\{n\}$ for some integer $n$, then $\mathrm{Ht}\left(\mathrm{Sp}_{R}\right)=n$. In particular, we have

$$
\operatorname{Ht}\left(\operatorname{Sp}_{K(n)}\right)=\operatorname{Ht}\left(\operatorname{Sp}_{T(n)}\right)=n \text {. }
$$

Proof. We first consider the case $R=K(n)$. This follows by applying Corollary 4.4.3 to the faithful commutative algebra $E_{n} \in \mathrm{CAlg}\left(\mathrm{Sp}_{K(n)}\right)$ and the fact that $\mathrm{Ht}\left(\Theta_{n}\right)=n$ (Proposition 4.4.4). For a general homotopy ring $R$ with $\operatorname{supp}(R)=\{n\}$ (such as $R=T(n)$ ), consider the functor $L_{K(n)}: \operatorname{Sp}_{R} \rightarrow \operatorname{Sp}_{K(n)}$. It is nil-conservative by [CSY18, Proposition 5.1.15]. Since $\operatorname{Sp}_{K(n)}$ is of height $n$, the claim follows from Proposition 4.4.2.

\footnotetext{
${ }^{12}$ In fact, it suffices to assume that $R$ is a weak ring in the sense of [CSY18, Definition 5.1.4].
} 


\section{Modes}

In this section, we use the theory of idempotent algebras in $\mathrm{Pr}$, which we call modes, to further study the interaction of stability and higher semiadditivity.

\section{$5.1 \quad$ Idempotent Algebras}

We begin with a general discussion about idempotent algebras in symmetric monoidal $\infty$-categories, as a means to encode properties, which induce "canonical structure".

\section{Definitions \& characterizations}

Following [Lura, Definition 4.8.2.1], given a symmetric monoidal $\infty$-category $\mathcal{C}$, we say that a morphism $\mathbb{1} \stackrel{u}{\rightarrow} X$ in $\mathcal{C}$ exhibits $X$ as an idempotent object of $\mathcal{C}$, if

$$
X \simeq X \otimes \mathbb{1} \stackrel{1 \otimes u}{\longrightarrow} X \otimes X
$$

is an isomorphism. By [Lura, Proposition 4.8.2.9], an idempotent object $\mathbb{1} \stackrel{u}{\rightarrow} X$ admits a unique commutative algebra structure for which $u$ is the unit. Conversely, the unit $\mathbb{1} \stackrel{u}{\rightarrow} R$ of a commutative algebra $R$, exhibits it as an idempotent object if and only if the multiplication map $R \otimes R \stackrel{m}{\rightarrow} R$ is an isomorphism. In this case we call $R$ an idempotent algebra. More precisely, the functor $\mathrm{CAlg}(\mathcal{C}) \rightarrow \mathcal{C}_{\mathbb{1} /}$ which forgets the algebra structure and remembers only the unit map, induces an equivalence of $\infty$-categories from the full subcategory of idempotent algebras $\operatorname{CAlg}^{\text {idem }}(\mathcal{C}) \subseteq$ $\mathrm{CA} \lg (\mathcal{C})$ to the full subcategory of idempotent objects [Lura, Proposition 4.8.2.9]. The fundamental feature of an idempotent algebra $R$, is that the forgetful functor $\operatorname{Mod}_{R}(\mathcal{C}) \rightarrow \mathcal{C}$ is fully faithful, and its essential image consists of those objects for which the map $Y \otimes \mathbb{1} \stackrel{1 \otimes u}{\longrightarrow} Y \otimes R$ is an isomorphism [Lura, Proposition 4.8.2.10]. Thus, it is a property of an object in $\mathcal{C}$ to have the structure of an $R$-module. We shall say that $R$ classifies the property of being an $R$-module.

Example 5.1.1. For $\mathcal{C}=\mathrm{Ab}$, the idempotent algebras are classically known as solid rings [BK72, Definition 2.1]. These include for example $\mathbb{Q}$ and $\mathbb{F}_{p}$. We note that for $\mathcal{C}=\mathrm{Sp}$, the ring $\mathbb{Q}$ is still idempotent, classifying the property of being rational, but $\mathbb{F}_{p}$ is not idempotent. The idempotent rings in Sp correspond precisely to the smashing localizations.

Given an idempotent ring $R \in \operatorname{CAlg}(\mathcal{C})$, the forgetful functor $\operatorname{Mod}_{R}(\mathcal{C}) \rightarrow \mathcal{C}$ admits a left adjoint

$$
R \otimes(-): \mathcal{C} \rightarrow \operatorname{Mod}_{R}(\mathcal{C}) .
$$

This is a localization functor, which can be thought of as forcing the property classified by $R$ in a universal way. In line with the standard terminology for localizations of spectra, we set:

Definition 5.1.2. Let $L: \mathcal{C} \rightarrow \mathcal{D}$ be a map in $\mathrm{CAlg}\left(\mathrm{Cat}_{\infty}\right)$. We say that $L$ is a smashing localization if there exists an idempotent algebra $R$ in $\mathcal{C}$ and an isomorphism $\operatorname{Mod}_{R}(\mathcal{C}) \stackrel{\sim}{\longrightarrow} \mathcal{D}$ in $\mathrm{CAlg}\left(\mathrm{Cat}_{\infty}\right)$, such that $L$ is the composition

$$
\mathcal{C} \stackrel{R \otimes(-)}{\longrightarrow} \operatorname{Mod}_{R}(\mathcal{C}) \stackrel{\sim}{\longrightarrow} \mathcal{D} .
$$


We note that for a smashing localization $L: \mathcal{C} \rightarrow \mathcal{D}$, there is always a fully faithful right adjoint $F: \mathcal{D} \rightarrow \mathcal{C}$, which is lax symmetric monoidal [Lura, Corollary 7.3.2.7], and we can identify the idempotent algebra $R$ with $F L\left(\mathbb{1}_{\mathcal{C}}\right)$. To characterize smashing localizations, we first introduce some terminology. Let $L: \mathcal{C} \rightarrow \mathcal{D}$ in $\operatorname{CAlg}\left(\mathrm{Cat}_{\infty}\right)$, which admits a (lax symmetric monoidal) right adjoint $F: \mathcal{D} \rightarrow \mathcal{C}$. For every $X \in \mathcal{C}$ and $Y \in \mathcal{D}$, we have a natural map

$$
X \otimes F(Y) \stackrel{\alpha}{\rightarrow} F(L(X) \otimes Y)
$$

which is the mate of

$$
L(X \otimes F(Y)) \simeq L(X) \otimes L F(Y) \stackrel{L(X) \otimes c_{Y}}{\longrightarrow} L(X) \otimes Y
$$

where $c: L F \rightarrow \mathrm{Id}$ is the counit of the adjunction. We say that the adjunction $L \dashv F$ satisfies the projection formula if the map $\alpha$ is an isomorphism for all $X, Y$. The map $\alpha$ is compatible with the unit of the adjunction $\mathrm{Id} \stackrel{u}{\rightarrow} F L$ in the following sense:

Lemma 5.1.3. For all $X, Z \in \mathcal{C}$, the following diagram is commutative

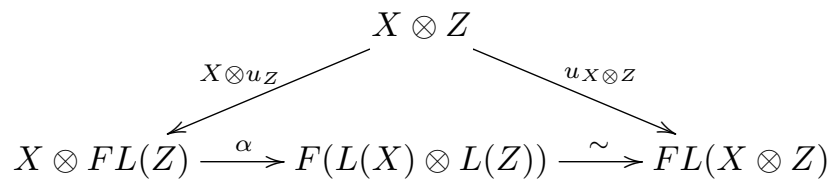

Proof. Passing to the mates under the adjunction $L \dashv F$ and unwinding the definitions, this follows from the zigzag identities.

We can now characterize the smashing localizations among all localizations as those that satisfy the projection formula (compare with [MNN17, Proposition 5.29]):

Proposition 5.1.4. A map $L: \mathcal{C} \rightarrow \mathcal{D}$ in $\mathrm{CAlg}\left(\mathrm{Cat}_{\infty}\right)$ is a smashing localization if and only if it admits a fully faithful right adjoint $F: \mathcal{D} \rightarrow \mathcal{C}$ (i.e. it is a localization) and the adjunction $L \dashv F$ satisfies the projection formula.

Proof. In the "only if" direction, the right adjoint of $R \otimes(-): \mathcal{C} \rightarrow \operatorname{Mod}_{R}(\mathcal{C})$ is the forgetful functor $F: \operatorname{Mod}_{R}(\mathcal{C}) \rightarrow \mathcal{C}$, which is fully faithful since $R$ is idempotent. For $X \in \mathcal{C}$ and $M \in \operatorname{Mod}_{R}(\mathcal{C})$, the projection formula transformation is the composition

$$
X \otimes F(M) \stackrel{u_{(X \otimes F(M))}}{\longrightarrow} F L(X \otimes F(M)) \stackrel{\sim}{\longrightarrow} F(L(X) \otimes L F(M)) \stackrel{L(X) \otimes c_{M}}{\longrightarrow} F(L(X) \otimes M) .
$$

The map $c_{M}$ is an isomorphism since $F$ is fully faithful. The map $u_{(X \otimes F(M))}$ is an isomorphism because $X \otimes F(M)$ admits a structure of an $R$-module and hence in the essential image of $L$.

For the "if" direction, consider the commutative ring

$$
R=F L\left(\mathbb{1}_{\mathcal{C}}\right)=F\left(\mathbb{1}_{\mathcal{D}}\right) .
$$

By the projection formula, we have a natural isomorphism

$$
X \otimes R \simeq X \otimes F\left(\mathbb{1}_{\mathcal{D}}\right) \simeq F\left(L(X) \otimes \mathbb{1}_{\mathcal{D}}\right) \simeq F L(X) .
$$


Since $F$ is fully faithful, the unit map Id $\stackrel{u}{\rightarrow} F L$ exhibits $F L: \mathcal{C} \rightarrow \mathcal{C}$ as a localization (as in [Lur09, Proposition 5.2.7.4]). Moreover, by Lemma 5.1.3, the unit map Id $\stackrel{u}{\rightarrow} F L$ is induced from tensoring with the unit $\mathbb{1}_{\mathcal{C}} \stackrel{u}{\rightarrow} R$ of $R$. Thus, by [Lura, Proposition 4.8.2.4], $R$ is an idempotent ring. Moreover, by [Lura, Proposition 4.8.2.10], the forgetful functor $\operatorname{Mod}_{R}(\mathcal{C}) \rightarrow \mathcal{C}$ is a symmetric monoidal equivalence onto the essential image of $F L$ with the localized symmetric monoidal structure, which is equivalent to $\mathcal{D}$.

Remark 5.1.5. Let $\mathcal{C}$ be presentably symmetric monoidal and let $R$ be an idempotent algebra in $\mathcal{C}$. The fully faithful forgetful functor $F: \operatorname{Mod}_{R}(\mathcal{C}) \hookrightarrow \mathcal{C}$ admits also a right adjoint. Hence, if $\mathcal{C}$ is moreover stable, then $F$ exhibits $\mathcal{C}$ as a recollement of $\operatorname{Mod}_{R}(\mathcal{C})$ and its right orthogonal complement.

\section{Poset structure}

Another nice characterization of idempotent algebras is as the $(-1)$-cotruncated objects of $\operatorname{CAlg}(\mathcal{C})$ :

Proposition 5.1.6. Let $\mathcal{C} \in \mathrm{CA} \lg \left(\mathrm{Cat}_{\infty}\right)$. A commutative algebra $R \in \mathrm{CAlg}(\mathcal{C})$ is idempotent, if and only if for all $S \in \mathrm{CAlg}(\mathcal{C})$ the space $\operatorname{Map}_{\mathrm{CAlg}(\mathcal{C})}(R, S)$ is either empty or contractible. Moreover, it is non-empty if and only if $S$ is an $R$-module.

Proof. By [Lura, Proposition 3.2.4.7], the tensor product of commutative algebras is the coproduct in $\mathrm{CAlg}(\mathcal{C})$. Moreover, the multiplication map

$$
R \sqcup R=R \otimes R \stackrel{m}{\longrightarrow} R
$$

is the categorical fold map. Thus, it follows by the dual of [Lur09, Lemma .5.5.6.15], that $R$ is idempotent if and only if it is $(-1)$-cotruncated. We note that [Lur09, Lemma .5.5.6.15] requires the $\infty$-category to admit finite limits; however, the only limit used in the proof is the one defining the diagonal map, which in our case corresponds to the coproduct defining the fold map. Now, if there exists a map $R \rightarrow S$ in $\operatorname{CAlg}(\mathcal{C})$, then $S$ is an $R$-algebra and in particular an $R$-module. Conversely, for every $S \in \operatorname{CAlg}(\mathcal{C})$, we have maps

$$
\begin{aligned}
& R=R \otimes \mathbb{1} \stackrel{1 \otimes u_{S}}{\longrightarrow} R \otimes S \\
& S=\mathbb{1} \otimes S \stackrel{u_{R} \otimes 1}{\longrightarrow} R \otimes S
\end{aligned}
$$

in $\mathrm{CAlg}(\mathcal{C})$. If $S$ is an $R$-module, then the map $u_{R} \otimes 1$ is an isomorphism, and thus

$$
\left(u_{R} \otimes 1\right)^{-1} \circ\left(1 \otimes u_{S}\right): R \rightarrow S
$$

is a map in $\operatorname{CAlg}(\mathcal{C})$.

We also have the following non-commutative analogue of Proposition 5.1.6:

Proposition 5.1.7. Let $\mathcal{C} \in \mathrm{CAlg}\left(\mathrm{Cat}_{\infty}\right)$ and let $R \in \mathrm{CAlg}^{\mathrm{idem}}(\mathcal{C})$. For every algebra $S \in \operatorname{Alg}(\mathcal{C})$, the space $\operatorname{Map}_{\operatorname{Alg}(\mathcal{C})}(R, S)$ is either empty or contractible and it is non-empty if and only if $S$ is an $R$-module. 
Proof. As in the proof of Proposition 5.1.6, $S$ is an $R$-module if and only if there exists a map $R \rightarrow S$ in $\operatorname{Alg}(\mathcal{C})$. If $S$ is an $R$-module, then $S \in \operatorname{Alg}\left(\operatorname{Mod}_{R}(\mathcal{C})\right)$ and

$$
\operatorname{Map}_{\operatorname{Alg}(\mathcal{C})}(R, S) \simeq \operatorname{Map}_{\operatorname{Alg}\left(\operatorname{Mod}_{R}(\mathcal{C})\right)}(R, S) \simeq \mathrm{pt},
$$

since $R$ is the initial object of $\operatorname{Alg}_{R}(\mathcal{C})=\operatorname{Alg}\left(\operatorname{Mod}_{R}(\mathcal{C})\right)$.

As a consequence of Proposition 5.1.6, the $\infty$-category $\operatorname{CAlg}^{\text {idem }}(\mathcal{C})$ of idempotent algebras is a poset. This poset admits binary meets:

Proposition 5.1.8. Let $\mathcal{C} \in \mathrm{CAlg}\left(\mathrm{Cat}_{\infty}\right)$ and $R, S \in \mathrm{CAlg}^{\mathrm{idem}}(\mathcal{C})$. Then $R \otimes S$ is an idempotent algebra which classifies the conjunction of the properties classified by $R$ and $S$.

Proof. Let $\mathbb{1} \stackrel{u_{R}}{\longrightarrow} R$ be the unit of $R$ and $\mathbb{1} \stackrel{u_{S}}{\longrightarrow} S$ the unit of $S$. We consider the composition

$$
u_{R \otimes S}: \mathbb{1} \simeq \mathbb{1} \otimes \mathbb{1} \stackrel{u_{R} \otimes 1}{\longrightarrow} R \otimes \mathbb{1} \stackrel{1 \otimes u_{S}}{\longrightarrow} R \otimes S .
$$

After tensoring with $R$ the first map becomes an isomorphism and after tensoring with $S$ the second map becomes an isomorphism. Thus, $u_{R \otimes S}$ exhibits $R \otimes S$ as an idempotent object. Given an $R \otimes S$-module $M$, tensoring $\mathbb{1} \stackrel{u_{R \otimes S}}{\longrightarrow} R \otimes S$ with $M$ is an isomorphism. Thus, $M \simeq R \otimes S \otimes M$ and hence $M$ is both an $R$-module and an $S$-module. Conversely, tensoring $\mathbb{1} \stackrel{u_{R \otimes S}}{\longrightarrow} R \otimes S$ with $M$ is given by the composition:

$$
\mathbb{1} \otimes \mathbb{1} \otimes M \stackrel{u_{R} \otimes 1 \otimes 1}{\longrightarrow} R \otimes \mathbb{1} \otimes M \stackrel{1 \otimes u_{S} \otimes 1}{\longrightarrow} R \otimes S \otimes M
$$

Hence, if $M$ is both an $R$-module and an $S$-module, then tensoring $\mathbb{1} \stackrel{u_{R}}{\longrightarrow} R$ and $\mathbb{1} \stackrel{u_{S}}{\longrightarrow} S$ with $M$ is an isomorphism, and so tensoring $\mathbb{1} \stackrel{u_{R \otimes \mathcal{S}}}{\longrightarrow} R \otimes S$ with $M$ is an isomorphism as well. Thus, $M$ is an $R \otimes S$-module.

Idempotent objects are also closed under sifted colimits in $\mathcal{C}$.

Proposition 5.1.9. Let $\mathcal{C} \in \mathrm{CAlg}\left(\mathrm{Cat}_{\infty}\right)$, and let $\mathcal{J}$ be a sifted $\infty$-category such that $\mathcal{C}$ is compatible with $\mathcal{J}$-indexed colimits. Then

(1) The $\infty$-category $\mathrm{CAlg}^{\mathrm{idem}}(\mathcal{C})$ admits $\mathcal{J}$-indexed colimits.

(2) The forgetful functor $\mathrm{CAlg}{ }^{\text {idem }}(\mathcal{C}) \rightarrow \mathcal{C}$ preserves $\mathcal{J}$-indexed colimits.

(3) Given a functor $F: \mathcal{J} \rightarrow \mathrm{CAlg}^{\text {idem }}(\mathcal{C})$, the idempotent algebra $\lim _{\longrightarrow} F$ classifies the conjunction of the properties classified by $F(j)$ for all $j \in \mathcal{J}$.

Proof. By [Lura, Corollary 3.2.3.2] the $\infty$-category $\operatorname{CAlg}(\mathcal{C})$ admits $\mathcal{J}$-indexed colimits and the forgetful functor $\operatorname{CAlg}(\mathcal{C}) \rightarrow \mathcal{C}$ preserves $\mathcal{J}$-indexed colimits. Thus, to prove (1) and (2) it suffices to show that $\mathrm{CAlg}{ }^{\text {idem }}(\mathcal{C}) \subseteq \operatorname{CAlg}(\mathcal{C})$ is closed under $\mathcal{J}$-indexed colimits. Since $\mathcal{J}$ is sifted, the diagonal map $\mathcal{J} \rightarrow \mathcal{J} \times \mathcal{J}$ is cofinal. Therefore, given a functor $F: \mathcal{J} \rightarrow \operatorname{CAlg}(\mathcal{C})$, the multiplication map

$$
\stackrel{\lim }{\longrightarrow} F \otimes \lim _{\longrightarrow} F \rightarrow \underline{\lim } F
$$

can be identified with the colimit of the multiplication maps $F(j) \otimes F(j) \rightarrow F(j)$ for $j \in \mathcal{J}$. Hence, if $F(j)$ is an idempotent algebra for every $j \in \mathcal{J}$, so is $\lim _{\longrightarrow} F$. We shall now prove (3). First, for 
every $j \in \mathcal{J}$ there is a canonical algebra map $F(j) \rightarrow \varliminf_{\longrightarrow} F$. Thus, every $\left(\lim _{\longrightarrow} F\right.$ )-module admits an $F(j)$-module structure for every $j \in \mathcal{J}$. It remains to prove that if $M \in \mathcal{C}$ admits an $F(j)$-module structure for every $j \in \mathcal{J}$, then

$$
M \otimes \mathbb{1} \stackrel{1 \otimes u}{\longrightarrow} M \otimes \lim _{\longrightarrow} F
$$

is an isomorphism. Since $\mathcal{C}$ is compatible with $\mathcal{J}$-indexed colimits, the map $1 \otimes u$ above is the colimit of the isomorphisms $M \otimes \mathbb{1} \stackrel{1 \otimes u_{j}}{\longrightarrow} M \otimes F(j)$.

Consequently, under mild conditions on $\mathcal{C}$, the poset $\operatorname{CAlg}^{\text {idem }}(\mathcal{C})$ admits arbitrary meets.

Corollary 5.1.10. Let $\mathcal{C} \in \mathrm{CA} \lg \left(\mathrm{Cat}_{\infty}\right)$ which is compatible with filtered colimits. Then the poset $\mathrm{CAlg}^{\text {idem }}(\mathcal{C})$ is cocomplete. Moreover, given a functor $F: \mathcal{J} \rightarrow \operatorname{CAlg}^{\text {idem }}(\mathcal{C})$, the idempotent algebra

$$
\lim _{\longrightarrow} F=\sup _{j \in \mathcal{J}} F(j)
$$

classifies the conjunction of the properties classified by $F(j)$ for all $j \in \mathcal{J}$.

Proof. First, $\operatorname{CAlg}{ }^{\text {idem }}(\mathcal{C})$ admits an initial object which is $\mathbb{1}_{\mathcal{C}}$. Second, by Proposition 5.1.8, CAlg ${ }^{\text {idem }}$ admits binary coproducts. Since every filtered $\infty$-category is sifted by [Lur09, Example 5.5.8.3] we get by Proposition 5.1.9 that $\mathrm{CAlg}{ }^{\text {idem }}(\mathcal{C})$ admits filtered colimits. Since $\mathrm{CAlg}^{\text {idem }}(\mathcal{C})$ is a poset, we deduce that it is cocomplete. Furthermore, Proposition 5.1.8 and Proposition 5.1.9 also imply that the colimit classifies the conjunction of the properties classified by the idempotent algebras in the diagram.

Under some conditions, disjoint idempotent algebras have also binary joins.

Proposition 5.1.11. Let $\mathcal{C} \in \mathrm{CAlg}\left(\mathrm{Cat}_{\infty}\right)$ which is compatible with all small colimits and is 0semiadditive. Let $R, S$ be idempotent algebras in $\mathcal{C}$. If $R \otimes S \simeq 0$, then $R \times S$ is an idempotent algebra, which classifies the property of an object $X \in \mathcal{C}$ to be of the form $X_{R} \oplus X_{S}$, where $X_{R}$ is an $R$-module and $X_{S}$ is an $S$-module.

Proof. By assumption, the tensor product preserves binary coproducts in each variable. Since $\mathcal{C}$ is 0 -semiadditive, we get that the tensor product also preserves binary products in each variable. Thus,

$$
(R \otimes R) \times(S \otimes S) \simeq(R \times S) \otimes(R \times S) \stackrel{m_{R \times S}}{\longrightarrow} R \times S
$$

coincides with $m_{R} \times m_{S}$, which is an isomorphism. Thus, $m_{R \times S}$ is an isomorphism, and therefore, $R \times S$ is an idempotent algebra. The projection maps $R \times S \rightarrow R$ and $R \times S \rightarrow S$ induce the extension of scalars functors

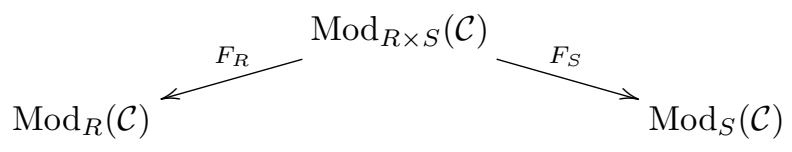

which in turn induce a functor

$$
F: \operatorname{Mod}_{R \times S}(\mathcal{C}) \rightarrow \operatorname{Mod}_{R}(\mathcal{C}) \times \operatorname{Mod}_{S}(\mathcal{C}) .
$$


Since $F_{R}$ and $F_{S}$ are left adjoints, by [HY17, Theorem B] the functor $F$ admits a right adjoint $G$, which is given object-wise by $G\left(X_{R}, X_{S}\right) \simeq X_{R} \times X_{S}$. To complete the proof of the claim, it would suffice to show that $G$ is an equivalence. We do this by showing that $G$ is conservative and $F$ is fully faithful. By Lemma 3.3.1, in order to show that $F$ is fully faithful, it suffices to show that $G F$ is an equivalence. For every $(R \times S)$-module $X$, we have

$$
G F(X)=\left(R \otimes_{R \times S} X\right) \times\left(S \otimes_{R \times S} X\right) \simeq(R \times S) \otimes_{R \times S} X \simeq X .
$$

To show that $G$ is conservative, it suffices to observe that the underlying $\mathcal{C}$-object of $G\left(X_{R}, X_{S}\right)$ is the direct sum $X_{R} \oplus X_{S}$ and both $X_{R}$ and $X_{S}$ are retracts of $X_{R} \oplus X_{S}$.

\section{$5.2 \quad$ Theory of Modes}

We now specialize the notion of idempotent algebras to the $\infty$-category $\operatorname{Pr}$ of presentable $\infty$ categories and colimit preserving functors.

\section{Tensor of presentable $\infty$-categories}

Recall from [Lura, Proposition 4.8.1.15], that the $\infty$-category Pr admits a closed symmetric monoidal structure. The unit is $\mathcal{S} \in \operatorname{Pr}$, and for every $\mathcal{C}, \mathcal{D} \in \operatorname{Pr}$, the internal hom and tensor product are given respectively by

$$
\operatorname{hom}(\mathcal{C}, \mathcal{D})=\operatorname{Fun}^{L}(\mathcal{C}, \mathcal{D}), \quad \mathcal{C} \otimes \mathcal{D}=\operatorname{Fun}^{R}\left(\mathcal{C}^{o p}, \mathcal{D}\right) \simeq \operatorname{Fun}^{R}\left(\mathcal{D}^{o p}, \mathcal{C}\right) .
$$

It is worth spelling out in what sense the above formula for the tensor product is functorial. Given a functor $\mathcal{D}_{1} \stackrel{F}{\rightarrow} \mathcal{D}_{2}$ in Pr with right adjoint $G$, the induced functor $\mathcal{C} \otimes \mathcal{D}_{1} \stackrel{\operatorname{Id}_{\mathcal{C}} \otimes F}{\longrightarrow} \mathcal{C} \otimes \mathcal{D}_{2}$ is the left adjoint of

$$
\operatorname{Fun}^{R}\left(\mathcal{C}^{o p}, \mathcal{D}_{2}\right) \stackrel{G \circ(-)}{\longrightarrow} \operatorname{Fun}^{R}\left(\mathcal{C}^{o p}, \mathcal{D}_{1}\right) .
$$

From this we get that tensoring with $\mathcal{C}$ preserves reflective localizations:

Lemma 5.2.1. Let $\mathcal{C}$ and $\mathcal{D}_{1} \stackrel{F}{\rightarrow} \mathcal{D}_{2}$ in Pr. If $F$ admits a fully faithful (resp. conservative) right adjoint, then so does $\operatorname{Id}_{\mathcal{C}} \otimes F$.

Proof. By the above formula for $\operatorname{Id}_{\mathcal{C}} \otimes F$, its right adjoint is given by

$$
\operatorname{Fun}^{R}\left(\mathcal{C}^{o p}, \mathcal{D}_{2}\right) \stackrel{G \circ(-)}{\longrightarrow} \operatorname{Fun}^{R}\left(\mathcal{C}^{o p}, \mathcal{D}_{1}\right),
$$

where $G$ is the right adjoint of $F$. Thus, if $G$ is fully faithful (resp. conservative), then post composition with $G$ is fully faithful (resp. conservative) as well.

Remark 5.2.2. On the other hand, if $\mathcal{D}_{1} \stackrel{F}{\rightarrow} \mathcal{D}_{2}$ is itself fully faithful or conservative, then $\operatorname{Id}_{\mathcal{C}} \otimes F$ need not be. For example, let $\mathrm{Sp}^{\mathrm{cn}} \subseteq \mathrm{Sp}$ be the full subcategory of connective spectra. One can show that $\mathrm{Sp}^{\mathrm{cn}} \otimes \mathrm{Set} \simeq \mathrm{Ab}$, while $\mathrm{Sp} \otimes \mathrm{Set} \simeq 0$ (e.g. by Proposition 5.2.10). Thus, tensoring the fully faithful inclusion $\mathrm{Sp}^{\mathrm{cn}} \hookrightarrow \mathrm{Sp}$ with the category Set, produces the zero functor $\mathrm{Ab} \rightarrow 0$.

Another general fact which we shall require is the preservation of recollements under base change. Recall that $\operatorname{Pr}_{\text {st }} \subseteq \operatorname{Pr}$ is the full subcategory of stable presentable $\infty$-categories. 
Proposition 5.2.3. Let $\mathcal{C} \in \operatorname{Pr}_{\text {st }}$ and assume it is a recollement of $\mathcal{C}_{\circ} \subseteq \mathcal{C}$ and $\mathcal{C}_{\circ}^{\perp} \subseteq \mathcal{C}$. For every $\mathcal{D} \in \operatorname{Pr}$, the morphism $\mathcal{C}_{\circ} \otimes \mathcal{D} \rightarrow \mathcal{C} \otimes \mathcal{D}$ exhibits $\mathcal{C} \otimes \mathcal{D}$ as a recollement of $\mathcal{C}_{\circ} \otimes \mathcal{D}$ and $\left(\mathcal{C}_{\circ} \otimes \mathcal{D}\right)^{\perp} \simeq \mathcal{C}_{\circ}^{\perp} \otimes \mathcal{D}$.

Proof. Let $\mathcal{C}_{\circ} \stackrel{F}{\rightarrow} \mathcal{C}$ be the inclusion functor. We denote by $\mathcal{C} \stackrel{L}{\rightarrow} \mathcal{C}_{\circ}$ and $\mathcal{C} \stackrel{R}{\rightarrow} \mathcal{C}_{\circ}$ the left and right adjoints of $F$ respectively. We observe that $\mathcal{C}_{\circ}$ is presentable as an accessible localization of $\mathcal{C}$, and hence both functors $F$ and $L$ are morphisms in Pr. The adjunction $F \dashv R$ induces an adjunction

$$
F \circ(-): \operatorname{Fun}\left(\mathcal{D}^{o p}, \mathcal{C}_{\circ}\right) \leftrightarrows \operatorname{Fun}\left(\mathcal{D}^{o p}, \mathcal{C}\right): R \circ(-)
$$

Since $F$ and $R$ are both right adjoints, this adjunction restricts to an adjunction on the full subcategories spanned by the right adjoints on both sides,

$$
F \circ(-): \mathcal{D} \otimes \mathcal{C}_{\circ} \simeq \operatorname{Fun}^{R}\left(\mathcal{D}^{o p}, \mathcal{C}_{\circ}\right) \leftrightarrows \operatorname{Fun}^{R}\left(\mathcal{D}^{o p}, \mathcal{C}\right) \simeq \mathcal{D} \otimes \mathcal{C}: R \circ(-)
$$

On the other hand, the left adjoint of $R \circ(-)$ is $F \otimes \mathcal{D}$ and the left adjoint of $F \circ(-)$ is $L \otimes \mathcal{D}$. It follows that $L \otimes \mathcal{D}$ is the left adjoint of $F \otimes \mathcal{D}$. To conclude, $F \otimes \mathcal{D}$ admits a right adjoint (as a morphism in Pr) and also a left adjoint, given by $L \otimes \mathcal{D}$. Furthermore, by Lemma 5.2.1, $F \otimes \mathcal{D}$ is also fully faithful. The $\infty$-categories $\mathcal{C}_{\circ} \otimes \mathcal{D}$ and $\mathcal{C} \otimes \mathcal{D}$ are stable by [Lura, Proposition 4.8.2.18] because $\mathcal{C}_{\circ}$ and $\mathcal{C}$ are. Hence, we deduce that $F \otimes \mathcal{D}$ exhibits $\mathcal{C} \otimes \mathcal{D}$ as a recollement of $\mathcal{C}_{\circ} \otimes \mathcal{D}$ and $\left(\mathcal{C}_{\circ} \otimes \mathcal{D}\right)^{\perp}$.

It remains to identify $\left(\mathcal{C}_{\circ} \otimes \mathcal{D}\right)^{\perp}$ with $\mathcal{C}_{\circ}^{\perp} \otimes \mathcal{D}$. Note that $\left(\mathcal{C}_{\circ} \otimes \mathcal{D}\right)^{\perp}$ is the full subcategory of $\mathcal{C} \otimes \mathcal{D}$ spanned by objects on which the right adjoint of $F \otimes \mathcal{D}$ is zero. This right adjoint is given by

$$
R \circ(-): \operatorname{Fun}^{R}\left(\mathcal{D}^{o p}, \mathcal{C}\right) \rightarrow \operatorname{Fun}^{R}\left(\mathcal{D}^{o p}, \mathcal{C}_{\circ}\right) .
$$

On the other hand, the inclusion $F^{\perp}: \mathcal{C}_{\circ}^{\perp} \hookrightarrow \mathcal{C}$ is right adjoint to its left adjoint $L^{\perp}: \mathcal{C} \rightarrow \mathcal{C}_{\circ}^{\perp}$, and hence the right adjoint of $L^{\perp} \otimes \mathcal{D}$ is given by

$$
F^{\perp} \circ(-): \operatorname{Fun}^{R}\left(\mathcal{D}^{o p}, \mathcal{C}_{\circ}^{\perp}\right) \rightarrow \operatorname{Fun}^{R}\left(\mathcal{D}^{o p}, \mathcal{C}\right) .
$$

This is a fully faithful functor whose essential image consists precisely of objects on which $R \circ(-)$ is zero. Thus, we have canonically identified $\left(\mathcal{C}_{\circ} \otimes \mathcal{D}\right)^{\perp}$ with $\mathcal{C}_{\circ}^{\perp} \otimes \mathcal{D}$.

\section{Definition \& examples of modes}

We are now ready to introduce the central notion of this section:

Definition 5.2.4. A mode is an idempotent algebra in Pr. We denote by

$$
\text { Mode }:=\mathrm{CAlg}^{\text {idem }}(\mathrm{Pr}) \subseteq \mathrm{CAlg}(\mathrm{Pr})
$$

the full subcategory spanned by modes.

Applying the preceding results on idempotent algebras to Pr, we get the following:

\section{Proposition 5.2.5.}

(1) Mode is a (large) poset. 
(2) Mode is co-complete. Moreover, the colimit of a diagram of modes classifies the conjunction of the properties classified by the modes in the diagram.

(3) $\mathcal{S} \in$ Mode is the initial mode and it classifies the empty property (which is always satisfied).

(4) $\mathcal{M} \sqcup \mathcal{N}=\mathcal{M} \otimes \mathcal{N}$ for all $\mathcal{M}, \mathcal{N} \in$ Mode.

(5) The forgetful functor Mode $\rightarrow \operatorname{Pr}$ preserves sifted colimits.

(6) $0 \in$ Mode $i s$ the terminal mode and it classifies the property of being equivalent to 0.

(7) For $\mathcal{M}, \mathcal{N} \in$ Mode, if $\mathcal{M} \otimes \mathcal{N}=0$, then their join is given by $\mathcal{M} \times \mathcal{N}$, and it classifies the property of being a direct product of an $\mathcal{M}$-module and an $\mathcal{N}$-module.

Proof. (1) follows from Proposition 5.1.6. (2) follows from Corollary 5.1.10 and the fact that $\operatorname{Pr}$ is closed symmetric monoidal. (3) follows from the fact that $\mathcal{S}$ is the unit of Pr. (4) follows from Proposition 5.1.8. (5) follows from Proposition 5.1.9. (6) follows from the fact that 0 is a zero object of Pr. (7) follows from Proposition 5.1.11, since Pr is 0-semiadditive ([HL13, Example 4.3.11]).

In addition to the initial and terminal modes, we also have the following (far from exhaustive) list of modes: ${ }^{13}$

\section{Example 5.2.6.}

(1) $(0 \rightarrow 1)$ is the boolean mode which classifies the property of being equivalent to a poset (i.e. the mode of propositional logic).

(2) Set is the discrete mode, which classifies the property of being equivalent to an ordinary category (i.e. the mode of ordinary, as opposed to "higher", mathematics).

(3) $\mathrm{Ab}$ is the discrete additive mode, which classifies the property of being equivalent to an ordinary additive category.

(4) $\mathcal{S}_{*}$ is the pointed mode, which classifies the property of having a zero object.

(5) Sp is the stable mode, which classifies the property of being stable.

Given a mode $\mathcal{M}$, the fully faithful forgetful functor $\operatorname{Mod}_{\mathcal{M}}(\operatorname{Pr}) \hookrightarrow \operatorname{Pr}$ admits a left adjoint (i.e. localization), which is given by

$$
\mathcal{C} \mapsto \mathcal{M} \otimes \mathcal{C}=\operatorname{Fun}^{R}\left(\mathcal{M}^{o p}, \mathcal{C}\right) .
$$

This procedure should be thought of as forcing $\mathcal{C}$ to be in the mode $\mathcal{M}$ in a universal way.

Example 5.2.7. For the stable mode $\mathrm{Sp}$, the $\infty$-category $\operatorname{Sp} \otimes \mathcal{C}$ is the stabilization $\operatorname{Sp}(\mathcal{C}) \in \operatorname{Pr}_{\mathrm{st}}$ [Lura, Example 4.8.1.23]. Similarly, for the discrete mode Set, the $\infty$-category Set $\otimes \mathcal{C}$ is the 0truncation $\tau_{\leq 0} \mathcal{C}$ [Lura, Remark 4.8.2.17].

The general results for idempotent algebras have the following implication:

\footnotetext{
${ }^{13}$ All these can be found in [Lura, Section 4.8.2] with the exception of (3), which can be deduced from Proposition 5.2.10.
} 
Proposition 5.2.8. Let $\mathcal{M}$ be a mode and $\mathcal{C} \in \operatorname{Alg}(\operatorname{Pr})$ which is an $\mathcal{M}$-module. The fully faithful embedding $\operatorname{Mod}_{\mathcal{M}}(\operatorname{Pr}) \hookrightarrow \operatorname{Pr}$ induces an equivalence of $\infty$-categories

$$
\operatorname{LMod}_{\mathcal{C}}\left(\operatorname{Mod}_{\mathcal{M}}(\operatorname{Pr})\right) \stackrel{\sim}{\longrightarrow} \operatorname{LMod}_{\mathcal{C}}(\operatorname{Pr}) .
$$

In particular, every $\mathcal{D} \in \operatorname{LMod}_{\mathcal{C}}(\operatorname{Pr})$ is an $\mathcal{M}$-module.

Proof. Since $\mathcal{C}$ is an $\mathcal{M}$-module, by Proposition 5.1.7, there is a map of algebras $\mathcal{M} \rightarrow \mathcal{C}$ and the claim follows.

The $(\infty, 2)$-categorical structure of Pr allows further constructions of modes beyond those provided by Proposition 5.2.5. Primarily, modes can be localized.

\section{Localization of modes}

Given a mode $\mathcal{M}$, every $\mathcal{M}$-module $\mathcal{C}$ is by definition left-tensored over $\mathcal{M}$, and hence in particular enriched over $\mathcal{M}$ [Lura, Proposition 4.2.1.33]. For every $X, Y \in \mathcal{C}$, we denote by $\operatorname{hom}^{\mathcal{M}}(X, Y)$ the corresponding hom-object in $\mathcal{M}$. The $\mathcal{M}$-enrichment of an $\mathcal{M}$-module $\mathcal{C}$ can be explicitly described via the $\mathcal{M}$-enriched Yoneda embedding:

$$
\mathcal{C} \simeq \mathcal{C} \otimes \mathcal{M} \simeq \operatorname{Fun}^{R}\left(\mathcal{C}^{o p}, \mathcal{M}\right) \hookrightarrow \operatorname{Fun}\left(\mathcal{C}^{o p}, \mathcal{M}\right) .
$$

Definition 5.2.9. Let $\mathcal{M}$ be a mode, let $\mathcal{M} \circ \subseteq \mathcal{M}$ be a reflective full subcategory, and let $\mathcal{C}$ be an $\mathcal{M}$-module. We say that an object $X \in \mathcal{C}$ is $\mathcal{M}_{\circ}$-local if for every $Z \in \mathcal{C}$, the enriched hom-object $\operatorname{hom}^{\mathcal{M}}(Z, X)$ lies in $\mathcal{M}_{\circ}$. Furthermore, we say that $\mathcal{C}$ itself is $\mathcal{M}_{\circ}$-local, if every object of $\mathcal{C}$ is $\mathcal{M}_{\circ}$-local.

Proposition 5.2.10. Let $\mathcal{M}$ be a mode and $\mathcal{M} \circ \subseteq \mathcal{M}$ an accessible reflective full subcategory, which is compatible with the symmetric monoidal structure of $\mathcal{C}$. Let $L$ be the left adjoint of the inclusion $\mathcal{M}_{\circ} \hookrightarrow \mathcal{M}$. The composition

$$
\mathcal{S} \stackrel{u}{\rightarrow} \mathcal{M} \stackrel{L}{\rightarrow} \mathcal{M} \text { 。 }
$$

exhibits $\mathcal{M}_{\circ}$ as a mode. Moreover, for every $\mathcal{M}$-module $\mathcal{C}$, the $\infty$-category $\mathcal{M}_{\circ} \otimes \mathcal{C}$ can be canonically identified with the full subcategory of $\mathcal{M}_{\circ}$-local objects in $\mathcal{C}$. In particular, $\mathcal{M}_{\circ}$ classifies the property of being an $\mathcal{M}_{\circ}$-local $\mathcal{M}$-module.

Proof. By [Lura, Proposition 2.2.1.9], the $\infty$-category $\mathcal{M}_{\circ}$ admits a canonical symmetric monoidal structure, such that the left adjoint $\mathcal{M} \stackrel{L}{\rightarrow} \mathcal{M}$ 。 promotes to a symmetric monoidal functor. In particular, the unit of this symmetric monoidal structure $\mathcal{S} \stackrel{u_{\circ}}{\longrightarrow} \mathcal{M}$ 。 is the composition

$$
\mathcal{S} \stackrel{u}{\rightarrow} \mathcal{M} \stackrel{L}{\rightarrow} \mathcal{M}_{\circ}
$$

where $u$ is the unit of the symmetric monoidal structure of $\mathcal{M}$. We need to show that $u_{\circ} \otimes \mathcal{M}_{\circ}$ is an equivalence, or equivalently, that its right adjoint $G_{\circ}$ is an equivalence. Let $\mathcal{M}_{\circ} \otimes \mathcal{M}_{\circ} \stackrel{m_{\circ}}{\longrightarrow} \mathcal{M}_{\circ}$ be the tensor product functor. Since the composition

$$
\mathcal{S} \otimes \mathcal{M}_{\circ} \stackrel{u_{\circ} \otimes \mathcal{M}_{\circ}}{\longrightarrow} \mathcal{M}_{\circ} \otimes \mathcal{M}_{\circ} \stackrel{m_{\circ}}{\longrightarrow} \mathcal{M}_{\circ}
$$


is an equivalence, so is the composition of the right adjoints. It follows that $G_{\circ}$ is essentially surjective. To complete the proof, we shall show that $G_{\circ}$ is also fully faithful. Write $u_{\circ} \otimes \mathcal{M}_{\circ}$ as the composition

$$
\mathcal{S} \otimes \mathcal{M}_{\circ} \stackrel{u \otimes \mathcal{M}_{\circ}}{\longrightarrow} \mathcal{M} \otimes \mathcal{M}_{\circ} \stackrel{L \otimes \mathcal{M}_{\circ}}{\longrightarrow} \mathcal{M}_{\circ} \otimes \mathcal{M}_{\circ} .
$$

Let $G$ be the right adjoint of $u \otimes \mathcal{M}_{\circ}$. It follows that $G_{\circ}$ is the composition of the right adjoint of $L \otimes \mathcal{M}_{\circ}$ and $G$. Since $L$ admits a fully faithful right adjoint, by Lemma 5.2.1, the functor $L \otimes \mathcal{M}$ 。 has a fully faithful right adjoint as well. Thus, it suffices to show that $G$ is fully faithful. For this, consider the commutative diagram

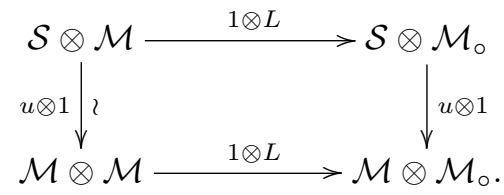

Taking the right adjoints, we see that the composition of $G$ with the right adjoint of $\mathcal{M} \otimes L$, which is fully faithful by Lemma 5.2.1, is fully faithful. It follows that $G$ must be fully faithful as well. This concludes the proof that $u_{\circ}$ exhibits $\mathcal{M}$ as a mode.

Now, we want to analyze the property classified by $\mathcal{M}_{\circ}$. Since $L$ is symmetric monoidal, $\mathcal{M}_{\circ}$ is a commutative algebra over $\mathcal{M}$. Thus, every $\mathcal{M}_{\circ}$-module is, in particular, an $\mathcal{M}$-module. Given an $\mathcal{M}$-module $\mathcal{C}$, it is an $\mathcal{M}_{\circ}$-module if and only if the composition

$$
\mathcal{C} \otimes \mathcal{S} \stackrel{\mathcal{C} \otimes u}{\longrightarrow} \mathcal{C} \otimes \mathcal{M} \stackrel{\mathcal{C} \otimes L}{\longrightarrow} \mathcal{C} \otimes \mathcal{M} \text { 。 }
$$

is an equivalence. The first functor is an equivalence since $\mathcal{C}$ is an $\mathcal{M}$-module. Thus, by 2 -out-of-3, the composition is an equivalence if and only if $\mathcal{C} \otimes L$ is an equivalence. The functor $\mathcal{C} \otimes L$ admits by Lemma 5.2.1 a fully faithful right adjoint. To describe its essential image, we consider the commutative diagram of Yoneda embeddings

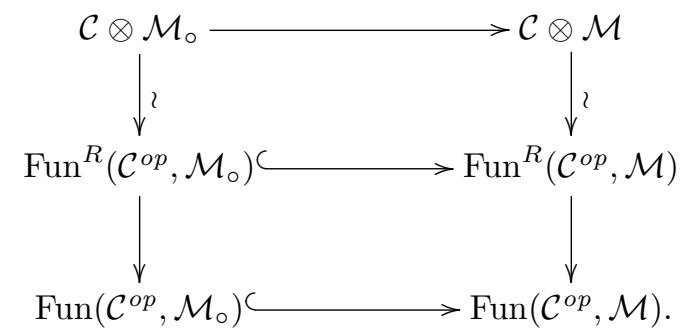

We see that $\mathcal{C} \otimes \mathcal{M}_{\circ}$ is identified with the full subcategory of $\mathcal{M}_{\circ}$-local objects in $\mathcal{C}$.

Remark 5.2.11. Proposition 5.2.10 need not hold for a reflective subcategory $\mathcal{M} \circ \subseteq \mathcal{M}$, which is not assumed in advance to be compatible with the symmetric monoidal structure. Indeed, the inclusion of co-connective spectra $\mathrm{Sp}^{\mathrm{co}-\mathrm{cn}} \subseteq \mathrm{Sp}$ is reflective with a left adjoint $\tau_{\leq 0}$. However, we have

$$
\mathrm{Sp}^{\mathrm{co}-\mathrm{cn}} \otimes \mathrm{Sp}^{\mathrm{co}-\mathrm{cn}} \simeq 0 .
$$

Indeed, $\mathrm{Sp}^{\mathrm{co}-\mathrm{cn}} \otimes \mathrm{Sp}$ is the $\infty$-category of spectrum objects in $\mathrm{Sp}^{\mathrm{co}-\mathrm{cn}}$, and thus is zero. But by Lemma 5.2.1, the functor

$$
0=\mathrm{Sp}^{\mathrm{co}-\mathrm{cn}} \otimes \mathrm{Sp} \stackrel{\mathrm{Id} \otimes \tau_{\leq 0}}{\longrightarrow} \mathrm{Sp}^{\mathrm{co}-\mathrm{cn}} \otimes \mathrm{Sp}^{\mathrm{co}-\mathrm{cn}}
$$


admits a fully faithful right adjoint, so $\mathrm{Sp}^{\mathrm{co}-\mathrm{cn}} \otimes \mathrm{Sp}^{\mathrm{co}-\mathrm{cn}}$ must be zero as well.

Many further examples of modes can be constructed using Proposition 5.2.10:

Example 5.2.12. Consider the subcategory $\mathcal{S}_{\leq d} \subseteq \mathcal{S}$ of $d$-truncated spaces. Every $\mathcal{C} \in \operatorname{Pr}$ is an $\mathcal{S}$ module and an object $X \in \mathcal{C}$ is $\mathcal{S}_{\leq d}$-local if and only if it is $d$-truncated. Thus, $\mathcal{S}_{\leq d}$ is a mode which classifies the property that every object is $d$-truncated. Namely, the property of being equivalent to a $(d+1)$-category (compare with Remark 5.2.11). The cases $d=-2,-1$ and 0 , reproduce the terminal mode 0 , the boolean mode $(0 \rightarrow 1)$, and the discrete mode Set respectively.

Another important family of examples is the Bousfield localizations:

Example 5.2.13. For every $E \in \mathrm{Sp}$, the full subcategory $\mathrm{Sp}_{E} \subseteq \mathrm{Sp}$ of $E$-local spectra is a mode and we have that $\mathrm{Sp}_{E_{1}} \simeq \mathrm{Sp}_{E_{2}}$ in $\mathrm{CAlg}(\mathrm{Pr})$, if and only if $E_{1}$ and $E_{2}$ are Bousfield equivalent. For every $\mathcal{C} \in \operatorname{Pr}_{\text {st }}$, we write $\mathcal{C}_{E}:=\operatorname{Sp}_{E} \otimes \mathcal{C}$ and $\mathcal{C}_{(p)}:=\operatorname{Sp}_{(p)} \otimes \mathcal{C}$ for a prime $p$.

Remark 5.2.14. Given $E_{1}, E_{2} \in \mathrm{Sp}$, if $\mathrm{Sp}_{E_{1}} \otimes \mathrm{Sp}_{E_{2}}=0$, then $\mathrm{Sp}_{E_{1}} \times \mathrm{Sp}_{E_{2}}$ is a mode (Proposition 5.2.5(7)). However, it is usually not a localization of $\mathrm{Sp}$, and in particular, it is not the same as $\mathrm{Sp}_{E_{1} \oplus E_{2}}$. For example, for every $n \geq 1$ we have

$$
L_{n}^{f} \mathrm{Sp} \simeq \mathrm{Sp}_{\oplus_{k=0}^{n} T(k)} \not 千 \prod_{k=0}^{n} \mathrm{Sp}_{T(k)} .
$$

Note that the right-hand side is $\infty$-semiadditive, while the left-hand side is not even 1-semiadditive. In Theorem 5.4.10, we shall show that in a sense, this is the difference between the left and righthand sides.

As with Bousfield localization, a particularly nice kind of localizations of modes, is given by the ones which are smashing in the sense of Definition 5.1.2. The smashing localizations of modes have a very simple characterization:

Proposition 5.2.15. A localization of modes $L: \mathcal{M} \rightarrow \mathcal{N}$ is smashing, if and only if the (fully faithful) right adjoint of $L$ admits a further right adjoint.

Proof. In one direction, the forgetful functor $\operatorname{Mod}_{R}(\mathcal{M}) \rightarrow \mathcal{M}$ admits a right adjoint for every $R \in \mathrm{CAlg}(\mathcal{M})$ by [Lura, Remark 4.2.3.8]. Conversely, by Proposition 5.1.4, it suffices to show that if the right adjoint $F$ of $L$ admits a further right adjoint, the adjunction $F \dashv L$ satisfies the projection formula. Since $F$ is then colimit preserving, the natural transformation in the projection formula

$$
X \otimes F(Y) \stackrel{\alpha}{\rightarrow} F(L(X) \otimes Y)
$$

is a natural transformation between two functors $\mathcal{M} \times \mathcal{N} \rightarrow \mathcal{M}$, which are colimit preserving in each variable. Equivalently, these are colimit preserving functors $\mathcal{M} \otimes \mathcal{N} \rightarrow \mathcal{M}$ [Lura, Section 4.8.1]. Thus, it suffices to check that $\alpha$ becomes an isomorphism after whiskering along the equivalence

$$
\mathcal{N} \simeq \mathcal{S} \otimes \mathcal{N} \stackrel{\sim}{\longrightarrow} \mathcal{M} \otimes \mathcal{N}
$$

This amounts to verifying the case $X=\mathbb{1}_{\mathcal{M}}$, in which, by unwinding the definitions, $\alpha$ is the identity and so in particular an isomorphism. 
Remark 5.2.16. Every mode $\mathcal{M}$ provides by definition a smashing localization $\operatorname{Pr} \rightarrow \operatorname{Mod}_{\mathcal{M}}(\operatorname{Pr})$. Furthermore, every map of modes $\mathcal{M} \rightarrow \mathcal{N}$ induces a smashing localization of the $\infty$-categories of

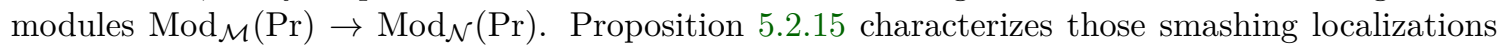
of $\operatorname{Mod}_{\mathcal{M}}(\mathrm{Pr})$, which arise from smashing localizations of $\mathcal{M}$.

A particular instance of mode localizations arises from divisible and complete objects with respect to a natural endomorphism of the identity functor. More generally,

Proposition 5.2.17. Let $\mathcal{C} \in \operatorname{Pr}$ and $\operatorname{Id}_{\mathcal{C}} \stackrel{\alpha,}{\rightarrow} \operatorname{Id}_{\mathcal{C}}$. The $\infty$-categories $\mathcal{C}\left[\alpha^{-1}\right]$ and $\widehat{\mathcal{C}}_{\alpha}$ are accessible localizations of $\mathcal{C}$ and hence in particular presentable. If moreover $\mathcal{C} \in \mathrm{CAlg}(\mathrm{Pr})$, and $\alpha$ is given by tensoring with $\mathbb{1} \stackrel{\alpha_{1}}{\longrightarrow} \mathbb{1}$, then the full subcategories $\mathcal{C}\left[\alpha^{-1}\right], \widehat{\mathcal{C}}_{\alpha} \subseteq \mathcal{C}$ are compatible with the symmetric monoidal structure of $\mathcal{C}$ and are thus symmetric monoidal localizations of $\mathcal{C}$.

Proof. To show that $\mathcal{C}\left[\alpha^{-1}\right]$ and $\widehat{\mathcal{C}_{\alpha}}$ are accessible localizations of $\mathcal{C}$, we use [Lur09, Propostion 5.5.4.15], by which it suffices to realize them as the full subcategories of $S$-local objects with respect to a suitable (small) set of morphisms in $\mathcal{C}$. Since $\mathcal{C}$ is presentable, it is $\kappa$-compactly generated for some cardinal $\kappa$. In particular, the subcategory $\mathcal{C}^{\kappa} \subseteq \mathcal{C}$ of $\kappa$-compact objects is essentially small and generates $\mathcal{C}$ under colimits. For $\mathcal{C}\left[\alpha^{-1}\right]$, we take $S$ to be the collection of maps $X \stackrel{\alpha}{\rightarrow} X$ for $X$ in (a set of representatives of) $\mathcal{C}^{\kappa}$. For $\widehat{\mathcal{C}_{\alpha}}$, we can take $S^{\prime}$ to be the collection of maps $0 \rightarrow Z$ for $Z$ in (a set of representatives of) $\tau$-compact objects for $\tau$ large enough so that $\mathcal{C}\left[\alpha^{-1}\right]$ is $\tau$-compactly generated and the inclusion $\mathcal{C}\left[\alpha^{-1}\right] \hookrightarrow \mathcal{C}$ is $\tau$-accessible.

For $\mathcal{C} \in \mathrm{CAlg}(\operatorname{Pr})$, the assumption on $\alpha$ implies that for all $X, Y \in \mathcal{C}$ we have $\alpha_{X \otimes Y}=X \otimes \alpha_{Y}$ and similarly for the adjoint $\alpha_{\operatorname{hom}(X, Y)}=\operatorname{hom}\left(X, \alpha_{Y}\right)$. In particular, the class of $\alpha$-divisible objects is closed under tensoring and exponentiation by any object of $\mathcal{C}$. Now, the class of morphisms $\bar{S}$ in $\mathcal{C}$, which are mapped to isomorphisms under the localization $\mathcal{C} \rightarrow \mathcal{C}\left[\alpha^{-1}\right]$, is the set of maps $X \stackrel{f}{\rightarrow} Y$ in $\mathcal{C}$ such that for every $W \in \mathcal{C}\left[\alpha^{-1}\right]$, the map

$$
\operatorname{Map}(Y, W) \stackrel{(-) \circ f}{\longrightarrow} \operatorname{Map}(X, W)
$$

is an isomorphism. For any $Z \in \mathcal{C}$, we have that $\operatorname{hom}(Z, W) \in \mathcal{C}\left[\alpha^{-1}\right]$. Thus, by adjointness, $Z \otimes f \in \bar{S}$. The argument for $\widehat{\mathcal{C}}_{\alpha}$ is similar but simpler. It again suffices to show that for $Z \in \mathcal{C}$ and $W \in \widehat{\mathcal{C}}_{\alpha}$, the object $\operatorname{hom}^{\mathcal{C}}(W, Z)$ is $\alpha$-complete. This follows from the fact that for any $\alpha$-divisible $X$, the object $W \otimes X$ is $\alpha$-divisible.

\subsection{Modes of Semiadditivity}

In this subsection, we apply the general theory of modes to study the interaction of stability and higher semiadditivity. In particular, we introduce and study the mode which classifies the property of being stable, $\infty$-semiadditive and of semiadditive height $n$, and compare it with $\operatorname{Sp}_{T(n)}$.

\section{Semiadditivity \& stability}

It is a fundamental result of [Har17], that higher semiadditivity is classified by a mode. More precisely, by Lemma 2.1.15 the forgetful functor

$$
\mathrm{CMon}_{m} \rightarrow \mathrm{CMon}_{-2} \simeq \mathcal{S}
$$


admits a left adjoint $\mathbb{1}_{\mathrm{Pr}}=\mathcal{S} \rightarrow \mathrm{CMon}_{m}$. We consider $\mathrm{CMon}_{m}$ as an object of $\mathrm{Pr}_{\mathbb{1}} /$ via this left adjoint.

Proposition 5.3.1. Let $-2 \leq m \leq \infty$. $\mathrm{CMon}_{m}$ is a mode, which classifies m-semiadditivity. Moreover, for every $\mathcal{C} \in \operatorname{Pr}$, there is a canonical equivalence ${ }^{14}$

$$
\mathrm{CMon}_{m} \otimes \mathcal{C} \simeq \mathrm{CMon}_{m}(\mathcal{C}) .
$$

Proof. We first treat the case $m<\infty$. The fact that $\mathrm{CMon}_{m}$ is a mode, which classifies $m$ semiadditivity is exactly [Har17, Corollary 5.21]. Consider now the inclusion $\iota: \operatorname{Pr}^{\oplus-m} \hookrightarrow \operatorname{Pr}$. To prove that $\mathrm{CMon}_{m} \otimes \mathcal{C} \simeq \mathrm{CMon}_{m}(\mathcal{C})$, note that [Har17, Corollary 5.21] and [Har17, Corollary 5.18] identify the left-hand side and the right-hand side respectively as left adjoints to $\iota$.

We now consider the case $m=\infty$. For verious $k$, the left adjoints of the forgetful functors $\mathrm{CMon}_{k+1} \rightarrow \mathrm{CMon}_{k}$ can be considered as maps in $\mathrm{CAlg}^{\text {idem }}(\mathrm{Pr}) \subseteq \mathrm{Pr}_{1 / 1}$. Thus, by Lemma 2.1.15 and Proposition 5.2.5(5), we have that

$$
\mathrm{CMon}_{\infty} \simeq \lim _{k} \mathrm{CMon}_{k} \in \mathrm{CAlg}^{\text {idem }}(\mathrm{Pr})
$$

is a mode, classifying the property of being $k$-semiadditive for every $k$. In other words, $\mathrm{CMon}_{\infty}$ is a mode classifying $\infty$-semiadditivity. Finally, since $\operatorname{Pr}$ is closed symmetric monoidal, for every $\mathcal{C} \in \operatorname{Pr}$ we have

$$
\mathrm{CMon}_{\infty} \otimes \mathcal{C} \simeq\left(\lim _{k} \mathrm{CMon}_{k}\right) \otimes \mathcal{C} \simeq \lim _{k}\left(\mathrm{CMon}_{k} \otimes \mathcal{C}\right) \simeq \underline{\lim }_{k} \operatorname{CMon}_{k}(\mathcal{C}) \simeq \operatorname{CMon}_{\infty}(C)
$$

Remark 5.3.2. The mere fact that $m$-semiadditivity is classified by a mode has already nontrivial implications. For example, given $\mathcal{C} \stackrel{F}{\rightarrow} \mathcal{D}$ in $\operatorname{Alg}(\operatorname{Pr})$, we get by Proposition 5.2.8, that if $\mathcal{C}$ is $m$-semiadditive then so is $\mathcal{D}$ (compare [CSY18, Corollary 3.3.2(2)]).

To study the interaction of higher semiadditivity and stability we introduce the stable $m$-semiadditive mode:

Definition 5.3.3. For every $0 \leq m \leq \infty$, we define the $\infty$-category of $m$-commutative monoids in spectra:

$$
\mathfrak{צ}^{[m]}:=\mathrm{CMon}_{m}(\mathrm{Sp}) \text {. }
$$

We denote $\boldsymbol{\xi}^{[\infty]}$ simply by $\boldsymbol{צ}$ (and observe that $\boldsymbol{צ}^{[0]} \simeq \mathrm{Sp}$ ).

It is an immediate consequence of Proposition 5.3.1 and Proposition 5.2.5(4), that for all values $0 \leq m \leq \infty$, the $\infty$-category $\mathbf{3}^{[m]}$ is a mode which classifies the property of being $m$-semiadditive and stable.

The mode $\mathbf{3}^{[m]}$ plays an analogous role to $\mathrm{CMon}_{m}$ for stable $m$-semiadditive $\infty$-categories (even for the non-presentable ones). In particular, the $\mathrm{CMon}_{m}$-enriched Yoneda embedding of an $\mathrm{m}$ semiadditive $\infty$-category can be further lifted to $\mathbf{\Xi}^{[m]}$, provided that the $\infty$-category is stable. To

\footnotetext{
${ }^{14}$ This can be compared with the fact that the stabilization $\operatorname{Sp} \otimes \mathcal{C}$ can be identified with $\operatorname{Sp}(\mathcal{C})$, the $\infty$-category of spectrum objects in $\mathcal{C}$.
} 
see this, for a stable $\infty$-category $\mathcal{C}$, we already have a limit preserving spectral Yoneda embedding (e.g. as can be deduced from [Lura, Corollary 1.4.2.23])

$$
\left\llcorner^{\mathrm{Sp}}: \mathcal{C} \hookrightarrow \operatorname{Fun}\left(\mathcal{C}^{o p}, \mathrm{Sp}\right) .\right.
$$

By Proposition 2.1.16, if $\mathcal{C}$ is in addition $m$-semiadditive, we have a canonical equivalence

$$
\left.\operatorname{Fun}^{m \text {-fin }}\left(\mathcal{C}^{o p}, \mathrm{CMon}_{m}(\mathrm{Sp})\right) \simeq \operatorname{Fun}^{m \text {-fin }}\left(\mathcal{C}^{o p}, \mathrm{Sp}\right)\right) .
$$

Thus, we get a $\mathbf{3}^{[\mathrm{m}]}$-enriched Yoneda embedding functor

$$
\left\llcorner^{\mathbf{y}^{[m]}}: \mathcal{C} \rightarrow \operatorname{Fun}^{m-\operatorname{fin}}\left(\mathcal{C}^{o p}, \mathbf{y}^{[m]}\right) \subseteq \operatorname{Fun}\left(\mathcal{C}^{o p}, \mathbf{y}^{[m]}\right) .\right.
$$

We note that this functor is fully faithful, exact and $m$-semiadditive. Using the $\mathbf{3}^{[\mathrm{m}]}$-enriched Yoneda embedding, we can characterize the semiadditive height of an object.

Proposition 5.3.4. Let $\mathcal{C} \in \mathrm{Cat}_{\mathrm{st}}^{\oplus-m}$ and let $X \in \mathcal{C}$. For every $0 \leq n \leq m$, the object $X$ is of height $\leq n($ resp. $>n-1)$ if and only if $\operatorname{hom}^{\mathbf{w}^{[m]}}(Z, X) \in \mathbf{y}^{[m]}$ is of height $\leq n$ (resp. $\left.>n-1\right)$, for every object $Z \in \mathcal{C}$.

Proof. Using the $\mathbf{3}^{[m]}$-enriched Yoneda embedding for $\mathcal{C}^{o p}$, the functors

$$
\operatorname{hom}^{\mathbf{y}^{[m]}}(Z,-): \mathcal{C} \rightarrow \mathbf{y}^{[m]} \quad, \quad Z \in \mathcal{C}
$$

are $m$-semiadditive and jointly conservative. Thus, by Corollary 4.2.3, $X$ is of height $\leq n$ or $>n-1$ if and only if $\operatorname{hom}^{\mathbf{3}^{[m]}}(Z, X)$ is so for all $Z \in \mathcal{C}$.

Another useful application of the $\mathbf{3}^{[\mathrm{m}]}$-enriched Yoneda embedding was already used in the proof of Theorem 4.2.7:

Proposition 5.3.5. For every $\mathcal{C} \in \mathrm{Cat}_{\mathrm{st}}^{\oplus-m}$, there exists $\widehat{\mathcal{C}} \in \operatorname{Pr}_{\mathrm{st}}^{\oplus-m}$ and a fully faithful, $m$ semiadditive and exact embedding $\mathcal{C} \hookrightarrow \widehat{\mathcal{C}}$.

Proof. We take

$$
よ^{\mathbf{y}^{[m]}}: \mathcal{C} \hookrightarrow \operatorname{Fun}\left(\mathcal{C}^{o p}, \mathbf{צ}^{[m]}\right)=: \widehat{\mathcal{C}}
$$

which satisfies all the required properties.

\section{Modes of semiadditive height}

We can further concentrate on stable higher semiadditive $\infty$-categories of particular semiadditive height. We shall now show that this property is also classified by a mode.

Theorem 5.3.6. For every prime $p$ and $0 \leq n \leq \infty$, there exists a mode $\boldsymbol{\vartheta}_{n}$ which classifies the property of being stable p-local $\infty$-semiadditive of height $n^{15}$. Moreover, $\mathbf{\Xi}_{n}$ can be canonically identified with $\mathbf{צ}_{(p), n}^{[m]}$ for every $n \leq m \leq \infty$.

\footnotetext{
${ }^{15}$ We keep $p$ implicit in the notation $\boldsymbol{צ}_{n}$, by analogy with $\operatorname{Sp}_{T(n)}$ and $\operatorname{Sp}_{K(n)}$.
} 
Proof. We first consider the case $n<\infty$. For every $m \geq n$, we have that

$$
\mathbf{\mho}_{(p), n}^{[m]}=\widehat{\left(\mathbf{\mho}_{(p)}^{[m]}\right)}{ }_{p_{(n-1)}}\left[p_{(n)}^{-1}\right]
$$

is a symmetric monoidal localization of $\boldsymbol{צ}_{(p)}^{[m]}$ (Proposition 5.2.17). By Proposition 5.3.4, for every $p$-local $\mathcal{C} \in \operatorname{Pr}_{\text {st }}^{\oplus-m}$, an object $X \in \mathcal{C}$ is $\mathbf{צ}_{(p), n}^{[m]}$-local, if and only if $\operatorname{ht}(X)=n$. Hence, we can apply Proposition 5.2.10 to deduce that $\mathbf{צ}_{(p), n}^{[m]}$ is itself a mode classifying the property of being stable $p$-local $m$-semiadditive and of height $n$. Finally, by Theorem 3.2.7, every such $\infty$-category is $\infty$-semiadditive and hence we can take $\boldsymbol{צ}_{n}$ to be $\boldsymbol{צ}_{(p), n}^{[m]}$.

For $n=\infty$, we have

$$
\mathbf{\mho}_{(p), \infty}=\bigcap_{n \in \mathbb{N}} \boldsymbol{\mho}_{(p),>n} .
$$

Each $\mathbf{צ}_{(p),>n}=\left(\widehat{\boldsymbol{\mathbf { S }}_{(p)}}\right)_{p_{(n)}}$ is a symmetric monoidal localization of $\mathbf{\mho}_{(p)}^{[m]}$ (Proposition 5.2.17). Thus, $\mathbf{\mho}_{(p), \infty}$ is also an accessible reflective subcategory of $\boldsymbol{\mathbf { ~ }}_{(p)}$ ([Lur09, Proposition 5.4.7.10]), which is compatible with the symmetric monoidal structure. It follows from Proposition 5.2.10, that $\boldsymbol{צ}_{(p), \infty}$ is a mode. Moreover, $\boldsymbol{\mho}_{(p), \infty}$ classifies the property of being stable $p$-local $\infty$-semiadditive and of height $>n$ for all $n$, which is the same as being of height $\infty$.

Example 5.3.7. In the case $n=0$, we have

$$
\mathbf{3}_{0} \simeq \mathbf{צ}_{(p), 0}^{[0]} \simeq \mathrm{Sp}_{(p), 0} \simeq \mathrm{Sp}_{\mathbb{Q}} .
$$

As with the $\operatorname{Sp}_{T(n)}$-s, the modes $\boldsymbol{\Xi}_{n}$ are disjoint for different $n$-s.

Proposition 5.3.8. For all $n \neq k$, we have $\mathbf{\Xi}_{n} \otimes \mathbf{צ}_{k}=0$.

Proof. The $\infty$-category $\boldsymbol{צ}_{n} \otimes \boldsymbol{צ}_{k}$ is $\infty$-semiadditive in which every object is of height both $n$ and $k$, and hence must be the zero object.

Another aspect in which $\boldsymbol{צ}_{n}$ resembles $\mathrm{Sp}_{T(n)}$, is that it kills all bounded above spectra:

Proposition 5.3.9. For $1 \leq n<\infty$, the map of modes $\mathrm{Sp} \rightarrow \mathbf{צ}_{n}$ vanishes on all bounded above spectra.

Proof. Denote by $F: \mathrm{Sp} \rightarrow \mathbf{צ}_{n}$ the map of modes. The class of spectra on which $F$ vanishes is closed under colimits and desuspensions in Sp. Hence, by a standard devissage argument, it suffices to show that $F$ vanishes on $\mathbb{Q}$ and $\mathbb{F}_{\ell}$ for all primes $\ell$. First, $\mathbb{Q}$ and $\mathbb{F}_{\ell}$ for $\ell \neq p$ are $p$-divisible. Since $F$ is 0 -semiadditive, $F(\mathbb{Q})$ and $F\left(\mathbb{F}_{\ell}\right)$ are $p$-divisible as well, but all objects of $\boldsymbol{צ}_{n}$ are $p$-complete, and so $F(\mathbb{Q})=F\left(\mathbb{F}_{\ell}\right)=0$. Thus, it remains to show that $F\left(\mathbb{F}_{p}\right)=0$. For every $k \in \mathbb{N}$ we denote by $\overline{\mathbb{S}}\left[B^{k} C_{p}\right]$ the fiber of the fold map $\mathbb{S}\left[B^{k} C_{p}\right] \rightarrow \mathbb{S}$. Applying $F$, we get a fiber sequence

$$
F\left(\overline{\mathbb{S}}\left[B^{k} C_{p}\right]\right) \rightarrow \mathbb{1}_{\mathbf{y}_{n}}\left[B^{k} C_{p}\right] \rightarrow \mathbb{1}_{\mathbf{y}_{n}} .
$$

Since $\boldsymbol{\Xi}_{n}$ is by definition of height $n$, it follows from Proposition 3.2.3(2), that the second map above is an isomorphism for $k \geq n+1$ and hence $F\left(\overline{\mathbb{S}}\left[B^{k} C_{p}\right]\right)=0$. We observe that $\mathbb{F}_{p}$ can be written as a filtered colimit $\mathbb{F}_{p}=\lim \Sigma^{-k} \overline{\mathbb{S}}\left[B^{k} C_{p}\right]$. Thus, we also get

$$
F\left(\mathbb{F}_{p}\right)=\lim _{\longrightarrow} \Sigma^{-k} F\left(\overline{\mathbb{S}}\left[B^{k} C_{p}\right]\right)=0 .
$$


Corollary 5.3.10. For $1 \leq n<\infty$, the right adjoint of the map of modes $\mathcal{S} \rightarrow \boldsymbol{צ}_{n}$ is conservative.

Proof. The map of modes $\mathcal{S} \rightarrow \mathbf{צ}_{n}$ is given by the composition

$$
\mathcal{S} \stackrel{\mathbb{S}[-]}{\longrightarrow} \operatorname{Sp} \stackrel{F_{1}}{\longrightarrow} \operatorname{Sp}_{(p)} \stackrel{F_{2}}{\longrightarrow} \mathbf{\mho}_{(p)}^{[n]} \stackrel{F_{3}}{\longrightarrow} \mathbf{\mho}_{n} .
$$

The right adjoints $G_{1}$ and $G_{3}$ of $F_{1}$ and $F_{3}$ respectively, are fully faithful embeddings and hence in particular conservative. The right adjoint $G_{2}$ of $F_{2}$ can be identified with the functor

$$
\mathbf{y}_{(p)}^{[n]}=\mathrm{CMon}_{n}\left(\operatorname{Sp}_{(p)}\right) \rightarrow \operatorname{Sp}_{(p)}
$$

which evaluates at the point. Thus, $G_{2}$ is conservative by [Har17, Lemma 5.17]. Combining the above, the right adjoint of the functor

$$
F=F_{3} F_{2} F_{1}: \mathrm{Sp} \rightarrow \boldsymbol{צ}_{n}
$$

is given by $G=G_{1} G_{2} G_{3}$, and is therefore conservative.

Now, the right adjoint of $\mathcal{S} \rightarrow \boldsymbol{צ}_{n}$, is given by the composition of the right adjoints $\boldsymbol{צ}_{n} \stackrel{G}{\longrightarrow}$ Sp $\stackrel{\Omega^{\infty}}{\longrightarrow} \mathcal{S}$. Let $X \stackrel{f}{\rightarrow} Y$ be a map in $\boldsymbol{\mho}_{n}$ with fiber $Z$, such that $\Omega^{\infty} G(f)$ is an isomorphism. It follows that $\Omega^{\infty} G(Z)=0$ and hence $G(Z)$ is co-connective. By Proposition 5.3.9, we get $F G(Z)=0$ and hence $G F G(Z)=0$. By the zig-zag identities, $G(Z)$ is a retract of $G F G(Z)$ and hence we also get $G(Z)=0$. Finally, since $G$ is conservative, we get $Z=0$ and hence $X \stackrel{f}{\rightarrow} Y$ is an isomorphism. This concludes the proof that $\Omega^{\infty} G$, the right adjoint of $\mathcal{S} \rightarrow \boldsymbol{צ}_{n}$, is conservative.

Remark 5.3.11. Conjecture 4.2 .8 is equivalent to the statement that $\boldsymbol{צ}_{\infty}=0$.

\subsection{1-Semiadditive Decomposition}

As we recalled in Example 4.1.3, while for $n \geq 1$ the $\infty$-category $L_{n}^{f}$ Sp itself is not even 1semiadditive, it is an iterated recollement of the $\infty$-categories $\operatorname{Sp}_{T(k)}$ for $k=0, \ldots, n$, which are $\infty$-semiadditive. The theory of modes allows us to enforce $m$-semiadditivity on $L_{n}^{f} \mathrm{Sp}$ in a universal way, by tensoring it with the $m$-semiadditive mode $\mathrm{CMon}_{m}$. We shall show that enforcing even 1-semiadditivity on $L_{n}^{f} \mathrm{Sp}$, "dissolves the glue" which holds the monochromatic layers together, and decomposes it into a product of $\mathrm{Sp}_{T(k)}$ for $k=0, \ldots, n$.

Remark 5.4.1. The fact that $L_{n}^{f} \mathrm{Sp}$ is a recollement of $\mathrm{Sp}_{T(k)}$ for $k=0, \ldots, n$, is closely related to the fact that for $k_{0}<k_{1}$, every exact functor $\mathrm{Sp}_{T\left(k_{0}\right)} \rightarrow \mathrm{Sp}_{T\left(k_{1}\right)}$ must be zero. The fact that the recollement becomes split after imposing 1-semiadditivity follows from the fact that every 1semiadditive functor $\mathrm{Sp}_{T\left(k_{1}\right)} \rightarrow \mathrm{Sp}_{T\left(k_{0}\right)}$ must be zero (Corollary 5.4.9). Note that by Corollary 4.2.3, a $k_{1}$-semiadditive functor $\mathrm{Sp}_{T\left(k_{1}\right)} \rightarrow \mathrm{Sp}_{T\left(k_{0}\right)}$ must be zero because every object of $\mathrm{Sp}_{T\left(k_{1}\right)}$ is of height $k_{1}$, and thus must be sent to the only object of height $k_{1}$ in $\mathrm{Sp}_{T\left(k_{0}\right)}$, which is zero. The main "non-formal" ingredient in the proof of Theorem 5.4.10 (which makes essential use of the theory developed in [CSY18, Section 4]) is that it suffices to assume merely 1-semiadditivity. 


\section{Divisible and complete cardinalities}

We begin with some general observations and constructions. Every $\mathcal{C} \in \operatorname{Pr}_{\mathrm{st}}^{\oplus-1}$ is a module over $\mathbf{y}^{[1]}$ and thus, every $\alpha \in \pi_{0}\left(\mathbb{1}_{\mathfrak{Y}^{[1]}}\right)$ induces a natural transformation $\operatorname{Id}_{\mathcal{C}} \stackrel{\alpha_{\mathcal{C}}}{\longrightarrow} \operatorname{Id}_{\mathcal{C}}$. If in addition $\mathcal{C}$ is presentably symmetric monoidal, then there is a unique functor $\mathbf{y}^{[1]} \rightarrow \mathcal{C}$ in $\operatorname{CAlg}(\operatorname{Pr}$ ) (see Proposition 5.1.6), which induces a map $\pi_{0}\left(\mathbb{1}_{\mathfrak{Y}\left[{ }^{[1]}\right.}\right) \rightarrow \pi_{0}\left(\mathbb{1}_{\mathcal{C}}\right)$. The natural transformation $\alpha_{\mathcal{C}}$ can be identified with the one induced by the image of $\alpha$ in $\pi_{0}\left(\mathbb{1}_{\mathcal{C}}\right)$. We now restrict to a particular subset of $\pi_{0}\left(\mathbb{1}_{\mathbf{Y}[1]}\right)$ consisting of elements which have a simple description, as they are of an unstable origin.

Definition 5.4.2. Let $\mathcal{R}_{1} \subseteq \pi_{0}\left(\mathbb{1}_{\mathbf{y}[1]}\right)$ be the $\mathbb{Z}$-linear span of elements of the form $|B G| \in \pi_{0}\left(\mathbb{1}_{\mathbf{y}}[1]\right)$, for $G$ a finite group.

We observe that the action of $\mathcal{R}_{1}$ on the objects of any stable 1 -semiadditive $\infty$-category $\mathcal{C}$ is natural with respect to 1 -semiadditive functors.

Proposition 5.4.3. Let $F: \mathcal{C} \rightarrow \mathcal{D}$ be a 1-semiadditive functor between stable presentable 1semiadditive $\infty$-categories. For every $\alpha \in \mathcal{R}_{1}$, we have $F\left(\alpha_{\mathcal{C}}\right)=\alpha_{\mathcal{D}}$.

Proof. By 0-semiadditivity, it suffices to consider the case $\alpha=|B G|$ for $G$ a finite group, which follows from the fact that $F$ is 1 -semiadditive.

Remark 5.4.4. If $F$ is further assumed to be colimit preserving, then $F\left(\alpha_{\mathcal{C}}\right)=\alpha_{\mathcal{D}}$ for all elements $\alpha \in \pi_{0}\left(\mathbb{1}_{\mathbf{Y}[1]}\right)$. However, we shall be interested in applying Proposition 5.4.3 to functors $F$, which are not a priori colimit preserving (e.g. right adjoints).

Recall from [CSY18, Theorem 4.3.2], that for every 1-semiadditive stable presentably symmetric monoidal $\infty$-category $\mathcal{C}$, the ring $\pi_{0}\left(\mathbb{1}_{\mathcal{C}}\right)$ is equipped with a canonical additive $p$-derivation

$$
\delta: \pi_{0}\left(\mathbb{1}_{\mathcal{C}}\right) \rightarrow \pi_{0}\left(\mathbb{1}_{\mathcal{C}}\right)
$$

Proposition 5.4.5. The subset $\mathcal{R}_{1} \subseteq \pi_{0}\left(\mathbb{1}_{\mathbf{Y}[1]}\right)$ is closed under multiplication and the additive $p$ derivation $\delta$ inside $\pi_{0}\left(\mathbb{1}_{\mathbf{Y}^{[1]}}\right)$. Consequently, $\mathcal{R}_{1}$ is a semi- $\delta$-ring and the inclusion $\mathcal{R}_{1} \hookrightarrow \pi_{0}\left(\mathbb{1}_{\mathbf{Y}}{ }^{[1]}\right)$ is a homomorphism of semi- $\delta$-rings.

Proof. The closure under multiplication follows from the identity (Remark 2.1.10)

$$
|B G||B H|=|B(G \times H)|
$$

and the closure under $\delta$ follows from the identity

$$
\delta|B G|=\left|B G \curlywedge C_{p}\right|-\left|B\left(C_{p} \times G\right)\right|,
$$

the formula (see [CSY18, Definition 4.1.1(1)])

$$
\delta(x+y)=\delta(x)+\delta(y)+\frac{x^{p}+y^{p}-(x+y)^{p}}{p}
$$

and the closure of $\mathcal{R}_{1}$ under multiplication. 


\section{1-semiadditive decomposition}

We would now like to apply the above to the $\infty$-categories $\operatorname{Sp}_{T(n)}$. By [CSY18, Proposition 4.3.4], the construction of the additive $p$-derivation $\delta$ is functorial with respect to colimit preserving symmetric monoidal functors. In particular, assuming that $n \geq 1$, both maps

$$
\pi_{0}\left(\mathbb{1}_{\mathbf{Y}^{[1]}}\right) \rightarrow \pi_{0} \mathbb{S}_{T(n)} \stackrel{u}{\rightarrow} \pi_{0} E_{n}
$$

commute with $\delta$. By Proposition 2.2.6, the image of $u$ is the canonical $\mathbb{Z}_{p}$ copy obtained from the map

$$
\mathbb{Z}_{p} \simeq \pi_{0} \widehat{\mathbb{S}}_{p} \hookrightarrow \pi_{0} E_{n}
$$

Since $\mathbb{Z}_{p} \subset \pi_{0} E_{n}$ is the image of a semi- $\delta$-ring map we obtain a surjective map of semi- $\delta$-rings

$$
u: \pi_{0} \mathbb{S}_{T(n)} \rightarrow \mathbb{Z}_{p}
$$

The semi- $\delta$-ring structure on $\mathbb{Z}_{p}$ can be explicitly described.

Lemma 5.4.6. The unique semi- $\delta$-ring structure on $\mathbb{Z}_{p}$ is given by

$$
\delta(a)=\frac{a-a^{p}}{p}, \quad \forall a \in \mathbb{Z}_{p}
$$

In particular, if $v_{p}(a)>0$, then $v_{p}(\delta(a))=v_{p}(a)-1$.

Proof. For $a \in \mathbb{Z}_{p}$ we define $\phi(a)=\frac{a-a^{p}}{p}-\delta(a)$. By [CSY18, Definition 4.1.1(1)], the function $\phi: \mathbb{Z}_{p} \rightarrow \mathbb{Z}_{p}$ is additive. By [CSY18, Proposition 4.1.11], $\phi$ factors trough a map $\mathbb{Z}_{p} / \mathbb{Z}_{(p)} \rightarrow \mathbb{Z}_{p}$. Since $\mathbb{Z}_{p} / \mathbb{Z}_{(p)}$ is $p$-divisible, all such maps are zero.

For $a \in \pi_{0} \mathbb{S}_{T(n)}$, we denote by $v_{p}(a)$ the $p$-adic valuation of $u(a) \in \mathbb{Z}_{p} \subseteq \pi_{0} E_{n}$.

Proposition 5.4.7. For every $n \geq 1$ and $a \in \pi_{0} \mathbb{S}_{T(n)}$ we have:

(1) If $v_{p}(a)=0$, then $\mathrm{Sp}_{T(n)}$ is a-divisible.

(2) If $v_{p}(a)>0$, then $\mathrm{Sp}_{T(n)}$ is a-complete.

Proof. For (1), if $v_{p}(a)=0$, then $u(a) \in \pi_{0} E_{n}$ is invertible. It follows that $a \in \pi_{0} \mathbb{S}_{T(n)}$ is invertible by nil-conservativity [CSY18, Corollary 5.1.17]. For (2), let $a \in \pi_{0} \mathbb{S}_{T(n)}$ such that $v_{p}(a)>0$. We need to show that for every $0 \neq X \in \mathrm{Sp}_{T(n)}$, the element $a$ acts non-invertibly on $X$. Since tensoring with $T(n)$ is conservative, we may replace $X$ by $T(n) \otimes X$. Without loss of generality, we may choose $T(n)$ to be a ring spectrum. Thus, $T(n) \otimes X$ is a $T(n)$-module and the action of $a$ on it is by its image under the map $\pi_{0} \mathbb{S}_{T(n)} \rightarrow \pi_{0} T(n)$, which we denote by $\bar{a}$. Thus, it would suffice to show that $\bar{a} \in \pi_{0} T(n)$ is nilpotent. By the Nilpotence Theorem, it suffices to show that the image of $\bar{a}$ under the map

$$
\pi_{0} T(n) \rightarrow \pi_{0}\left(T(n) \otimes E_{n}\right)
$$

is nilpotent, as $T(n) \otimes K(j)=0$ for all $j=n+1, \ldots, \infty$. Consider the commutative diagram

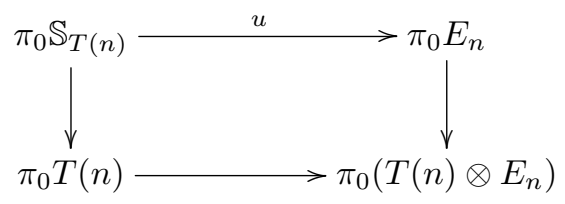


We see that the image of $\bar{a}$ in $\pi_{0}\left(T(n) \otimes E_{n}\right)$ is contained in the image of $u$, which lies in $\mathbb{Z}_{p} \subseteq \pi_{0} E_{n}$ (Proposition 2.2.6). Since $T(n)$ is $p$-power torsion, the map

$$
\mathbb{Z}_{p} \subseteq \pi_{0} E_{n} \rightarrow \pi_{0}\left(T(n) \otimes E_{n}\right)
$$

factors through a finite quotient $\mathbb{Z}_{p} \rightarrow \mathbb{Z} / p^{r}$ and hence the image of $\bar{a}$ in $\pi_{0}\left(T(n) \otimes E_{n}\right)$ is nilpotent.

Proposition 5.4.8. For every $k \geq 0$, there exists an element $\alpha \in \mathcal{R}_{1}$, such that $\operatorname{Sp}_{T(n)}$ is $\alpha$ complete for $n \leq k$ and $\alpha$-divisible for $n>k$.

Proof. First, we observe that it suffices to construct elements $\beta_{(k)}$ such that $\mathrm{Sp}_{T(k)}$ is $\beta_{(k)}$-complete and $\operatorname{Sp}_{T(n)}$ is $\beta_{(k)}$-divisible for $n>k$. Indeed, we can then define $\alpha=\beta_{(0)} \cdots \beta_{(k)}$, which satisfies the required properties. To construct $\beta:=\beta_{(k)}$ for a specific $k \geq 0$, we proceed as follows. For $k=0$, we take $\beta=p\left|B C_{p}\right|-1$. We get that for $\mathrm{Sp}_{T(0)}=\mathrm{Sp}_{\mathbb{Q}}$, the element $\beta$ acts as zero, while for $n>0$, the $\infty$-category $\operatorname{Sp}_{T(n)}$ is $p$-complete, and hence by Proposition 4.1.14, it is $\beta$-divisible. Now, we treat the case $k \geq 1$. For every $a \in \mathcal{R}_{1} \subseteq \pi_{0}\left(\mathbb{1}_{\mathbf{y}^{[1]}}\right)$, we shall denote by $a_{n}$ its image in $\mathbb{Z}_{p} \subseteq \pi_{0} E_{n}$ under the map $\pi_{0}\left(\mathbb{1}_{\mathbf{y}}[1]\right) \rightarrow \pi_{0} E_{n}$. By Proposition 5.4.7, it suffices to construct $\beta$, such that $v_{p}\left(\beta_{k}\right)>0$ and $v_{p}\left(\beta_{n}\right)=0$ for all $n \geq k+1$. Using Proposition 5.4.5, we define the element

$$
\gamma=\delta^{k-1}\left|B C_{p}\right| \in \mathcal{R}_{1} .
$$

By Proposition 2.2.5, we have $\left|B C_{p}\right|_{n}=p^{n-1}$ for all $n \in \mathbb{N}$. Thus, by Lemma 5.4.6, we have $v_{p}\left(\gamma_{n}\right)=n-k$ for all $n \geq k$, and in particular $v_{p}\left(\gamma_{k}\right)=0$. It follows that there exists an integer $b \in \mathbb{Z}$ with $v_{p}(b)=0$, such that $v_{p}\left(\gamma_{k}-b\right)>0$. We define $\beta:=\gamma-b \in \mathcal{R}_{1}$. On the one hand, we have by construction $v_{p}\left(\beta_{k}\right)>0$, and on the other, $v_{p}\left(\beta_{n}\right)=0$ for $n>k$ by the ultrametric property of the $p$-adic valuation.

Corollary 5.4.9. For all $n>k \geq 0$, every 1-semiadditive functor $F: \operatorname{Sp}_{T(n)} \rightarrow \operatorname{Sp}_{T(k)}$ is zero.

Proof. By Proposition 5.4.8, there exists an element $a \in \mathcal{R}_{1}$, such that $\operatorname{Sp}_{T(n)}$ is a-divisible and $\mathrm{Sp}_{T(k)}$ is $a$-complete. Since $F$ is 1-semiadditive, by Proposition 5.4.3, it must preserve the action of $a$. It follows that for every $X \in \mathrm{Sp}_{T(n)}$, the object $F(X)$ is both $a$-divisible and $a$-complete and hence must be zero.

We are now ready to prove that 1 -semiadditivity forces $L_{n}^{f}$ Sp to decompose into its monochromatic pieces.

Theorem 5.4.10 (1-Semiadditive Decomposition). For every $n \geq 0$, there is an equivalence of modes

$$
\mathrm{CMon}_{1} \otimes L_{n}^{f} \mathrm{Sp} \simeq \prod_{k=0}^{n} \mathrm{Sp}_{T(k)} .
$$

Proof. For $n=0$, the claim is $\mathrm{CMon}_{1} \otimes \mathrm{Sp}_{\mathbb{Q}} \simeq \mathrm{Sp}_{\mathbb{Q}}$, which is true because $\mathrm{Sp}_{\mathbb{Q}}$ is 1-semiadditive. It thus suffices to prove by induction on $n \in \mathbb{N}$, that the functor

$$
L_{n}^{f} \mathrm{Sp} \rightarrow L_{n-1}^{f} \mathrm{Sp} \times \mathrm{Sp}_{T(n)},
$$


given by the product of the respective symmetric monoidal localizations, becomes an equivalence after tensoring with $\mathrm{CMon}_{1}$. We know that $L_{n}^{f}$ Sp is a recollement of $L_{n-1}^{f} \mathrm{Sp} \subseteq L_{n}^{f} \mathrm{Sp}$ and $\mathrm{Sp}_{T(n)}$. It follows that $\mathrm{CMon}_{1} \otimes L_{n}^{f} \mathrm{Sp}$ is a recollement of $\mathrm{CMon}_{1} \otimes L_{n-1}^{f} \mathrm{Sp}$ and $\mathrm{CMon}_{1} \otimes \mathrm{Sp}_{T(n)}=\mathrm{Sp}_{T(n)}$ (Proposition 5.2.3). By the inductive hypothesis, we have

$$
\mathrm{CMon}_{1} \otimes L_{n-1}^{f} \mathrm{Sp} \simeq \prod_{k=0}^{n-1} \mathrm{Sp}_{T(k)} .
$$

Thus, $\mathrm{CMon}_{1} \otimes L_{n}^{f} \mathrm{Sp}$ is a recollement of $\prod_{k=0}^{n-1} \mathrm{Sp}_{T(k)}$ and $\mathrm{Sp}_{T(n)}$. It is therefore suffices to show that the gluing data given by the functor

$$
L: \operatorname{Sp}_{T(n)} \rightarrow \prod_{k=0}^{n-1} \operatorname{Sp}_{T(k)}
$$

is zero (Proposition 4.1.4). We observe that $L$ is given as a composition of a right and a left adjoint between 1 -semiadditive $\infty$-categories and hence is 1 -semiadditive. Thus, by Corollary 5.4.9, we must have $L=0$ and hence the corresponding recollement is split. To conclude, the localization functors $L_{n}^{f} \mathrm{Sp} \rightarrow \mathrm{Sp}_{T(k)}$ for $k=0, \ldots, n$ induce a functor $L_{n}^{f} \mathrm{Sp} \rightarrow \prod_{k=0}^{n} \mathrm{Sp}_{T(k)}$, which becomes an equivalence after tensoring with $\mathrm{CMon}_{1}$. In particular, this is a symmetric monoidal equivalence and hence an equivalence of modes.

Remark 5.4.11. By tensoring Theorem 5.4 .10 with $L_{n} \mathrm{Sp}$, we also get

$$
\mathrm{CMon}_{1} \otimes L_{n} \mathrm{Sp} \simeq \prod_{k=0}^{n} \mathrm{Sp}_{K(k)} .
$$

\subsection{Semiadditive vs. Stable Height}

As we recalled in the introduction, for every $n \geq 0$, the localization functors of spectra $L_{n}^{f}, L_{F(n)}$ and $L_{T(n)}$ can be thought of as restriction to heights $\leq n, \geq n$ and $n$ respectively, as measured by the $v_{n}$-self maps. It is natural to compare this notion of height with the semiadditive one considered in this paper. In this subsection, we phrase the notion of height classified by $L_{n}^{f}, L_{F(n)}$ and $L_{T(n)}$ (which for disambiguation we shall call stable height) in the language of modes and establish some comparison results with the notion of semiadditive height. Using that, we shall prove the bounded version of the "Bootstrap Conjecture" (Theorem E), regarding 1-semiadditivity vs. $\infty$-semiadditivity for stable presentable $\infty$-categories.

\section{Stable Height}

By Example 5.2.13, the $\infty$-categories $L_{n}^{f} \mathrm{Sp}, \mathrm{Sp}_{F(n)}$ and $\mathrm{Sp}_{T(n)}$ are themselves modes. Our first goal is to show that the properties classified by them can be profitably reinterpreted in terms of the following notion:

Definition 5.5.1. Given a stable $\infty$-category $\mathcal{C}$, for every $X \in \mathcal{C}$, we define and denote the stable height of $X$ as follows: 
(1) $\operatorname{ht}_{\mathrm{st}}(X) \leq n$, if $F(n+1) \otimes X=0$ for some (hence any) finite spectrum $F(n+1)$ of type $n+1$.

(2) $\operatorname{ht}_{\mathrm{st}}(X)>n$, if $\operatorname{Map}(Z, X) \simeq$ pt for every $Z$ of stable height $\leq n$.

(3) $\mathrm{ht}_{\mathrm{st}}(X)=n$, if ht $\mathrm{st}(X) \leq n$ and $\mathrm{ht}_{\mathrm{st}}(X)>n-1$.

By convention, $\operatorname{ht}_{\mathrm{st}}(X)>-1$ for all $X$, and $\mathrm{ht}_{\mathrm{st}}(X) \leq-1$ if and only if $X=0$. We also extend the definition to $n=\infty$ as follows: For every $X \in \mathcal{C}$, we write $h_{\mathrm{st}}(X)=\infty$ if and only if $\operatorname{ht}_{\mathrm{st}}(X)>n$ for all $n$.

Remark 5.5.2. Since $F(n+2)$ can be constructed as a cofiber of a self map of $F(n+1)$, it is clear that ht $\mathrm{st}_{\mathrm{st}}(X) \leq n$ implies ht $\mathrm{st}_{\mathrm{st}}(X) \leq n+1$. Consequently, ht $\mathrm{st}_{\mathrm{st}}(X)>n$ also implies ht $\mathrm{st}_{\mathrm{st}}(X)>n-1$.

As with the semiadditive height, it is useful to consider the corresponding subcategories of objects of stable height in a certain range:

Definition 5.5.3. Let $\mathcal{C} \in \mathrm{Cat}_{\text {st }}$ and let $0 \leq n \leq \infty$. We denote by $\mathcal{C}_{<{ }^{\text {st }} n}, \mathcal{C}_{>^{\text {st }} n}$, and $\mathcal{C}_{n^{\text {st }}}$ the full subcategories of $\mathcal{C}$ spanned by objects of stable height $\leq n,>n$, and $n$. In addition, we write $\mathrm{Ht}_{\mathrm{st}}(\mathcal{C}) \leq n,>n$, or $n$, if $\mathcal{C}=\mathcal{C}_{\leq^{\text {st }} n}, \mathcal{C}_{>^{\text {st }} n}$, or $\mathcal{C}_{n^{\text {st }}}$ respectively.

Example 5.5.4. In the special case $\mathcal{C}=\mathrm{Sp}_{(p)}$, we have by definition

$$
\mathrm{Sp}_{(p), \leq^{\text {st }} n}=L_{n}^{f} \mathrm{Sp}, \quad \mathrm{Sp}_{(p),>{ }^{\mathrm{st}} n-1}=\mathrm{Sp}_{F(n)}, \quad \mathrm{Sp}_{(p), n^{\text {st }}}=\mathrm{Sp}_{T(n)} .
$$

We note that the subcategory $\operatorname{Sp}_{(p), \infty} \subseteq$ Sp is rather large. First, for every $X \in L_{n}^{f}$ Sp, we have

$$
L_{n}^{f} X \otimes \mathbb{F}_{p} \simeq L_{n}^{f} X \otimes L_{n}^{f} \mathbb{F}_{p} \simeq L_{n}^{f} X \otimes 0 \simeq 0 .
$$

Therefore, for every $\mathbb{F}_{p}$-module spectrum $M$, we have

$$
\operatorname{Map}_{\mathrm{Sp}_{\mathrm{p}}}\left(L_{n}^{f} X, M\right) \simeq \operatorname{Map}_{\operatorname{Mod}_{\mathbb{F}_{p}}(\mathrm{Sp})}\left(\mathbb{F}_{p} \otimes L_{n}^{f} X, M\right) \simeq \operatorname{Map}_{\operatorname{Mod}_{\mathbb{F}_{p}}(\mathrm{Sp})}(0, M) \simeq 0,
$$

which implies that $M \in \operatorname{Sp}_{(p), \infty \text { st }}$. Since the subcategory $\operatorname{Sp}_{(p), \infty \text { st }} \subseteq \operatorname{Sp}$ is closed under limits, it contains all bounded below $p$-complete spectra. In contrast, the $\infty$-category $\mathcal{C}=\prod_{n \in \mathbb{N}} \operatorname{Sp}_{T(n)}$ satisfies $\mathcal{C}_{\infty^{s t}}=0$, even though it contains many objects of unbounded height.

We now show that the modes $L_{n}^{f} \mathrm{Sp}, \mathrm{Sp}_{F(n)}$ and $\mathrm{Sp}_{T(n)}$ classify the properties of having the corresponding stable height.

Proposition 5.5.5. For every $0 \leq n<\infty$, the modes $L_{n}^{f} \mathrm{Sp}, \mathrm{Sp}_{F(n)}$ and $\mathrm{Sp}_{T(n)}$ classify the properties of being stable $p$-local of stable height $\leq n,>n-1$ and $n$ respectively.

Proof. We begin with $L_{n}^{f}$ Sp. It follows from Proposition 5.2.10, that $L_{n}^{f}$ Sp is a mode, which classifies stable $L_{n}^{f}$ Sp-local $\infty$-categories. Thus, it suffice to show that an object $X$ in a stable $\infty$-category $\mathcal{C}$ is $L_{n}^{f}$ Sp-local, if and only if $\mathrm{ht}_{\mathrm{st}}(X) \leq n$. By definition, $X$ is $L_{n}^{f}$ Sp-local, if for every $Z \in \mathrm{Sp}$, the mapping spectrum $\operatorname{hom}_{\mathcal{C}}^{\mathrm{Sp}}(Z, X)$ belongs to $L_{n}^{f} \mathrm{Sp}$. Since the corepresentable functor $\operatorname{hom}_{\mathcal{C}}^{\mathrm{Sp}}(Z,-): \mathcal{C} \rightarrow$ Sp is exact, we have a canonical isomorphism

$$
\operatorname{hom}_{\mathcal{C}}^{\mathrm{Sp}}(Z, F(n+1) \otimes X) \simeq F(n+1) \otimes \operatorname{hom}_{\mathcal{C}}^{\mathrm{Sp}}(Z, X) .
$$


It follows that if ht $\mathrm{st}_{\mathrm{st}}(X) \leq n$, then $X$ is $L_{n}^{f}$ Sp-local. Since the collection of functors $\operatorname{hom}_{\mathcal{C}}^{\mathrm{Sp}}(Z,-)$ for all $Z \in \mathcal{C}$ is also jointly conservative, the converse holds as well.

We now move to $\operatorname{Sp}_{F(n)}$. We first show that if $\operatorname{ht}_{\mathrm{st}}(X)>n-1$, then $\operatorname{hom}_{\mathcal{C}}^{\mathrm{Sp}}(Z, X)$ is $F(n)$-local for all $Z \in \mathcal{C}$. For $A$ an $F(n)$-acyclic spectrum, $A \otimes Z$ is $F(n)$-acyclic as well and hence ht st $(A \otimes Z) \leq n-1$. Since $\mathrm{ht}_{\mathrm{st}}(X)>n-1$, it follows that

$$
\operatorname{Map}_{\mathrm{Sp}}\left(A, \operatorname{hom}_{\mathcal{C}}^{\mathrm{Sp}}(Z, X)\right) \simeq \operatorname{Map}_{\mathcal{C}}(A \otimes Z, X) \simeq \mathrm{pt},
$$

and hence $X$ is $\operatorname{Sp}_{F(n)}$-local. Conversely, assume that $X$ is $\operatorname{Sp}_{F(n)}$-local, and let $Z \in \mathcal{C}$ such that $\mathrm{ht}_{\mathrm{st}}(Z) \leq n-1$. We have

$$
0 \simeq \operatorname{hom}_{\mathcal{C}}^{\mathrm{Sp}}(F(n) \otimes Z, X) \simeq \mathbb{D} F(n) \otimes \operatorname{hom}_{\mathcal{C}}^{\mathrm{Sp}}(Z, X),
$$

where $\mathbb{D} F(n)$ is the Spanier-Whithead dual of $F(n)$, which is itself of type $n$. Since $\operatorname{hom}_{\mathcal{C}}^{\mathrm{Sp}}(Z, X)$ is $F(n)$-local and hence $\mathbb{D} F(n)$-local, we get $\operatorname{hom}_{\mathcal{C}}^{\mathrm{Sp}}(Z, X)=0$. This implies that $\operatorname{Map}_{\mathcal{C}}(Z, X)=\mathrm{pt}$ and so ht $\mathrm{st}_{\mathrm{t}}(X)>n-1$.

Finally, for $\operatorname{Sp}_{T(n)}$ we observe that a spectrum is $T(n)$-local if and only if it is both $F(n)$-local and $\bigoplus_{k=0}^{n} T(k)$-local. Thus,

$$
\mathrm{Sp}_{T(n)} \simeq L_{n}^{f} \mathrm{Sp} \otimes \mathrm{Sp}_{F(n)} .
$$

Hence, it classifies the property of being stable $p$-local of both stable height $\leq n$ and $>n-1$, i.e exactly $n$.

Similarly, we also treat the case of stable height $\infty$.

Corollary 5.5.6. The $\infty$-category $\operatorname{Sp}_{(p), \infty \text { st }}$ is a mode, which classifies the property of being stable of stable height $\infty$.

Proof. We have

$$
\operatorname{Sp}_{(p), \infty \text { st }}=\bigcap_{n \in \mathbb{N}} \operatorname{Sp}_{F(n)} \subseteq \operatorname{Sp}_{(p)},
$$

and thus is an accessible reflective subcategory of $\mathrm{Sp}_{(p)}$, which is compatible with the symmetric monoidal structure [Lur09, Proposition .5.4.7.10]. It follows from Proposition 5.2.10, that $\operatorname{Sp}_{(p), \infty^{s t}}$ is a mode. Moreover, $\mathrm{Sp}_{(p), \infty}$ classifies the property that the $\infty$-category is stable such that every object $X$ is $\operatorname{Sp}_{F(n)}$-local for all $n \in \mathbb{N}$. By Proposition 5.5.5, the said condition on $X$ is equivalent to ht $\mathrm{st}_{\mathrm{st}}(X) \geq n$ for all $n$, which is the same as ht $\mathrm{st}_{\mathrm{st}}(X)=\infty$. Thus, $\operatorname{Sp}_{(p), \infty \text { st }}$ classifies the property of being stable of stable height $\infty$.

Proposition 5.5.5 has the following immediate corollaries regarding stable height for stable presentable $\infty$-categories:

Corollary 5.5.7. For every $p$-local $\mathcal{C} \in \operatorname{Pr}_{\text {st }}$, we have canonical equivalences

$$
\mathcal{C}_{\leq^{\text {st }} n} \simeq \mathcal{C} \otimes L_{n}^{f} \mathrm{Sp}, \quad \mathcal{C}_{>\text {st } n} \simeq \mathcal{C} \otimes \operatorname{Sp}_{F(n+1)}, \quad \mathcal{C}_{n^{\text {st }}} \simeq \mathcal{C} \otimes \operatorname{Sp}_{T(n)}
$$

for every $n \in \mathbb{N}$.

Proof. This is a direct consequence of Proposition 5.5.5 and Proposition 5.2.10. 
Corollary 5.5.8. Every $p$-local $\mathcal{C} \in \operatorname{Pr}_{\text {st }}$ is a recollement of $\mathcal{C}_{\leq{ }^{\text {st }} n}$ and $\mathcal{C}_{>^{\text {st }} n}$ for every $n \in \mathbb{N}$.

Proof. Since Sp is a recollement of $L_{n}^{f} \mathrm{Sp}$ and $\left(L_{n}^{f} \mathrm{Sp}\right)^{\perp}=\mathrm{Sp}_{F(n+1)}$, we have by Proposition 5.2.3, that $\mathcal{C} \simeq \mathcal{C} \otimes \mathrm{Sp}$ is a recollement of $\mathcal{C} \otimes L_{n}^{f} \mathrm{Sp}$ and $\mathcal{C} \otimes \mathrm{Sp}_{F(n+1)}$. By Corollary 5.5.7, we get that $\mathcal{C}$ is a recollement of $\mathcal{C}_{\leq{ }^{\text {st }} n}$ and $\mathcal{C}_{>{ }^{\text {st }} n}$.

Let $\mathcal{C}$ be a stable presentable $\infty$-category. For each $n \in \mathbb{N}$, let $\mathcal{C} \stackrel{R_{\leq n}}{\longrightarrow} \mathcal{C}_{\leq{ }^{\mathrm{st}} n}$ be the right adjoint of the inclusion $\mathcal{C}_{\leq \text {st } n} \hookrightarrow \mathcal{C}$ and $\mathcal{C}_{\leq \text {st } n} \stackrel{L_{n}}{\longrightarrow} \mathcal{C}_{n^{\text {st }}}$ the left adjoint of the inclusion $\mathcal{C}_{n^{\text {st }}} \hookrightarrow \mathcal{C}_{\leq^{\text {st }} n}$. We define

$$
P_{n}: \mathcal{C} \stackrel{R_{\leq n}}{\longrightarrow} \mathcal{C}_{\leq{ }^{\text {st }} n} \stackrel{L_{n}}{\longrightarrow} \mathcal{C}_{n^{\text {st }}} .
$$

Proposition 5.5.9. For every p-local $\mathcal{C} \in \operatorname{Pr}_{\mathrm{st}}$ and $X \in \mathcal{C}$. If $P_{n}(X)=0$ for all $n \in \mathbb{N}$, then $X \in \mathcal{C}_{\infty^{\text {st }}}$. In particular, if $\mathcal{C}_{\infty^{\text {st }}}=0$, the collection of functors $P_{n}: \mathcal{C} \rightarrow \mathcal{C}_{n^{\text {st }}}$ is jointly conservative.

Proof. Let $X \in \mathcal{C}$, such that $P_{n}(X)=0$ for all $n$. We shall show by induction that $X \in \mathcal{C}_{>^{\text {st }} n}$ for all $n \in \mathbb{N}$, and hence $X \in \mathcal{C}_{\infty^{\text {st }}}$. Assuming by induction that $X \in \mathcal{C}_{>^{\text {st }} n-1}$ we have

$$
R_{\leq n-1} R_{\leq n}(X)=R_{\leq n-1}(X)=0 .
$$

Therefore $R_{\leq n}(X) \in \mathcal{C}_{>{ }^{\text {st }} n-1}$ and hence

$$
R_{\leq n}(X) \in \mathcal{C}_{\leq{ }^{\text {st }} n} \cap \mathcal{C}_{>{ }^{\text {st }} n-1}=\mathcal{C}_{n^{\text {st }}}
$$

It follows that

$$
P_{n}(X)=L_{n} R_{\leq n}(X)=R_{\leq n}(X) .
$$

Hence, for all $Z \in \mathcal{C}_{\leq{ }^{\text {st }} n}$ we get

$$
\operatorname{Map}(Z, X)=\operatorname{Map}\left(Z, R_{\leq n} X\right)=\operatorname{Map}\left(Z, P_{n} X\right)=\operatorname{Map}(Z, 0)=\mathrm{pt}
$$

and so $X \in \mathcal{C}_{>^{\text {st }} n}$. We can take the base of the induction to be $n=-1$, in which there is nothing to prove.

When further assuming 1-semiadditivity, we get the following:

Proposition 5.5.10. For every $p$-local $\mathcal{C} \in \operatorname{Pr}_{\mathrm{st}}^{\oplus-1}$, we have a canonical equivalence:

$$
\mathcal{C}_{\leq \mathrm{st} n} \simeq \mathcal{C}_{0^{\mathrm{st}}} \times \mathcal{C}_{1^{\mathrm{st}}} \times \cdots \times \mathcal{C}_{n^{\mathrm{st}}}
$$

Proof. By tensoring the equivalence of Theorem 5.4.10 with $\mathcal{C}$, we get an equivalence

$$
\mathcal{C} \otimes \mathrm{CMon}_{1} \otimes L_{n}^{f} \mathrm{Sp} \simeq \mathcal{C} \otimes \prod_{k=0}^{n} \operatorname{Sp}_{T(n)} \simeq \prod_{k=0}^{n}\left(\mathcal{C} \otimes \operatorname{Sp}_{T(n)}\right) .
$$

Since $\mathcal{C}$ is already 1 -semiadditive, $\mathcal{C} \otimes \mathrm{CMon}_{1} \simeq \mathcal{C}$. Thus, the claim follows from Corollary 5.5.7. 


\section{Comparing heights}

For a stable higher semiadditive $\infty$-category, it is natural to compare the stable height with the semiadditive height. First,

Lemma 5.5.11. Let $\mathcal{C} \in \operatorname{Pr}_{\mathrm{st}}^{\oplus-\infty}$ be p-local. For all $n, k \in \mathbb{N} \cup\{\infty\}$, we have $\left(\mathcal{C}_{n^{\mathrm{st}}}\right)_{k} \simeq\left(\mathcal{C}_{k}\right)_{n^{\mathrm{st}}}$.

Proof. Using Theorem 5.3.6 and Proposition 5.5.5 (or Corollary 5.5.6 for $n=\infty$ ) we get

$$
\left(\mathcal{C}_{n^{\mathrm{st}}}\right)_{k} \simeq \mathcal{C} \otimes \operatorname{Sp}_{(p), n^{\mathrm{st}}} \otimes \boldsymbol{\Im}_{k} \simeq\left(\mathcal{C}_{k}\right)_{n^{\mathrm{st}}}
$$

We next observe that for $n \in \mathbb{N}$ the $\infty$-category $\operatorname{Sp}_{(p), n^{\text {st }}}=\operatorname{Sp}_{T(n)}$, which is the mode of stable height $n$, is $\infty$-semiadditive of semiadditive height $n$ (Theorem 4.4.5). Therefore, there is a map of modes $\boldsymbol{\Xi}_{n} \rightarrow \mathrm{Sp}_{T(n)}$, making $\mathrm{Sp}_{T(n)}$ an algebra over $\boldsymbol{\Xi}_{n}$.

Proposition 5.5.12. Let $\mathcal{C} \in \operatorname{Pr}_{\mathrm{st}}^{\oplus-\infty}$ be p-local. For all $n \in \mathbb{N}$ and $k \in \mathbb{N} \cup\{\infty\}$, we have

$$
\left(\mathcal{C}_{n^{\mathrm{st}}}\right)_{k} \simeq\left(\mathcal{C}_{k}\right)_{n^{\mathrm{st}}} \simeq \begin{cases}\mathcal{C}_{n^{\mathrm{st}}} & k=n \\ 0 & k \neq n\end{cases}
$$

Proof. On the one hand, for $k \neq n$ we have $\boldsymbol{צ}_{n} \otimes \boldsymbol{צ}_{k}=0$ (Proposition 5.3.8) and so $\mathrm{Sp}_{T(n)} \otimes \boldsymbol{\Xi}_{k}=0$. On the other, $\operatorname{Sp}_{T(n)} \otimes \boldsymbol{צ}_{n} \simeq \operatorname{Sp}_{T(n)}$ as $\operatorname{Sp}_{T(n)}$ is a $\boldsymbol{\Xi}_{n}$-module. Tensoring these with $\mathcal{C}$ yields the claim.

Given $\mathcal{C} \in \operatorname{Pr}_{\mathrm{st}}^{\oplus-\infty}$, tensoring the map of modes $\boldsymbol{צ}_{n} \rightarrow \operatorname{Sp}_{T(n)}$ with $\mathcal{C}$, yields a map $\mathcal{C}_{n} \rightarrow \mathcal{C}_{n^{\text {st }}}$.

Proposition 5.5.13. Let $\mathcal{C} \in \operatorname{Pr}_{\mathrm{st}}^{\oplus-\infty}$ be p-local. For every $n \in \mathbb{N}$, the map $\mathcal{C}_{n} \rightarrow \mathcal{C}_{n^{\text {st }}}$ admits a fully faithful right adjoint $\mathcal{C}_{n^{\text {st }}} \hookrightarrow \mathcal{C}_{n}$, which exhibits $\mathcal{C}_{n}$ as a recollement of $\mathcal{C}_{n^{\text {st }}}$ and $\left(\mathcal{C}_{n}\right)_{\infty^{\text {st }}}\left(=\left(\mathcal{C}_{\infty^{\text {st }}}\right)_{n}\right)$. In particular, if $\mathcal{C}_{\infty^{\text {st }}}=0$, then $\mathcal{C}_{n} \simeq \mathcal{C}_{n^{\text {st }}}$.

Proof. By Corollary 5.5.8, for every $N \geq n$, the $\infty$-category $\mathcal{C}_{n}$ is a recollement of $\left(\mathcal{C}_{n}\right)_{\leq \text {st } N}$ and $\left(\mathcal{C}_{n}\right)_{>\text {st } N}$. Applying Proposition 5.5.10 and Proposition 5.5.12 to $\mathcal{C}_{n}$ we obtain

$$
\left(\mathcal{C}_{n}\right)_{\leq^{\text {st }} N} \simeq\left(\mathcal{C}_{n}\right)_{0^{\text {st }}} \times\left(\mathcal{C}_{n}\right)_{1^{\text {st }}} \times \cdots \times\left(\mathcal{C}_{n}\right)_{N^{\text {st }}} \simeq \mathcal{C}_{n^{\text {st }}}
$$

Consequently,

$$
\left(\mathcal{C}_{n}\right)_{>\text {st } N}=\left(\mathcal{C}_{n}\right)_{>\text {st } N+1}=\left(\mathcal{C}_{n}\right)_{>\text {st } N+2}=\cdots=\left(\mathcal{C}_{n}\right)_{\infty^{\text {st }}}
$$

and hence, $\mathcal{C}_{n}$ as a recollement of $\mathcal{C}_{n^{\text {st }}}=\left(\mathcal{C}_{n}\right)_{\leq{ }^{\text {st }} N}$ and $\left(\mathcal{C}_{n}\right)_{\infty^{\text {st }}}=\left(\mathcal{C}_{n}\right)_{>\text {st } N}$.

As a consequence, we get a tight connection between $\operatorname{Sp}_{T(n)}$ and $\boldsymbol{צ}_{n}$.

Corollary 5.5.14. The map of modes $\mathbf{\Xi}_{n} \rightarrow \mathrm{Sp}_{T(n)}$ is a smashing localization.

Proof. This follows from Proposition 5.2.15 and Proposition 5.5.13. 


\section{Bootstrap of Semiadditivity}

Based on the classification of higher semiadditive localizations of Sp with respect to homotopy rings, the authors proposed in [CSY18, Conjecture 1.1.5] the "Bootstrap Conjecture", stating that if a presentable stable $p$-local $\infty$-category is 1 -semiadditive, then it is automatically $\infty$-semiadditive. Using the 1-semiadditive decomposition of Theorem 5.4.10 we now provide some partial results in the direction of proving this conjecture. First, given a $p$-local $\mathcal{C} \in \operatorname{Pr}_{\text {st }}$ and $n \in \mathbb{N}$, the $\infty$-category $\mathcal{C}_{n^{\text {st }}}$ is a module over $\operatorname{Sp}_{T(n)}$ and hence over $\boldsymbol{צ}_{n}$. It follows that $\mathcal{C}_{n^{\text {st }}}$ is $\infty$-semiadditive and of height $n$. More generally, if $\mathcal{C}$ is 1 -semiadditive, then by Proposition 5.5.10, we have $\mathcal{C}_{\leq \text {st }_{n}} \simeq \prod_{k=0}^{n} \mathcal{C}_{k^{\text {st }}}$. From this one can deduce that, if $\mathcal{C}$ is 1-semiadditive and every object of $\mathcal{C}$ is of bounded stable height, then $\mathcal{C}$ is $\infty$-semiadditive. However, we shall show that having such a bound on the stable height of the objects of $\mathcal{C}$ is an unnecessarily strong restriction, and it in fact suffices to assume merely that $\mathcal{C}_{\infty^{\text {st }}}=0$.

Since every stable presentable $\infty$-category of stable height exactly $n$ (for some $n$ ) is $\infty$-semiadditive, it has an action of $\pi_{0} \boldsymbol{\Psi}^{[1]}$, and a fortiori of the subring $\mathcal{R}_{1} \subseteq \pi_{0} \boldsymbol{\mho}^{[1]}$ (see Definition 5.4.2). We begin with a generalization of Proposition 5.4.8.

Proposition 5.5.15. For every $n$, there exists $a \in \mathcal{R}_{1}$, such that every $\mathcal{C} \in \operatorname{Pr}_{\mathrm{st}}^{\oplus-1}$ of stable height $k$ is a-complete if $k \leq n$ and a-divisible if $k>n$.

Proof. By Proposition 5.4.8, there exists $a \in \mathcal{R}_{1}$, such that $\operatorname{Sp}_{T(k)}$ is $a$-complete if $k \leq n$ and $a$-divisible if $k>n$. Since $\mathcal{C}$ is an $\operatorname{Sp}_{T(k)}$-module, the action of $a \in \mathcal{R}_{1}$ on $\mathcal{C}$ is via its image in $\pi_{0} \mathbb{S}_{T(k)}$. Thus, it suffices to show that for every $a \in \pi_{0} \mathbb{S}_{T(k)}$, if $\mathrm{Sp}_{T(k)}$ is $a$-complete (resp. $a$-divisible), then $\mathcal{C}$ is also $a$-complete (resp. $a$-divisible). We observe that $\operatorname{Sp}_{T(k)}$ is $a$-divisible if and only if $a$ is invertible, in which case $\mathcal{C}$ is $a$-divisible as well. On the other hand, if $\operatorname{Sp}_{T(k)}$ is $a$-complete, then tensoring with $A:=\mathbb{S}_{T(k)} / a$ is conservative on $\mathrm{Sp}_{T(k)}$. The object $A$ is dualizable with dual $A^{\vee} \simeq \Sigma^{-1} A$ and so tensoring with $A^{\vee}$ is also conservative. Finally, to show that $\mathcal{C}$ is $a$-complete, it suffices to show that tensoring with $A$ is conservative on $\mathcal{C}$, via the left tensoring of $\mathcal{C}$ over $\mathrm{Sp}_{T(k)}$. For every $X, Y \in \mathcal{C}$, we have isomorphisms

$$
\operatorname{hom}_{\mathcal{C}}^{\mathrm{Sp}_{T(k)}}(A \otimes X, Y) \simeq \operatorname{hom}_{\operatorname{Sp}_{T(k)}}^{\mathrm{Sp}_{T(k)}}\left(A, \operatorname{hom}_{\mathcal{C}}^{\mathrm{Sp}_{T(k)}}(X, Y)\right) \simeq A^{\vee} \otimes \operatorname{hom}_{\mathcal{C}} \mathrm{Sp}_{T(k)}(X, Y) .
$$

Hence, if $A \otimes X \simeq 0$, then by the conservativity of $A^{\vee} \otimes(-)$, we get that $\operatorname{hom}_{\mathcal{C}}^{\operatorname{Sp}_{T(k)}}(X, Y)=0$ for all $Y$ and hence $X=0$.

From this we derive a strengthening of Corollary 5.4.9.

Proposition 5.5.16. Let $\mathcal{C}, \mathcal{D} \in \operatorname{Pr}_{\mathrm{st}}^{\oplus-1}$ be p-local. If $\mathrm{Ht}_{\mathrm{st}}(\mathcal{D}) \leq n, \mathrm{Ht}_{\mathrm{st}}(\mathcal{C})>n$ and $\mathcal{C}_{\infty^{\mathrm{st}}}=0$, then every 1-semidditive functor $F: \mathcal{C} \rightarrow \mathcal{D}$ is zero.

Proof. We shall construct an element $a \in \mathcal{R}_{1}$ such that $\mathcal{C}$ is $a$-divisible and $\mathcal{D}$ is $a$-complete. Since $F$ is 1-semiadditive, we shall get that it takes every $X \in \mathcal{C}$ to an object of $\mathcal{D}$ which is both $a$-complete and $a$-divisible (Proposition 5.4.3). This will imply that $F(X)=0$ for all $X \in \mathcal{C}$ and hence that $F$ is zero. By Proposition 5.5.15, there is an $a \in \mathcal{R}_{1}$ such that $\mathcal{C}_{k}$ is $a$-divisible for $k>n$ and $\mathcal{D}_{k}$ is $a$-complete for $k \leq n$. By Proposition 5.5.10, we have

$$
\mathcal{D}=\mathcal{D}_{\leq{ }^{\mathrm{st}} n} \simeq \prod_{0 \leq k \leq n} \mathcal{D}_{k^{\mathrm{st}}}
$$


and hence $\mathcal{D}$ itself is $a$-complete. As for $\mathcal{C}$, by Proposition 5.5.9, we have a jointly conservative collection of functors $P_{n}: \mathcal{C} \rightarrow \mathcal{C}_{n^{\text {st }}}$ for $n \in \mathbb{N}$. Moreover, all the $P_{n^{-S}}$ are 1-semiadditive, as a composition of a left and a right adjoint, and thus $\mathcal{C}$ is $a$-divisible as well.

As a corollary, we get the following partial result in the direction of [CSY18, Conjecture 1.1.5]:

Theorem 5.5.17. Let $\mathcal{C} \in \operatorname{Pr}_{\text {st }}$ be p-local, such that $\mathcal{C}_{\infty^{\text {st }}}=0$. If $\mathcal{C}$ is 1-semiadditive, then $\mathcal{C}$ is $\infty$-semiadditive. Moreover, in this case $\mathcal{C}_{n^{\text {st }}}=\mathcal{C}_{n}$ for all $0 \leq n \leq \infty$ and there is a canonical decomposition

$$
\mathcal{C} \simeq \prod_{n \in \mathbb{N}} \mathcal{C}_{n^{\mathrm{st}}} \simeq \prod_{n \in \mathbb{N}} \mathcal{C}_{n}
$$

Proof. By Corollary 5.5.8, $\mathcal{C}$ is a recollement of $\mathcal{C}_{\leq^{\text {st }} n}$ and $\mathcal{C}_{>}{ }^{\text {st }} n$. Since $\mathcal{C}_{>{ }^{\text {st }} n}$ is 1 -semiadditive and

$$
\left(\mathcal{C}_{>\text {st }} n\right)_{\infty^{\text {st }}} \simeq \mathcal{C}_{\infty^{\text {st }}}=0
$$

every 1-semiadditive functor $F: \mathcal{C}_{>^{\text {st }} n} \rightarrow \mathcal{C}_{<\text {st }}$ is zero by Proposition 5.5.16. It follows that $\mathcal{C}$ is a split recollement of $\mathcal{C}_{\leq{ }^{\text {st }} n}$ and $\mathcal{C}_{>\text {st }}$, and hence a recollement of $\mathcal{C}_{>{ }^{\text {st }} n}$ and $\mathcal{C}_{\leq{ }^{\text {st }} n}$ (i.e. we may switch the roles). By Corollary 4.1.10, we get

$$
\mathcal{C} \simeq \lim _{n \in \mathbb{N}}\left(\mathcal{C}_{\leq{ }^{\text {st }} n}\right) \simeq \lim _{n \in \mathbb{N}}\left(\prod_{k \leq n} \mathcal{C}_{k^{\mathrm{st}}}\right) \simeq \prod_{n \in \mathbb{N}} \mathcal{C}_{n^{\mathrm{st}}}
$$

For every $n \in \mathbb{N}$, the $\infty$-category $\mathcal{C}_{n^{\text {st }}}$ is $\infty$-semiadditive of semiadditive height $n$, hence $\mathcal{C}$ itself is $\infty$-semiadditive and for every $0 \leq k \leq \infty$ we have (Proposition 5.5.12)

$$
\mathcal{C}_{k} \simeq\left(\prod_{n \in \mathbb{N}} \mathcal{C}_{n^{\mathrm{st}}}\right)_{k} \simeq \prod_{n \in \mathbb{N}}\left(\mathcal{C}_{n^{\mathrm{st}}}\right)_{k} \simeq \mathcal{C}_{k^{\mathrm{st}}}
$$

\section{References}

[AKQ19] Gabriel Angelini-Knoll and JD Quigley. Chromatic complexity of the algebraic K-theory of $y(n)$. arXiv preprint arXiv:1908.09164, 2019.

[AR08] Christian Ausoni and John Rognes. The chromatic red-shift in algebraic K-theory. L'Enseignement Mathématique, 54(2):13-15, 2008.

[Bae] John C. Baez. Euler characteristic versus homotopy cardinality. http://math.ucr.edu/home/baez/cardinality/cardinality.pdf.

[Bal16] Paul Balmer. Separable extensions in tensor-triangular geometry and generalized Quillen stratification. Ann. Sci. Éc. Norm. Supér.(4), 49(4):907-925, 2016.

[BG16] Clark Barwick and Saul Glasman. A note on stable recollements. arXiv preprint arXiv:1607.02064, 2016. 
[BG18] Irina Bobkova and Paul G. Goerss. Topological resolutions in K(2)-local homotopy theory at the prime 2. Journal of Topology, 11(4):917-956, 2018.

[BGH17] Agnes Beaudry, Paul G Goerss, and Hans-Werner Henn. Chromatic splitting for the $K(2)$-local sphere at $p=2$. arXiv preprint arXiv:1712.08182, 2017.

[BK72] AK Bousfield and DM Kan. The core of a ring. Journal of Pure and Applied Algebra, 2(1):73-81, 1972.

[BNT18] Ulrich Bunke, Thomas Nikolaus, and Georg Tamme. The Beilinson regulator is a map of ring spectra. Advances in Mathematics, 333:41-86, 2018.

[Car84] Gunnar Carlsson. Equivariant stable homotopy and segal's burnside ring conjecture. Annals of Mathematics, pages 189-224, 1984.

[CM17] Dustin Clausen and Akhil Mathew. A short proof of telescopic Tate vanishing. Proceedings of the American Mathematical Society, 145(12):5413-5417, 2017.

[CSY18] Shachar Carmeli, Tomer M Schlank, and Lior Yanovski. Ambidexterity in chromatic homotopy theory. arXiv preprint arXiv:1811.0205\%, 2018.

[GGN16] David Gepner, Moritz Groth, and Thomas Nikolaus. Universality of multiplicative infinite loop space machines. Algebraic \& Geometric Topology, 15(6):3107-3153, 2016.

[GH15] David Gepner and Rune Haugseng. Enriched $\infty$-categories via non-symmetric $\infty$-operads. Advances in mathematics, 279:575-716, 2015.

[GS96] John P.C. Greenlees and Hal Sadofsky. The Tate spectrum of $v_{n}$-periodic complex oriented theories. Mathematische Zeitschrift, 222(3):391-405, 1996.

[Har17] Yonatan Harpaz. Ambidexterity and the universality of finite spans. arXiv preprint arXiv:1703.09764, 2017.

[Hin16] Vladimir Hinich. Dwyer-Kan localization revisited. Homology Homotopy Appl., 18(1):2748, 2016.

[HL13] Michael Hopkins and Jacob Lurie. Ambidexterity in $K(n)$-local stable homotopy theory. http://www.math.harvard.edu/lurie/, 2013.

[HS96] Mark Hovey and Hal Sadofsky. Tate cohomology lowers chromatic Bousfield classes. Proceedings of the American Mathematical Society, 124(11):3579-3585, 1996.

[HS98] Michael J. Hopkins and Jeffrey H. Smith. Nilpotence and stable homotopy theory II. Ann. of Math. (2), 148(1):1-49, 1998.

[HSSS18] Marc Hoyois, Pavel Safronov, Sarah Scherotzke, and Nicolò Sibilla. The categorified Grothendieck-Riemann-Roch theorem. arXiv preprint arXiv:1804.00879, 2018.

[HY17] Asaf Horev and Lior Yanovski. On conjugates and adjoint descent. Topology and its Applications, 232:140-154, 2017.

[Kuh04] Nicholas J. Kuhn. Tate cohomology and periodic localization of polynomial functors. Inventiones mathematicae, 157(2):345-370, 2004. 
[Lura] Jacob Lurie. Higher algebra. http://www.math.harvard.edu/ lurie/.

[Lurb] Jacob Lurie. Representation theory in intermediate characteristic. http://www.claymath.org/downloads/utah/Notes/Lurie2.pdf.

[Lur09] Jacob Lurie. Higher topos theory, volume 170 of Annals of Mathematics Studies. Princeton University Press, Princeton, NJ, 2009.

[Lur19] Jacob Lurie. Elliptic cohomology III: Tempered cohomology. Available from the author's webpage, 2019.

[MNN17] Akhil Mathew, Niko Naumann, and Justin Noel. Nilpotence and descent in equivariant stable homotopy theory. Advances in Mathematics, 305:994-1084, 2017.

[PS17] Matan Prasma and Tomer M Schlank. Sylow theorems for $\infty$-groups. Topology and its Applications, 222:121-138, 2017. 